\title{
Structure of the space of $G L_{4}\left(\mathbb{Z}_{2}\right)$-coinvariants $\mathbb{Z}_{2} \otimes_{G L_{4}\left(\mathbb{Z}_{2}\right)} P_{A} H_{*}\left(\mathbb{Z}_{2}^{4}, \mathbb{Z}_{2}\right)$ in some generic degrees and its application
}

\author{
ĐĂNG VÕ PHÚC \\ Faculty of Education Studies, University of Khanh Hoa, Vietnam \\ (With profound regards, this work is dedicated to Professors Daciberg Gonçalves and William Singer in \\ commemoration of the special occasion of their birthdays)
}

\begin{abstract}
Fix $k=\mathbb{Z}_{2}$ to be a field of characteristic 2, let $A$ denote the Steenrod algebra over $k$. A problem of immense difficulty in algebraic topology is the determination of a minimal set of $A$-generators for the polynomial ring $P_{q}=k\left[x_{1}, \ldots, x_{q}\right]=H^{*}\left(k^{q}, k\right)$ on $q$ generators $x_{1}, \ldots, x_{q}$ with $\left|x_{1}\right|=\left|x_{2}\right|=$ $\cdots=\left|x_{q}\right|=1$. By way of equivalence, one may choose to write an explicit basis for the cohit space $Q^{q}:=k \otimes_{A} P_{q}$ in each non-negative degree $n$. This subject, which has now a long history, is the content of the classical "hit problem" proposed in [Abstracts Papers Presented Am. Math. Soc. 833 (1987), 55-89]. Furthermore, it is closely related to the $q$-th transfer homomorphism $\operatorname{Tr}_{q}^{A}$ constructed by William Singer in [Math. Z. 202 (1989), 493-523]. This map $\operatorname{Tr}_{q}^{A}$ passes from the space of $G(q)$-coinvariant $k \otimes_{G(q)} P_{A}\left(\left(P_{q}\right)_{n}^{*}\right)$ of $\boldsymbol{Q}^{q}$ to the $k$-cohomology group of the Steenrod algebra, $\operatorname{Ext}_{A}^{q, q+n}(k, k)$, wherein $G(q)$ stands for the general linear group of degree $q$ over the field $k$, and $P_{A}\left(\left(P_{q}\right)_{n}^{*}\right)$ is the primitive part of $\left(P_{q}\right)_{n}^{*}$ under the action of $A$. Particularly, the assertion that $\operatorname{Tr}_{q}^{A}$ is always an injective map has been conjectured by Singer himself, but as of now, this remains an open problem for all $q \geqslant 4$. Accordingly, the aim of the present study is to deal with the Singer conjecture for rank 4 in certain internal degrees. Specifically, by the usage of the techniques of the hit problem in four variables, we explicitly determine the structure of the coinvariant $k \otimes_{G(4)} P_{A}\left(\left(P_{4}\right)_{n}^{*}\right)$ in some generic degrees $n$. Then, applying these results and a representation of $\operatorname{Tr}_{4}^{A}$ via the lambda algebra, we state that Singer's conjecture is true for rank $q=4$ in respective degrees $n$. This has significantly contributed towards the ultimate proof of Singer's conjecture within the rank 4 case.
\end{abstract}

Keywords:

Steenrod algebra; Peterson hit problem; Algebraic transfer; Steenrod squares; Invariant theory

2010 MSC: Primary 55S10; Secondary 55S05, 55T15.

\section{Contents}

1 Introduction $\quad 2$

2 Outline of main results 4

2.1 Degree $n=2^{s+t+1}+2^{s+1}-3, t \geqslant 1, t \neq 3 \ldots \ldots \ldots$

2.2 Degree $n=2^{s+t}+2^{s}-2, t \geqslant 1, t \neq 2,3,4 \ldots \ldots \ldots$

3 Proofs of main results $\quad 12$

3.1 Proof of Theorem $2.1 .1 \ldots \ldots \ldots \ldots \ldots \ldots$

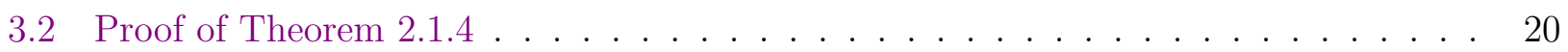

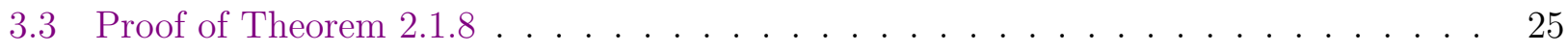

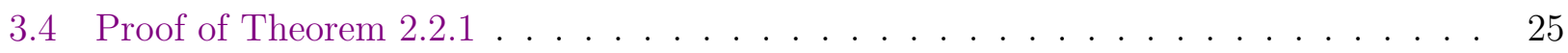

3.5 Proof of Theorem $2.2 .3 \ldots \ldots \ldots \ldots \ldots \ldots$

Acknowledgments. All the results presented in this paper have undergone extensive revisions and refinements since their initial research conducted at the end of 2017, resulting in their current state of perfection. This work was accomplished within a span of five years. I am deeply grateful

Email address: dangphuc150488@gmail.com (ĐẶNG VÕ PHÚC) 
to the editor and anonymous reviewer for their thorough examination of my manuscript. Their insightful comments and suggestions have greatly enhanced the readability and overall quality of this article.

\section{Introduction}

The letter $k$ will be used to represent the prime order field $\mathbb{Z}_{2}$. Let us write $A$ for the classical, singly-graded Steenrod algebra over $k$. It is well-known that $A$ is the ring of stable operations on cohomology with $k$ coefficients, generated by symbols $S q^{t}$, for $t \geqslant 0$, subject to Adem relations. These $S q^{t}$ are called "squaring" operations, since if $\operatorname{deg}(u)=t$, then $S q^{t}(u)=u^{2}$. Let $P_{q}=$ $k\left[x_{1}, \ldots, x_{q}\right]$ denote the polynomial ring generated by $x_{1}, \ldots, x_{q}$, wherein each generator $x_{i}$ has a degree of 1 . This ring may be interpreted as the symmetric power algebra of $\left(k^{q}\right)^{*}$ or as an unstable left $A$-module. Here, $k^{q}$ represents a rank $q$ elementary abelian 2-group which can be viewed as a $q$-dimensional $k$-vector space. The algebra $A$ acts on $P_{q}$ through the Cartan formula $S q^{t}(f g)=\sum_{i+j=t} S q^{i}(f) S q^{j}(g)$ for any $f, g \in P_{q}$ and the rule that $S q^{t}\left(x_{j}\right)=x_{j}$ if $t=0, x_{j}^{2}$ if $t=1$, and 0 otherwise. In addition, the algebra $P_{q}$ is equipped with the usual structure of a right module over the general linear group $G(q):=G L_{q}(k)$ by means of substitution of variables as follows: For each $\sigma=\left(\sigma_{i, j}\right) \in G(q)$ and any $f\left(x_{1}, \ldots, x_{q}\right):=f \in P_{q}$, one defines

$$
(f \sigma)\left(x_{1}, \ldots, x_{q}\right)=f\left(x_{1} \sigma, \ldots, x_{q} \sigma\right)=f\left(\sum_{1 \leqslant i \leqslant q} x_{i} \sigma_{i, 1}, \ldots, \sum_{1 \leqslant i \leqslant q} x_{i} \sigma_{i, q}\right) .
$$

Here and subsequently, we denote by $Q^{q}:=k \otimes_{A} P_{q}$. It is an evident fact that the mapping $k \longrightarrow \operatorname{End}\left(\boldsymbol{Q}^{q}\right)$ is a ring homomorphism, in which $\operatorname{End}\left(\boldsymbol{Q}^{q}\right)$ is the ring of endomorphism of $\boldsymbol{Q}^{q}$ (as abelian group). So, $Q^{q}$ has the structure of a $k$-module (or, equivalently, a $k$-vector space). Let $\left(P_{q}\right)_{n}=H^{n}\left(k^{q}, k\right)$ be the $k$-subspace of $P_{q}$ generated by the homogeneous polynomials of the non-negative degree $n$ in $P_{q}$. (Then $P_{q}=\left\{\left(P_{q}\right)_{n}\right\}_{n \geqslant 0}$.) It was shown that $\left(P_{q}\right)_{n}$ is not a left $A$ submodule of $P_{q}$, but it is a right $G(q)$-module for every $n$. We write $\left(P_{q}\right)^{*}=\left\{\left(P_{q}\right)_{n}^{*}\right\}_{n \geqslant 0}$ for the dual of $P_{q}$, which is also the divided power algebra $\Gamma\left(a_{1}^{(1)}, \ldots, a_{q}^{(1)}\right)$ of $q$ generators $a_{1}^{(1)}, \ldots, a_{q}^{(1)}$, wherein $a_{i}^{(1)}$ is the linear dual to $x_{i}$. We here identify $a_{i}^{(0)}$ with the identity map of $k$. The product on $\Gamma\left(a_{1}^{(1)}, \ldots, a_{q}^{(1)}\right)$ is the Pontrjagin product, that is, for $a_{i}^{(j)}, a_{i}^{(k)}$, if the binary expansions of $j$ and $k$ have no power of two in common, then $a_{i}^{(j)} a_{i}^{(k)}=a_{i}^{(j+k)}$. Otherwise the product is zero. For $i \neq j$, the product of $a_{i}^{(r)}$ and $a_{j}^{(s)}$ is simply $a_{i}^{(r)} a_{j}^{(s)}$. It follows that $\Gamma\left(a_{1}^{(1)}, \ldots, a_{q}^{(1)}\right)$ is generated by the elements $a_{i}^{\left(2^{j}\right)}$ and that for any $k, a_{i}^{(k)}$ is the product of the elements $a_{i}^{\left(2^{j}\right)}$ for each power $2^{j}$ appearing in the binary expansion of $k$. In addition, the algebra $\Gamma\left(a_{1}^{(1)}, \ldots, a_{q}^{(1)}\right)$ supports an operation $u \longmapsto u^{(k)}, k \geqslant 0$ defined for all elements in $\Gamma\left(a_{1}^{(1)}, \ldots, a_{q}^{(1)}\right)$ which do not involve the monomial 1, which satisfies the following rules: For any $u, v \in \Gamma\left(a_{1}^{(1)}, \ldots, a_{q}^{(1)}\right)$,

$$
\begin{aligned}
(u+v)^{(k)} & =\sum_{i+j=k} u^{(i)} v^{(j)} \text { (the divided binomial theorem) and } \\
u^{(i)} u^{(j)} & =\left(\begin{array}{c}
i+j \\
i
\end{array}\right) u^{(i+j)} \text { (the multiplication rule). }
\end{aligned}
$$

This operation is consistent with the notation for $a_{i}^{(k)}$ given above. The right $A$-module structure of this algebra is described by

$$
\left(a_{i}^{(n)}\right) S q^{t}=\left(\begin{array}{c}
n-t \\
t
\end{array}\right) a_{i}^{(n-t)}
$$

together with the Cartan formula. Let us denote by $\boldsymbol{Q}_{n}^{q}$ the $k$-vector subspace of $\boldsymbol{Q}^{q}$ consisting of all the classes represented by the elements in $\left(P_{q}\right)_{n}$. The action of $G(q)$ commutes with that of the Steenrod squares $S q^{t}$ on $P_{q}$, and therefore, there exists an action of $G(q)$ on $\boldsymbol{Q}_{n}^{q}$.

Going back to the Steenrod algebra and its application, a well-known related open problem, as is known, is to determine the set of homotopy classes $\left[\mathbb{S}^{n+q}, \mathbb{S}^{n}\right]$ of continuous based map between spheres. For $n+q>0$, these sets have a natural group structure, and they are abelian when $n+q>1$. The Freudenthal suspension theorem in [Fr38] showed a relationship between the groups $\left[\mathbb{S}^{n+q}, \mathbb{S}^{n}\right]$ for fixed $q$ and varying $n$. The supension map induces a sequence:

$$
\cdots \longrightarrow\left[\mathbb{S}^{n-1+q}, \mathbb{S}^{n-1}\right] \longrightarrow\left[\mathbb{S}^{n+q}, \mathbb{S}^{n}\right] \longrightarrow\left[\mathbb{S}^{n+1+q}, \mathbb{S}^{n+1}\right] \longrightarrow \cdots
$$


of group homomorphisms, and when $n+q>1$, these homomorphisms are isomorphisms. Then, the stable value $\left[\mathbb{S}^{n+q}, \mathbb{S}^{n}\right]$ for $n$ sufficiently large is known as the $q$-th stable homotopy group of spheres, $\pi_{q}$. The cohomology of $A$ with $k$-coefficients, $\operatorname{Ext}_{A}(k, k)=\left\{\operatorname{Ext}_{A}^{q, t}(k, k)\right\}_{q \geqslant 0, t \geqslant 0}$ features prominently in homotopy theory as the $E_{2}$-term of the Adams (bigraded) spectral sequence (the Adams SS, for short), $E_{2}^{q, t}=\operatorname{Ext}_{A}^{q, t}(k, k)$ for the computation of groups $\pi_{q}$. The graded algebra $\operatorname{Ext}_{A}(k, k)$ has attracted the attention of numerous distinguished mathematicians, such as Adams [Ad60], Adem [Ad52], Wall [Wa60], Wang [Wa67], Lin [Li08], Chen [Ch11]. Their efforts have been directed towards unraveling the complex structure of this algebra. Nonetheless, its true nature remains enigmatic up to the present time. The algebraic transfer, also known as the cohomological transfer, as defined by Singer [Si89], is anticipated to be an invaluable instrument in the exploration of Ext groups. This transfer is a $k$-linear map

$$
\operatorname{Tr}_{q}^{A}: k \otimes_{G(q)} P_{A}\left(\left(P_{q}\right)_{n}^{*}\right) \longrightarrow \operatorname{Ext}_{A}^{\operatorname{dim} k^{q}, \operatorname{dim} k^{q}+n}(k, k)=\operatorname{Ext}_{A}^{q, q+n}(k, k),
$$

on which $P_{A}\left(\left(P_{q}\right)_{n}^{*}\right):=\left\langle\left\{\theta \in\left(P_{q}\right)_{n}^{*}:(\theta) S q^{i}=0\right.\right.$, for all $\left.\left.i>0\right\}\right\rangle=\left(\boldsymbol{Q}_{n}^{q}\right)^{*}$ denotes the space of primitive homology classes as a representation of $G(q)$ for all $n$, and the coinvariant $k \otimes_{G(q)} P_{A}\left(\left(P_{q}\right)_{n}^{*}\right)$ is isomorphic as an $k$-vector space to $\left(\boldsymbol{Q}_{n}^{q}\right)^{G(q)}$, the subspace of $G(q)$-invariants of $\boldsymbol{Q}_{n}^{q}$. Singer's transfer has been studied by many topologists like Boardman [Bo93], Bruner et al. [BHH05], Chơn and Hà [CH11, CH12], Hà [Ha07], Hưng [Hu05], Hưng and Quỳnh [HQ09], Minami [Mi95], Nam [Na08], the present writer [Ph20, Ph21a, Ph21b, Ph21c, Ph22a], etc. However, it is also not a straightforward task to explicitly understand the structure of the (co)domain of the transfer. It is worthwhile mentioning that in mostly all of the decade 1980s, Singer believed that the transfer is always an isomorphism. Notwithstanding, with regard to the rank 5 case, he himself asserted in [Si89] that it is not an epimorphism, citing his proof that the indecomposable element $P h_{1} \in$ $\operatorname{Ext}_{A}^{5,14}(k, k)$ does not belong to the image of $\operatorname{Tr}_{5}^{A}$. Subsequently, he put forth the following open conjecture.

Conjecture 1.1 (see [Si89]). $\operatorname{Tr}_{q}^{A}$ is a monomorphism for any $q$.

It has been verified by Singer himself [Si89] for $q=1,2$ and by Boardman [Bo93] for $q=3$. (In fact, the transfer $\operatorname{Tr}_{q}^{A}$ is an isomorphism in those three cases.) These classical results demonstrate the non-trivial nature of the algebraic transfer. Motivated entirely by the already described achievements, we will be concerned with the conjecture of rank $q=4$.

Besides Singer's transfer, the (mod two) lambda algebra ${ }^{1} \Lambda\left[\mathrm{BCK}^{+} 66\right]$ can also be used to describe Ext groups. According to [Wa67], there is a unique differential algebra endomorphism $\theta: \Lambda \longrightarrow \Lambda$ with $\theta\left(\lambda_{n}\right)=\lambda_{2 n+1}$. This one-to-one mapping induces the well-known endomorphism $S q^{0}$ of $\operatorname{Ext}_{A}(k, k)$. It was shown that this classical $S q^{0}$ commutes with the so-called Kameko $S q^{0}$ through Singer's transfer (see also [Bo93], [Mi95] for discussions therein). We denote by $\Lambda^{q, n}$ the $k$-vector subspace of $\Lambda$ generated by all monomials of length $q$. In [CH11], Chơn and Hà defined a noteworthy linear transformation $\psi_{q}:\left(P_{q}\right)_{n}^{*} \longrightarrow \Lambda^{q, n}$, which is determined as follows: for $q=1$, $\psi_{q}\left(\prod_{1 \leqslant s \leqslant q} a_{s}^{\left(j_{s}\right)}\right)=\lambda_{j_{q}}$. If $q>1$, then $\psi_{q}\left(\prod_{1 \leqslant s \leqslant q} a_{s}^{\left(j_{s}\right)}\right)=\sum_{k \geqslant j_{q}} \psi_{q-1}\left(\left(\prod_{1 \leqslant s \leqslant q-1} a_{s}^{\left(j_{s}\right)}\right) S q^{k-j_{q}}\right) \lambda_{k}$, wherein $\prod_{1 \leqslant s \leqslant q} a_{s}^{\left(j_{s}\right)}$ is any element in $\left(P_{q}\right)_{n}^{*}$. This map can be regarded as the $E_{1}$-level of the Singer transfer, and the authors [CH11] have established a result (Theorem 1.2) that is a dual version of the one previously presented by Hưng [Hu90].

Theorem 1.2. With the notation chosen, if $\zeta \in P_{A}\left(\left(P_{q}\right)_{n}^{*}\right)$, then $\psi_{q}(\zeta)$ is a cycle in $\Lambda$ and is a representative of $\operatorname{Tr}_{q}^{A}([\zeta])$.

\footnotetext{
${ }^{1}$ It is widely recognized that the lambda algebra $\Lambda$ is an associative, differential bigraded algebra, with generators $\lambda_{n} \in \Lambda^{1, n}$ for $n \geqslant 0$, and the Adem relations$$
\lambda_{i} \lambda_{2 i+n+1}=\sum_{j \geqslant 0}\left(\begin{array}{c}
n-j-1 \\
j
\end{array}\right) \lambda_{i+n-j} \lambda_{2 i+1+j},
$$$$
(i \geqslant 0, n \geqslant 0)
$$

with differential

$$
d\left(\lambda_{n-1}\right)=\sum_{j \geqslant 1}\left(\begin{array}{c}
n-j-1 \\
j
\end{array}\right) \lambda_{n-j-1} \lambda_{j-1}, d\left(\lambda_{0}\right)=0 .
$$$$
(n \geqslant 1),
$$

We refer to [Wa67] for the relations (1) and $\left[\mathrm{BCK}^{+} 66, \operatorname{Pr} 70\right]$ for that the differential in (2) is a well-defined endomorphism of $\Lambda$. One can view $\Lambda$ as the $E_{1}$-term of the Adams SS converging to the 2-component of the stable homotopy groups of spheres.
} 
As indicated in the abstract, and to close this introductory section, we aim to explicitly describe the domain and codomain of the Singer algebraic transfe of rank four in some generic degrees. As immediate consequences, we answer Singer's Conjecture 1.1 for $q=4$ in respective degrees. This has made a significant contribution towards the final verification of Singer's conjecture in the case of homological degree 4. Our approach relies on the techniques developed for the "hit" problem for the polynomial algebra $P_{4}[\mathrm{Su} 14, \mathrm{Su} 15]$ and the chain-level representation of the fourth transfer homomorphism $\operatorname{Tr}_{4}^{A}$ via the lambda algebra $\Lambda$. The primary results achieved by us are formulated in the sequel.

\section{Outline of main results}

To motivate the statement of our main results, we review the hit problem in the literature [Pe87] and the structure of $\operatorname{Ext}_{A}^{q, *}(k, k)$ for $q \leqslant 4$. Let us recall that the graded polynomial $\operatorname{ring} P_{q}=\left\{\left(P_{q}\right)_{n}\right\}_{n \geqslant 0}$ is a left $A$-module. It makes sense to ask the question: What is a minimal generating set for $P_{q}$ as an A-module? We say that a polynomial $f \in\left(P_{q}\right)_{n}$ is "hit" (or $A$-decomposable), if it satisfies a hit equation, $f=\sum_{t>0} S q^{t}\left(f_{t}\right)$ for some $S q^{t} \in A$ and $f_{t} \in\left(P_{q}\right)_{n-t}$. Said differently, $f$ belongs to $k G(q)$-submodule, $\widehat{A}\left(P_{q}\right)_{n}:=\left(P_{q}\right)_{n} \cap \widehat{A} P_{q}=\left(P_{q}\right)_{n} \cap \sum_{t>0} \operatorname{Im}\left(S q^{t}\right)$, wherein $\widehat{A}$ is the kernel of the epimorphism of graded $k$-algebras $A \longrightarrow k$ given by $S q^{0} \longmapsto 1$ and $S q^{i} \longmapsto 0$ for all $i>0$. The problem of finding a minimal set of $A$-generators for the ring $P_{q}$, commonly referred to as the "hit problem" whose precise meaning remains elusive, has been extensively studied by prominent algebraic topologists such as Peterson [Pe87], Singer [Si91], and many others since the 1980s. This problem has emerged as an active research direction in recent decades. The earliest work on this subject dates back to 1987, with the seminal contribution of Peterson [Pe87], who established a close connection between the cobordism theory ${ }^{2}$ of manifolds and the hit problem. Quite recently, many classical results with numerous references related to these subjects can be found in the monographs by Walker and Wood [WW18a, WW18b].

It is worth pointing out that in the examination of the hit problem, if $k$ has a trivial action from $A$, then it becomes necessary to find a basis for the "cohit" $k$-module $\boldsymbol{Q}_{n}^{q}=\left(k \otimes_{A} P_{q}\right)_{n}=$ $\left(P_{q}\right)_{n} / \widehat{A}\left(P_{q}\right)_{n}, q \geqslant 1, n \geqslant 1$. This space was fully studied by Peterson [Pe87] for $q=1,2$, by Kameko [Ka90] and Janfada [Ja08] for $q=3$, and by Sum [Su14, Su15] for $q=4$. (We would like to bring to the readers' attention that, to the best of our knowledge, the dimension result for $\boldsymbol{Q}_{n}^{4}$ has, in fact, been announced in the preprint [Ka03] through computer calculations. Later on, inspired by some outcomes in Kameko's thesis [Ka90], with the utilization of the Microsoft Excel Spreadsheet Software, and by developing an inductive formula devised by Nam [Na04] for the dimension of $\boldsymbol{Q}_{n}^{q}$, Sum elaborately established the "monomial" bases for $\boldsymbol{Q}_{n}^{4}$ in his spectacular works [Su14, Su15].) Remarkably, an interesting conjecture made by Kameko [Ka90] that was believed to be true by most topologists at the time is that in degrees $n$, the order of the quotient group of $G(q)$ by the Borel subgroup is always an upper bound for the dimension of $\boldsymbol{Q}_{n}^{q}$. Regrettably, his prediction is generally incorrect. Interested readers can find illustrative counterexamples in [Su10, WW18b]. We suggest that readers refer to recent works, such as [Ph20, Ph21a, Ph21b, Ph23a, Ph22a, Ph22b, Su23], for comprehensive information on the solutions of the hit problem for variables greater than 4 in certain "generic" degrees. Additionally, in [Ph23b], our initial study yielded new results on the symmetric hit problem for the symmetric polynomial algebra in four variables, $P_{4}^{\Sigma_{4}}$. It is interesting to note that Janfada [Ja00] has solved the symmetric hit problem for variables $q<4$, but no prior research has been conducted on the cases wherein $q \geqslant 4$.

The following Kameko maps [Ka90] are frequently used to study the hit problem: For each $n \geqslant 0$, the down Kameko map $\overline{S q}^{0}:\left(P_{q}\right)_{2 n+q} \longrightarrow\left(P_{q}\right)_{n}$ is a surjective linear map defined on monomials by $\overline{S q}^{0}(f)=g$ if $f=\prod_{1 \leqslant i \leqslant q} x_{i} g^{2}$ and $\overline{S q}^{0}(f)=0$ otherwise. This map sends hit polynomials to hit polynomials. The up Kameko map $\varphi:\left(P_{q}\right)_{n} \longrightarrow\left(P_{q}\right)_{2 n+q}$ is an injective linear map defined on monomials by $\varphi(g)=\prod_{1 \leqslant i \leqslant q} x_{i} g^{2}$. In general, this map does not send hit polynomials to hit polynomials. (By way of illustration, we observe that in the $A$-module $P_{7}$, while the monomials $g_{i}=x_{i}^{2^{m-1}-4}$, for $1 \leqslant i \leqslant 7$, and any $m \geqslant 4$, are hit, the monomials $\varphi\left(g_{i}\right)=x_{1} x_{2} \ldots x_{7} g_{i}^{2} \in\left(P_{7}\right)_{2^{m}-1}$

\footnotetext{
${ }^{2}$ The cobordism theory, initially formulated by Thom [Th54] for smooth manifolds (i.e., differentiable), examines the distinctions between closed manifolds and those that are boundaries. At the 1958 International Congress of Mathematicians in Edinburgh, Thom was awarded the Field Medal in recognition of his contributions to the development of cobordism.
} 
remain unhit. This is consistent with the result of Walker and Wood [WW18a], which established that no monomial of degree $2^{m}-1$ in $P_{q}$ is hit.) Nonetheless, by considering the arithmetic function $\mu(n)=\min \{* \in \mathbb{N}: \alpha(n+*) \leqslant *\}$, wherein the $\alpha$ function counts the number of ones in the binary expansion of its argument, it has been demonstrated that if $\mu(2 n+q)=q$, then $\varphi$ sends hit polynomials to hit polynomials and induces a map of $k G(q)$-modules $\varphi: \boldsymbol{Q}_{n}^{q} \longrightarrow \boldsymbol{Q}_{2 n+q}^{q}$ that is the inverse of $\overline{S q}^{0}$. In [Pe87], Peterson conjectured that $Q_{n}^{q}$ is trivial if and only if $\mu(n)>q$. This was finally proved by Wood [Wo89] after being investigated by a number of researchers. (Alternatively, in [Si91], Singer proved another result which generalizes the Peterson conjecture and identifies a new class of monomials in $\widehat{A}\left(P_{q}\right)_{n}$.) Thus, based on these aforementioned facts, it is adequate to resolve the hit problem in degrees $n$ such that $\mu(n)<q$.

The image of the algebraic transfer of rank 4 was thoroughly investigated by Bruner et al. [BHH05], Hưng [Hu05], Hà [Ha07], Nam [Na08], Hưng-Quỳnh [HQ09], Chơn-Hà [CH12]. More precisely, the image of $\operatorname{Tr}_{4}^{A}$ contains all the elements of the $S q^{0}$-families $\left\{d_{s}: s \geqslant 0\right\},\left\{e_{s}: s \geqslant 0\right\}$ (see Hà [Ha07]), $\left\{f_{s}: s \geqslant 0\right\}$ (see Nam [Na08]) and $\left\{p_{s}: s \geqslant 0\right\}$ (see Hưng-Quỳnh [HQ09], ChơnHà [CH12] and discussions therein), but none from the $S q^{0}$-families $\left\{g_{s+1}: s \geqslant 0\right\},\left\{D_{3}(s): s \geqslant 0\right\}$ and $\left\{p_{s}^{\prime}: s \geqslant 0\right\}$ (see Bruner et al. [BHH05], Hưng [Hu05]). Additionally, the elements that are product of the $h_{i}, i \geqslant 0$, in the image of the transfer. (We wish to highlight the fact, in particular, that despite being published more than 10 years ago, Hung-Quynh's paper [HQ09] is limited to a brief communication without proof. In addition, Hung's calculations [Hu05] for the codomain of the fourth transfer in stems of $D_{3}(1)$ and $p_{1}$ were conducted using a computer algebra system. It is therefore considered necessary to reassess these results.)

In order to describe the codomain of $\operatorname{Tr}_{4}^{A}$, we need the well-known result (Theorem 2.1) below, which will subsequently be used in our proofs of main results.

Theorem 2.1 (see [Ad60, Ad52, Wa60, Wa67, Li08]).

- $\operatorname{Ext}_{A}^{1, *}(k, k)$ is generated by $h_{i}$ for $i \geqslant 0$;

- $\operatorname{Ext}_{A}^{2, *}(k, k)$ is generated by $h_{i} h_{j}$ for $j \geqslant i \geqslant 0$ and $j \neq i+1$;

- $\operatorname{Ext}_{A}^{3, *}(k, k)$ is generated by $h_{i} h_{j} h_{\ell}, c_{t}$ for $t \geqslant 0 ; \ell \geqslant j \geqslant i \geqslant 0$, and subject only to the relations $h_{i} h_{i+1}=0, h_{i} h_{i+2}^{2}=0$ and $h_{i}^{3}=h_{i-1}^{2} h_{i+1}$;

- $\operatorname{Ext}_{A}^{4, *}(k, k)$ is generated by $h_{i} h_{j} h_{\ell} h_{m}, h_{u} c_{v}, d_{t}, e_{t}, f_{t}, g_{t+1}, p_{t}, D_{3}(t), p_{t}^{\prime}$ for $m \geqslant \ell \geqslant j \geqslant i \geqslant$ $0, u, v, t \geqslant 0$, and subject to the relations in iii) together with $h_{i}^{2} h_{i+3}^{2}=0, h_{v-1} c_{v}=0, h_{v} c_{v}=$ $0, h_{v+2} c_{v}=0$ and $h_{v+3} c_{v}=0$.

We are ready to formulate the main results of the paper. With the condition $\mu(n)<q=4$, we only consider the following "generic" degrees $n$ :

$$
\begin{aligned}
& \text { i) } \quad n=2^{s+1}-t, t \leqslant 3, \\
& \text { ii) } \quad n=2^{s+t+1}+2^{s+1}-3, \\
& \text { iii) } \quad n=2^{s+t}+2^{s}-2, \\
& \text { iv) } \quad n=2^{s+t+u}+2^{s+t}+2^{s}-3,
\end{aligned}
$$

whenever $s, t, u$ are positive integers. As the domain of the fourth Singer transfer is dual to the invariant $\left(\boldsymbol{Q}_{n}^{4}\right)^{G(4)}$, it suffices to verify Singer's conjecture for $\operatorname{Tr}_{4}^{A}$ in degrees of the form (3). For this purpose, Sum has conducted a study of the conjecture for item $i$ ), as detailed in [Su18, Theorem 4.1]. However, it is worthwhile noting that the proof provided in [Su18] demonstrating the isomorphism of Singer's transfer in bidegrees $(4,61)$ and $(4,126)$ has been found to be incorrect. Concretely,

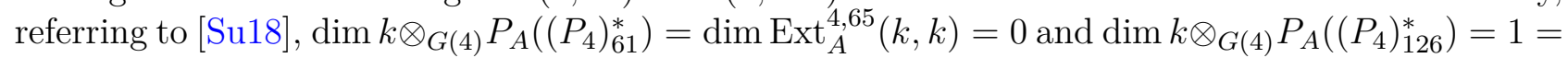
$\operatorname{dim} \operatorname{Ext}_{A}^{4,130}(k, k)$, but by Theorem 2.1, we must have $\operatorname{Ext}_{A}^{4,65}(k, k)=\left\langle D_{3}(0)\right\rangle$ and $\operatorname{Ext}_{A}^{4,130}(k, k)=$ $\left\langle h_{0}^{2} h_{6}^{2}, D_{3}(1)\right\rangle$, wherein $h_{0}^{2} h_{6}^{2} \neq 0, h_{0}^{2} h_{6}^{2} \in \operatorname{Im}\left(\operatorname{Tr}_{4}^{A}\right)$ and $D_{3}(s) \neq 0, s \geqslant 0, D_{3}(s) \notin \operatorname{Im}\left(\operatorname{Tr}_{4}^{A}\right)$ (see Remark 2.2.5). We also direct readers to the corrected version in [Su21a] for further details. In [Ph21c, Ph22a], we have confirmed the conjecture for item iii) with $t=2$, 4 and for item $i v$ ).

The principal objective of this work is to confirm the conjecture for item $i i$ ) with $t \neq 3$ and for item iii) with $t \neq 2,3,4$. Let's examine these in two subsections below. We also make use of the following fact.

Remark 2.2. For each non-negative integer $s$, the monomial $\lambda_{2^{s}-1} \in \Lambda^{1,2^{s}-1}$ is a cycle in the algebra $\Lambda$ and is a representative of the Adams element $h_{s} \in \operatorname{Ext}_{A}^{1,2^{s}}(k, k)$. 
2.1. Degree $n=2^{s+t+1}+2^{s+1}-3, t \geqslant 1, t \neq 3$

Case $\boldsymbol{t}=1$. We have $2^{s+t+1}+2^{s+1}-3=2^{s+2}+2^{s+1}-3$. Based upon an admissible basis of the $k$-vector space $Q_{2^{s+2}+2^{s+1}-3}^{4}$ in [Su14], we find that

Theorem 2.1.1. For a positive integer $s$, then

$$
\operatorname{dim} k \otimes_{G(4)} P_{A}\left(\left(P_{4}\right)_{2^{s+2}+2^{s+1}-3}^{*}\right)= \begin{cases}0 & \text { if } s=2, \\ 1 & \text { if } s \neq 2 .\end{cases}
$$

Let us sketch the proof of the theorem. Firstly, by Sum [Su14], the dimensions of the $k$-vector spaces $\boldsymbol{Q}_{3\left(2^{s}-1\right)+3.2^{s}}^{4}$ are determined as follows:

$$
\operatorname{dim} Q_{3\left(2^{s}-1\right)+3.2^{s}}^{4}= \begin{cases}46 & \text { if } s=1 \\ 94 & \text { if } s=2 \\ 105 & \text { if } s \geqslant 3 .\end{cases}
$$

Thanks to these results, for $s \in\{1,2\}$, a direct computation yields that

$$
k \otimes_{G(4)} P_{A}\left(\left(P_{4}\right)_{2^{s+2}+2^{s+1}-3}^{*}\right)= \begin{cases}\left\langle\left[\zeta_{1}\right]\right\rangle & \text { if } s=1, \\ 0 & \text { if } s=2,\end{cases}
$$

wherein $\zeta_{1}=a_{1}^{(1)} a_{2}^{(3)} a_{3}^{(3)} a_{4}^{(2)}+a_{1}^{(1)} a_{2}^{(3)} a_{3}^{(4)} a_{4}^{(1)}+a_{1}^{(1)} a_{2}^{(5)} a_{3}^{(2)} a_{4}^{(1)}+a_{1}^{(1)} a_{2}^{(6)} a_{3}^{(1)} a_{4}^{(1)} \in P\left(\left(P_{4}\right)_{3\left(2^{1}-1\right)+3.2^{1}}^{*}\right.$. By the unstable condition, to verify that $\zeta_{1}$ is $\widehat{A}$-annihilated, we only need to consider the effects of $S q^{1}$ and $S q^{2}$.

For $s \geqslant 3$, based on a monomial basis of $Q_{3\left(2^{s}-1\right)+3.2^{s}}^{4}$, we derive

$$
\operatorname{dim} k \otimes_{G(4)} P_{A}\left(\left(P_{4}\right)_{2^{s+2}+2^{s+1}-3}^{*}\right) \leqslant 1 .
$$

On the other side, it is pretty clear that the elements $\zeta_{s}=a_{2}^{\left(2^{s+1}-1\right)} a_{3}^{\left(2^{s+1}-1\right)} a_{4}^{\left(2^{s+1}-1\right)}$ in $\left(P_{4}\right)_{3\left(2^{s}-1\right)+3.2^{s}}^{*}$ are $\widehat{A}$-annihilated. Since $\zeta_{s} \in P_{A}\left(\left(P_{4}\right)_{2^{s+2}+2^{s+1}-3}^{*}\right)$, by Remark 2.2 and Theorem 1.2, we must have that the cycles $\psi_{4}\left(\zeta_{s}\right)=\lambda_{0} \lambda_{2^{s+1}-1}^{3}$ in $\Lambda$ are representative of the non-zero elements $h_{0} h_{s+1}^{3} \in$ $\operatorname{Ext}_{A}^{4,6 \cdot 2^{s}+1}(k, k)$. These lead to $h_{0} h_{s+1}^{3} \in \operatorname{Im}\left(\operatorname{Tr}_{4}^{A}\right)$. On the other hand, by Theorem 2.1,

$$
\operatorname{Ext}_{A}^{4,6.2^{s}+1}(k, k)= \begin{cases}\left\langle h_{0} h_{2}^{3}, h_{1} c_{0}\right\rangle=\left\langle h_{1} c_{0}\right\rangle & \text { if } s=1, \\ \left\langle h_{0} h_{s+1}^{3}\right\rangle & \text { if } s \geqslant 2,\end{cases}
$$

wherein $h_{0} h_{s+1}^{3}=0$ for $s=2$ and $h_{0} h_{s+1}^{3}=h_{0} h_{s}^{2} h_{s+2} \neq 0$ for all $s \geqslant 3$. These data together with the inequality (4) imply that the coinvariants space $k \otimes_{G(4)} P_{A}\left(\left(P_{4}\right)_{2^{s+2}+2^{s+1}-3}\right)$ is 1-dimensional. Moreover, by a direct computation using the monomial bases of $\boldsymbol{Q}_{3\left(2^{s}-1\right)+3.2^{s}}^{4}$, one can obtain that the coinvariants $k \otimes_{G(4)} P_{A}\left(\left(P_{4}\right)_{2^{s+2}+2^{s+1}-3}^{*}\right)$ are generated by $\left[\zeta_{s}\right]$, for any $s \geqslant 3$.

Remark 2.1.2. Clearly, $\lambda_{3}^{2} \lambda_{2}$ is a representative of the non-zero element $c_{0} \in \operatorname{Ext}_{A}^{3,11}(k, k)$. Then, since $\zeta_{1}$ is $\widehat{A}$-annihilated, by a direct computation using the representation of $\operatorname{Tr}_{4}^{A}$ over $\Lambda$ and Theorem 1.2, we deduce that

$$
\begin{aligned}
& \psi_{4}\left(a_{1}^{(1)} a_{2}^{(3)} a_{3}^{(3)} a_{4}^{(2)}\right)=\lambda_{1} \lambda_{3}^{2} \lambda_{2}+\lambda_{1} \lambda_{3} \lambda_{4} \lambda_{1}+\lambda_{1} \lambda_{4} \lambda_{3} \lambda_{1}, \\
& \psi_{4}\left(a_{1}^{(1)} a_{2}^{(3)} a_{3}^{(4)} a_{4}^{(1)}\right)=\lambda_{1} \lambda_{3} \lambda_{4} \lambda_{1}+\lambda_{1} \lambda_{4} \lambda_{3} \lambda_{1}+\lambda_{1} \lambda_{5} \lambda_{2} \lambda_{1}, \\
& \psi_{4}\left(a_{1}^{(1)} a_{2}^{(5)} a_{3}^{(2)} a_{4}^{(1)}\right)=\lambda_{1} \lambda_{5} \lambda_{2} \lambda_{1}+\lambda_{1} \lambda_{6} \lambda_{1}^{2}, \\
& \psi_{4}\left(a_{1}^{(1)} a_{2}^{(6)} a_{3}^{(1)} a_{4}^{(1)}\right)=\lambda_{1} \lambda_{6} \lambda_{1}^{2},
\end{aligned}
$$

and therefore the cycle $\psi_{4}\left(\zeta_{1}\right)=\lambda_{1} \lambda_{3}^{2} \lambda_{2}$ in $\Lambda^{4,9}$ is a representative of the element $h_{1} c_{0} \in \operatorname{Ext}_{A}^{4,13}(k, k)$. This fact together with the equality (5) show that the non-zero element $h_{1} c_{0}$ belongs to the image of the fourth algebraic transfer. 
It can be deduced by combining Remark 2.1.2 with Theorem 2.1.1 and the equality (5) that

Corollary 2.1.3. The Singer transfer

$$
\operatorname{Tr}_{4}^{A}: k \otimes_{G(4)} P_{A}\left(\left(P_{4}\right)_{6.2^{s}-3}^{*}\right) \longrightarrow \operatorname{Ext}_{A}^{4,6.2^{s}+1}(k, k)
$$

is an isomorphism for every positive integer $s$.

Case $\boldsymbol{t}=2$. For this, $2^{s+t+1}+2^{s+1}-3=2^{s+3}+2^{s+1}-3$, and the structure of the coinvariant spaces $k \otimes_{G(4)} P_{A}\left(\left(P_{4}\right)_{2^{s+3}+2^{s+1}-3}^{*}\right)$ are given as follows.

Theorem 2.1.4. With a positive integer $s$, we have

$$
k \otimes_{G(4)} P_{A}\left(\left(P_{4}\right)_{2^{s+3}+2^{s+1}-3}^{*}\right)= \begin{cases}\langle[\zeta]\rangle & \text { if } s=1, \\ 0 & \text { if } s>1,\end{cases}
$$

wherein $\zeta$ is the following sum:

$$
\begin{aligned}
& a_{1}^{(5)} a_{2}^{(5)} a_{3}^{(5)} a_{4}^{(2)}+a_{1}^{(5)} a_{2}^{(5)} a_{3}^{(6)} a_{4}^{(1)}+a_{1}^{(3)} a_{2}^{(5)} a_{3}^{(8)} a_{4}^{(1)}+a_{1}^{(5)} a_{2}^{(3)} a_{3}^{(8)} a_{4}^{(1)}+a_{1}^{(3)} a_{2}^{(6)} a_{3}^{(7)} a_{4}^{(1)} \\
& +a_{1}^{(5)} a_{2}^{(7)} a_{3}^{(4)} a_{4}^{(1)}+a_{1}^{(7)} a_{2}^{(5)} a_{3}^{(4)} a_{4}^{(1)}+a_{1}^{(3)} a_{2}^{(9)} a_{3}^{(4)} a_{4}^{(1)}+a_{1}^{(9)} a_{2}^{(3)} a_{3}^{(4)} a_{4}^{(1)}+a_{1}^{(3)} a_{2}^{(9)} a_{3}^{(3)} a_{4}^{(2)} \\
& +a_{1}^{(9)} a_{2}^{(3)} a_{3}^{(3)} a_{4}^{(2)}+a_{1}^{(5)} a_{2}^{(9)} a_{3}^{(2)} a_{4}^{(1)}+a_{1}^{(9)} a_{2}^{(5)} a_{3}^{(2)} a_{4}^{(1)}+a_{1}^{(5)} a_{2}^{(10)} a_{3}^{(1)} a_{4}^{(1)}+a_{1}^{(9)} a_{2}^{(6)} a_{3}^{(1)} a_{4}^{(1)} \\
& +a_{1}^{(3)} a_{2}^{(11)} a_{3}^{(2)} a_{4}^{(1)}+a_{1}^{(11)} a_{2}^{(3)} a_{3}^{(2)} a_{4}^{(1)}+a_{1}^{(5)} a_{2}^{(5)} a_{3}^{(3)} a_{4}^{(4)}+a_{1}^{(5)} a_{2}^{(3)} a_{3}^{(5)} a_{4}^{(4)}+a_{1}^{(3)} a_{2}^{(5)} a_{3}^{(5)} a_{4}^{(4)} \\
& +a_{1}^{(3)} a_{2}^{(12)} a_{3}^{(1)} a_{4}^{(1)}+a_{1}^{(11)} a_{2}^{(4)} a_{3}^{(1)} a_{4}^{(1)}+a_{1}^{(7)} a_{2}^{(8)} a_{3}^{(1)} a_{4}^{(1)}+a_{1}^{(7)} a_{2}^{(7)} a_{3}^{(1)} a_{4}^{(2)}+a_{1}^{(13)} a_{2}^{(2)} a_{3}^{(1)} a_{4}^{(1)} \\
& +a_{1}^{(14)} a_{2}^{(1)} a_{3}^{(1)} a_{4}^{(1)}+a_{1}^{(6)} a_{2}^{(5)} a_{3}^{(3)} a_{4}^{(3)}+a_{1}^{(5)} a_{2}^{(3)} a_{3}^{(6)} a_{4}^{(3)}+a_{1}^{(3)} a_{2}^{(6)} a_{3}^{(5)} a_{4}^{(3)}+a_{1}^{(6)} a_{2}^{(3)} a_{3}^{(3)} a_{4}^{(5)} \\
& +a_{1}^{(3)} a_{2}^{(3)} a_{3}^{(6)} a_{4}^{(5)}+a_{1}^{(3)} a_{2}^{(6)} a_{3}^{(3)} a_{4}^{(5)}+a_{1}^{(5)} a_{2}^{(3)} a_{3}^{(3)} a_{4}^{(6)}+a_{1}^{(3)} a_{2}^{(5)} a_{3}^{(3)} a_{4}^{(6)}+a_{1}^{(3)} a_{2}^{(3)} a_{3}^{(5)} a_{4}^{(6)} \\
& +a_{1}^{(3)} a_{2}^{(3)} a_{3}^{(3)} a_{4}^{(8)}+a_{1}^{(3)} a_{2}^{(3)} a_{3}^{(4)} a_{4}^{(7)}+a_{1}^{(3)} a_{2}^{(5)} a_{3}^{(2)} a_{4}^{(7)}+a_{1}^{(3)} a_{2}^{(6)} a_{3}^{(1)} a_{4}^{(7)}+a_{1}^{(3)} a_{2}^{(3)} a_{3}^{(9)} a_{4}^{(2)} \\
& +a_{1}^{(3)} a_{2}^{(3)} a_{3}^{(10)} a_{4}^{(1)}+a_{1}^{(5)} a_{2}^{(3)} a_{3}^{(7)} a_{4}^{(2)}+a_{1}^{(5)} a_{2}^{(7)} a_{3}^{(3)} a_{4}^{(2)}+a_{1}^{(7)} a_{2}^{(5)} a_{3}^{(3)} a_{4}^{(2)} .
\end{aligned}
$$

Noting that the element $\zeta$ is the same as in [Ha07], and so, it is $\bar{A}$-annihilated. The proof of the theorem is based on the admissible bases of the $k$-vector spaces $Q_{2^{s+3}+2^{s+1}-3}^{4}$ (see [Su14]).

It is apparently that the non-zero element $e_{0}$ in $\operatorname{Ext}_{A}^{4,21}(k, k)$ is represented by the cycle

$$
\bar{e}_{0}:=\lambda_{3}^{3} \lambda_{8}+\lambda_{3} \lambda_{5}^{2} \lambda_{4}+\lambda_{3}^{2} \lambda_{7} \lambda_{4}+\lambda_{7} \lambda_{5} \lambda_{3} \lambda_{2}+\lambda_{3}^{2} \lambda_{5} \lambda_{6}
$$

in $\Lambda^{4,17}$. Then, since $\zeta \in P\left(\left(P_{4}\right)_{2^{1+3}+2^{1+1}-3}^{*}\right)$, by a direct computation using the differential (2) and the representation of $\operatorname{Tr}_{4}^{A}$ over $\Lambda$, we conclude that

$$
\psi_{4}(\zeta)=\bar{e}_{0}+d\left(\lambda_{3} \lambda_{5} \lambda_{10}+\lambda_{3} \lambda_{12} \lambda_{3}+\lambda_{4} \lambda_{7}^{2}+\lambda_{0} \lambda_{11} \lambda_{7}\right)
$$

is a cycle in $\Lambda^{4,2^{1+3}+2^{1+1}-3}$, and so, one derives

$$
\operatorname{Tr}_{4}^{A}([\zeta])=\left[\psi_{4}(\zeta)\right]=\left[\bar{e}_{0}\right]=e_{0} .
$$

On the other side, using Theorem 2.1, we may deduce that

$$
\operatorname{Ext}_{A}^{4,2^{s+3}+2^{s+1}+1}(k, k)= \begin{cases}\left\langle h_{0} h_{2} h_{3}^{2}, e_{0}\right\rangle=\left\langle e_{0}\right\rangle & \text { if } s=1 \\ \left\langle h_{0} h_{s+1} h_{s+2}^{2}\right\rangle=0 & \text { if } s>1\end{cases}
$$

Combining Theorem 2.1.4 with the equalities (6) and (7), we obtain

Corollary 2.1.5. The transfer map $\operatorname{Tr}_{4}^{A}$ is an isomorphism in degree $2^{s+3}+2^{s+1}-3$ for any $s \geqslant 1$.

Remark 2.1.6. Let us consider the up Kameko map $\varphi: P_{4} \longrightarrow P_{4}, u \longmapsto x_{1} \ldots x_{4} u^{2}$. It can be easily seen that for each integer $s \geqslant 1$,

$$
k \otimes_{G(4)} P_{A}\left(\left(P_{4}\right)_{21.2^{s}-4}^{*}\right) \cong k \otimes_{G(4)} P_{A}\left(\left(P_{4}\right)_{38}^{*}\right) .
$$

Combining this with Theorem 2.1.4, Proposition 3.2.1 (see Section three) and a previous result in [Ph21c], we get

$$
k \otimes_{G(4)} P_{A}\left(\left(P_{4}\right)_{21.2^{s}-4}^{*}\right)= \begin{cases}k\left[\zeta_{0}\right]=k[\zeta]=k([\widetilde{\zeta}])^{*} & \text { if } s=0, \\ \left\langle\left[a_{1}^{\left(2^{s-1}-1\right)} a_{2}^{\left(2^{s-1}-1\right)} a_{3}^{\left(2^{s+2}-1\right)} a_{4}^{\left(2^{s+4}-1\right)}\right],\left[\zeta_{s}\right]=\left(\left[\varphi^{s}(\widetilde{\zeta})\right]\right)^{*}\right\rangle & \text { if } s \geqslant 1,\end{cases}
$$


wherein the polynomials $\zeta=\zeta_{0}$ and $\widetilde{\zeta}$ are respectively described as in Theorem 2.1.4 and Proposition 3.2.1, while $\zeta_{s}(s \geqslant 0)$ is the following sum:

$$
\begin{aligned}
& a_{1}^{\left(2^{s+2}+2^{s+1}-1\right)} a_{2}^{\left(2^{s+2}+2^{s+1}-1\right)} a_{3}^{\left(2^{s+2}+2^{s+1}-1\right)} a_{4}^{\left(2^{s+1}+2^{s}-1\right)}+a_{1}^{\left(2^{s+2}+2^{s+1}-1\right)} a_{2}^{\left(2^{s+2}+2^{s+1}-1\right)} a_{3}^{\left(2^{s+2}+2^{s+1}+2^{s}-1\right)} a_{4}^{\left(2^{s+1}-1\right)} \\
& +a_{1}^{\left(2^{s+2}-1\right)} a_{2}^{\left(2^{s+2}+2^{s+1}-1\right)} a_{3}^{\left(2^{s+3}+2^{s}-1\right)} a_{4}^{\left(2^{s+1}-1\right)}+a_{1}^{\left(2^{s+2}+2^{s+1}-1\right)} a_{2}^{\left(2^{s+2}-1\right)} a_{3}^{\left(2^{s+3}+2^{s}-1\right)} a_{4}^{\left(2^{s+1}-1\right)} \\
& +a_{1}^{\left(2^{s+2}-1\right)} a_{2}^{\left(2^{s+2}+2^{s+1}+2^{s}-1\right)} a_{3}^{\left(2^{s+3}-1\right)} a_{4}^{\left(2^{s+1}-1\right)}+a_{1}^{\left(2^{s+2}+2^{s+1}-1\right)} a_{2}^{\left(2^{s+3}-1\right)} a_{3}^{\left(2^{s+2}+2^{s}-1\right)} a_{4}^{\left(2^{s+1}-1\right)} \\
& +a_{1}^{\left(2^{s+3}-1\right)} a_{2}^{\left(2^{s+2}+2^{s+1}-1\right)} a_{3}^{\left(2^{s+2}+2^{s}-1\right)} a_{4}^{\left(2^{s+1}-1\right)}+a_{1}^{\left(2^{s+2}-1\right)} a_{2}^{\left(2^{s+3}+2^{s+1}-1\right)} a_{3}^{\left(2^{s+2}+2^{s}-1\right)} a_{4}^{\left(2^{s+1}-1\right)} \\
& +a_{1}^{\left(2^{s+3}+2^{s+1}-1\right)} a_{2}^{\left(2^{s+2}-1\right)} a_{3}^{\left(2^{s+2}+2^{s}-1\right)} a_{4}^{\left(2^{s+1}-1\right)}+a_{1}^{\left(2^{s+2}-1\right)} a_{2}^{\left(2^{s+3}+2^{s+1}-1\right)} a_{3}^{\left(2^{s+2}-1\right)} a_{4}^{\left(2^{s+1}+2^{s}-1\right)} \\
& +a_{1}^{\left(2^{s+3}+2^{s+1}-1\right)} a_{2}^{\left(2^{s+2}-1\right)} a_{3}^{\left(2^{s+2}-1\right)} a_{4}^{\left(2^{s+1}+2^{s}-1\right)}+a_{1}^{\left(2^{s+2}+2^{s+1}-1\right)} a_{2}^{\left(2^{s+3}+2^{s+1}-1\right)} a_{3}^{\left(2^{s+1}+2^{s}-1\right)} a_{4}^{\left(2^{s+1}-1\right)} \\
& +a_{1}^{\left(2^{s+3}+2^{s+1}-1\right)} a_{2}^{\left(2^{s+2}+2^{s+1}-1\right)} a_{3}^{\left(2^{s+1}+2^{s}-1\right)} a_{4}^{\left(2^{s+1}-1\right)}+a_{1}^{\left(2^{s+2}+2^{s+1}-1\right)} a_{2}^{\left(2^{s+3}+2^{s+1}+2^{s}-1\right)} a_{3}^{\left(2^{s+1}-1\right)} a_{4}^{\left(2^{s+1}-1\right)} \\
& +a_{1}^{\left(2^{s+3}+2^{s+1}-1\right)} a_{2}^{\left(2^{s+2}+2^{s+1}+2^{s}-1\right)} a_{3}^{\left(2^{s+1}-1\right)} a_{4}^{\left(2^{s+1}-1\right)}+a_{1}^{\left(2^{s+2}-1\right)} a_{2}^{\left(2^{s+3}+2^{s+2}-1\right)} a_{3}^{\left(2^{s+1}+2^{s}-1\right)} a_{4}^{\left(2^{s+1}-1\right)} \\
& +a_{1}^{\left(2^{s+3}+2^{s+2}-1\right)} a_{2}^{\left(2^{s+2}-1\right)} a_{3}^{\left(2^{s+1}+2^{s}-1\right)} a_{4}^{\left(2^{s+1}-1\right)}+a_{1}^{\left(2^{s+2}+2^{s+1}-1\right)} a_{2}^{\left(2^{s+2}+2^{s+1}-1\right)} a_{3}^{\left(2^{s+2}-1\right)} a_{4}^{\left(2^{s+2}+2^{s}-1\right)} \\
& +a_{1}^{\left(2^{s+2}+2^{s+1}-1\right)} a_{2}^{\left(2^{s+2}-1\right)} a_{3}^{\left(2^{s+2}+2^{s+1}-1\right)} a_{4}^{\left(2^{s+2}+2^{s}-1\right)}+a_{1}^{\left(2^{s+2}-1\right)} a_{2}^{\left(2^{s+2}+2^{s+1}-1\right)} a_{3}^{\left(2^{s+2}+2^{s+1}-1\right)} a_{4}^{\left(2^{s+2}+2^{s}-1\right)} \\
& +a_{1}^{\left(2^{s+2}-1\right)} a_{2}^{\left(2^{s+3}+2^{s+2}+2^{s}-1\right)} a_{3}^{\left(2^{s+1}-1\right)} a_{4}^{\left(2^{s+1}-1\right)}+a_{1}^{\left(2^{s+3}+2^{s+2}-1\right)} a_{2}^{\left(2^{s+2}+2^{s}-1\right)} a_{3}^{\left(2^{s+1}-1\right)} a_{4}^{\left(2^{s+1}-1\right)} \\
& +a_{1}^{\left(2^{s+3}-1\right)} a_{2}^{\left(2^{s+3}+2^{s}-1\right)} a_{3}^{\left(2^{s+1}-1\right)} a_{4}^{\left(2^{s+1}-1\right)}+a_{1}^{\left(2^{s+3}-1\right)} a_{2}^{\left(2^{s+3}-1\right)} a_{3}^{\left(2^{s+1}-1\right)} a_{4}^{\left(2^{s+1}+2^{s}-1\right)} \\
& +a_{1}^{\left(2^{s+3}+2^{s+2}+2^{s+1}-1\right)} a_{2}^{\left(2^{s+1}+2^{s}-1\right)} a_{3}^{\left(2^{s+1}-1\right)} a_{4}^{\left(2^{s+1}-1\right)}+a_{1}^{\left(2^{s+3}+2^{s+2}+2^{s+1}+2^{s}-1\right)} a_{2}^{\left(2^{s+1}-1\right)} a_{3}^{\left(2^{s+1}-1\right)} a_{4}^{\left(2^{s+1}-1\right)} \\
& +a_{1}^{\left(2^{s+2}+2^{s+1}+2^{s}-1\right)} a_{2}^{\left(2^{s+2}+2^{s+1}-1\right)} a_{3}^{\left(2^{s+2}-1\right)} a_{4}^{\left(2^{s+2}-1\right)}+a_{1}^{\left(2^{s+2}+2^{s+1}-1\right)} a_{2}^{\left(2^{s+2}-1\right)} a_{3}^{\left(2^{s+2}+2^{s+1}+2^{s}-1\right)} a_{4}^{\left(2^{s+2}-1\right)} \\
& +a_{1}^{\left(2^{s+2}-1\right)} a_{2}^{\left(2^{s+2}+2^{s+1}+2^{s}-1\right)} a_{3}^{\left(2^{s+2}+2^{s+1}-1\right)} a_{4}^{\left(2^{s+2}-1\right)}+a_{1}^{\left(2^{s+2}+2^{s+1}+2^{s}-1\right)} a_{2}^{\left(2^{s+2}-1\right)} a_{3}^{\left(2^{s+2}-1\right)} a_{4}^{\left(2^{s+2}+2^{s+1}-1\right)} \\
& +a_{1}^{\left(2^{s+2}-1\right)} a_{2}^{\left(2^{s+2}-1\right)} a_{3}^{\left(2^{s+2}+2^{s+1}+2^{s}-1\right)} a_{4}^{\left(2^{s+2}+2^{s+1}-1\right)}+a_{1}^{\left(2^{s+2}-1\right)} a_{2}^{\left(2^{s+2}+2^{s+1}+2^{s}-1\right)} a_{3}^{\left(2^{s+2}-1\right)} a_{4}^{\left(2^{s+2}+2^{s+1}-1\right)} \\
& +a_{1}^{\left(2^{s+2}+2^{s+1}-1\right)} a_{2}^{\left(2^{s+2}-1\right)} a_{3}^{\left(2^{s+2}-1\right)} a_{4}^{\left(2^{s+2}+2^{s+1}+2^{s}-1\right)}+a_{1}^{\left(2^{s+2}-1\right)} a_{2}^{\left(2^{s+2}+2^{s+1}-1\right)} a_{3}^{\left(2^{s+2}-1\right)} a_{4}^{\left(2^{s+2}+2^{s+1}+2^{s}-1\right)} \\
& +a_{1}^{\left(2^{s+2}-1\right)} a_{2}^{\left(2^{s+2}-1\right)} a_{3}^{\left(2^{s+2}+2^{s+1}-1\right)} a_{4}^{\left(2^{s+2}+2^{s+1}+2^{s}-1\right)}+a_{1}^{\left(2^{s+2}-1\right)} a_{2}^{\left(2^{s+2}-1\right)} a_{3}^{\left(2^{s+2}-1\right)} a_{4}^{\left(2^{s+3}+2^{s}-1\right)} \\
& +a_{1}^{\left(2^{s+2}-1\right)} a_{2}^{\left(2^{s+2}-1\right)} a_{3}^{\left(2^{s+2}+2^{s}-1\right)} a_{4}^{\left(2^{s+3}-1\right)}+a_{1}^{\left(2^{s+2}-1\right)} a_{2}^{\left(2^{s+2}+2^{s+1}-1\right)} a_{3}^{\left(2^{s+1}+2^{s}-1\right)} a_{4}^{\left(2^{s+3}-1\right)} \\
& +a_{1}^{\left(2^{s+2}-1\right)} a_{2}^{\left(2^{s+2}+2^{s+1}+2^{s}-1\right)} a_{3}^{\left(2^{s+1}-1\right)} a_{4}^{\left(2^{s+3}-1\right)}+a_{1}^{\left(2^{s+2}-1\right)} a_{2}^{\left(2^{s+2}-1\right)} a_{3}^{\left(2^{s+3}+2^{s+1}-1\right)} a_{4}^{\left(2^{s+1}+2^{s}-1\right)} \\
& +a_{1}^{\left(2^{s+2}-1\right)} a_{2}^{\left(2^{s+2}-1\right)} a_{3}^{\left(2^{s+3}+2^{s+1}+2^{s}-1\right)} a_{4}^{\left(2^{s+1}-1\right)}+a_{1}^{\left(2^{s+2}+2^{s+1}-1\right)} a_{2}^{\left(2^{s+2}-1\right)} a_{3}^{\left(2^{s+3}-1\right)} a_{4}^{\left(2^{s+1}+2^{s}-1\right)} \\
& +a_{1}^{\left(2^{s+2}+2^{s+1}-1\right)} a_{2}^{\left(2^{s+2}+2^{s+1}+2^{s}-1\right)} a_{3}^{\left(2^{s+2}-1\right)} a_{4}^{\left(2^{s+1}+2^{s}-1\right)}+a_{1}^{\left(2^{s+2}+2^{s+1}+2^{s}-1\right)} a_{2}^{\left(2^{s+2}+2^{s+1}-1\right)} a_{3}^{\left(2^{s+2}-1\right)} a_{4}^{\left(2^{s+1}+2^{s}-1\right)} .
\end{aligned}
$$

We note also that by our previous work [Ph21c], we deduce that

$$
\left[a_{1}^{\left(2^{s-1}-1\right)} a_{2}^{\left(2^{s-1}-1\right)} a_{3}^{\left(2^{s+2}-1\right)} a_{4}^{\left(2^{s+4}-1\right)}\right]=\left(\left[\varphi^{s-1}(\widehat{\zeta})\right]\right)^{*} \text { for every positive integer } s,
$$

wherein $\widehat{\zeta}=\sum_{1 \leqslant i<j \leqslant 4} x_{i}^{7} x_{j}^{31}+\sum_{1 \leqslant i<j \leqslant 4} x_{i}^{31} x_{j}^{7}+$ other terms. Using the chain-level representation of $\operatorname{Tr}_{4}^{A}$ via lambda algebra, we get

$$
\begin{aligned}
& \operatorname{Tr}_{4}^{A}\left(\left[\zeta_{s}\right]\right)=\left[\psi_{4}\left(\zeta_{s}\right)\right]=\left[\lambda_{2^{s+2}-1} \lambda_{2^{s+2}+2^{s+1}-1}^{2} \lambda_{2^{s+2}+2^{s}-1}+\lambda_{2^{s+3}-1} \lambda_{2^{s+2}+2^{s+1}-1} \lambda_{2^{s+2}-1} \lambda_{2^{s+1}+2^{s}-1}\right. \\
& \quad+\lambda_{2^{s+2}-1}^{2} \lambda_{2^{s+3}-1} \lambda_{2^{s+2}+2^{s}-1}+\lambda_{2^{s+2}-1}^{2} \lambda_{2^{s+2}+2^{s+1}-1} \lambda_{2^{s+2}+2^{s+1}+2^{s}-1}+\lambda_{2^{s+2}-1}^{3} \lambda_{2^{s+3}+2^{s}-1} \\
& \quad+d\left(\lambda_{2^{s+2}+2^{s-1}} \lambda_{2^{s+3}-1}^{2}+\lambda_{2^{s+2}-1} \lambda_{2^{s+2}+2^{s+1}-1} \lambda_{2^{s+3}+2^{s+1}+2^{s}-1}+\lambda_{2^{s+2}-1} \lambda_{2^{s+3}+2^{s+2}+2^{s}-1} \lambda_{2^{s+2}-1}\right. \\
& \left.\left.\quad+\lambda_{2^{s}-1} \lambda_{2^{s+3}+2^{s+2}-1} \lambda_{2^{s+3}-1}\right)\right] \\
& =\left[\lambda_{2^{s+2}-1} \lambda_{2^{s+2}+2^{s+1}-1}^{2} \lambda_{2^{s+2}+2^{s}-1}+\lambda_{2^{s+3}-1} \lambda_{2^{s+2}+2^{s+1}-1} \lambda_{2^{s+2}-1} \lambda_{2^{s+1}+2^{s}-1}\right. \\
& \left.\quad+\lambda_{2^{s+2}-1}^{2} \lambda_{2^{s+3}-1} \lambda_{2^{s+2}+2^{s}-1}+\lambda_{2^{s+2}-1}^{2} \lambda_{2^{s+2}+2^{s+1}-1} \lambda_{2^{s+2}+2^{s+1}+2^{s}-1}+\lambda_{2^{s+2}-1}^{3} \lambda_{2^{s+3}+2^{s}-1}\right]=e_{s}
\end{aligned}
$$

for any $s \geqslant 0$. So, we have immediately

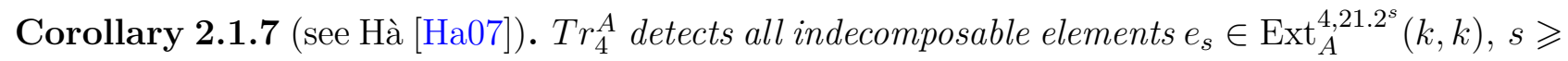
0 .

It must be noted that in [Ha07], Hà employed Boardman's chain-level representation of the algebraic transfer [Bo93], in conjunction with the Sub-Hopf algebra technique, to demonstrate that the element $e_{0}$ lies in the image of $\operatorname{Tr}_{4}^{A}$. (Of course, it is known, the Kameko $S q^{0}$ and the classical $S q^{0}$ commute with each other through the Singer transfer, and so, as $e_{s+1}=S q^{0}\left(e_{s}\right), e_{s} \in \operatorname{Im}\left(\operatorname{Tr}_{4}^{A}\right)$ 
for every s.) But his method does not allow for an explicit determination of a generating set for the domain $k \otimes_{G(4)} P_{A}\left(\left(P_{4}\right)_{21.2^{s}-4}^{*}\right)$ of $\operatorname{Tr}_{4}^{A}$. Therefore, Remark 2.1.6 is of significant importance in this regard. In addition, our technique for determining the structure of the domain of $\operatorname{Tr}_{4}^{A}$ can be applied not only to the stem of $e_{s}$, but also to the stems of $d_{s}, f_{s}$ and $p_{s}$.

Cases $\boldsymbol{t} \geqslant 4$. By Sum [Su14], for each $t \geqslant 4$, the dimension of the $k$-vector spaces $\boldsymbol{Q}_{2^{s+t+1}+2^{s+1}-3}^{4}$ are determined as follows:

$$
\operatorname{dim} Q_{2^{s+t+1}+2^{s+1}-3}^{4}= \begin{cases}150 & \text { if } s=1 \\ 195 & \text { if } s=2 \\ 210 & \text { if } s \geqslant 3\end{cases}
$$

Thanks to these results, by direct calculations, we obtain the following.

Theorem 2.1.8. Let $s, t$ be positive integers such that $t \geqslant 4$. Then,

$$
\operatorname{dim} k \otimes_{G(4)} P_{A}\left(\left(P_{4}\right)_{2^{s+t+1}+2^{s+1}-3}^{*}\right)= \begin{cases}1 & \text { if } s=1,2 \\ 2 & \text { if } s \geqslant 3 .\end{cases}
$$

Moreover,

$$
\begin{aligned}
& k \otimes_{G(4)} P_{A}\left(\left(P_{4}\right)_{2^{s+t+1}+2^{s+1}-3}^{*}\right) \\
& = \begin{cases}\left\langle\left[a_{2}^{\left(2^{s+1}-1\right)} a_{3}^{\left(2^{s+t}-1\right)} a_{4}^{\left(2^{s+t}-1\right)}\right]\right\rangle & \text { if } s=1,2, \\
\left\langle\left[a_{2}^{\left(2^{s+1}-1\right)} a_{3}^{\left(2^{s+t}-1\right)} a_{4}^{\left(2^{s+t}-1\right)}\right],\left[a_{1}^{(0)} a_{2}^{\left(2^{s}-1\right)} a_{3}^{\left(2^{s}-1\right)} a_{4}^{\left(2^{s+t+1}-1\right)}\right]\right\rangle & \text { if } s \geqslant 3 .\end{cases}
\end{aligned}
$$

Due to Theorem 2.1, $\operatorname{Ext}_{A}^{4,2^{s+t+1}+2^{s+1}+1}(k, k)=\left\langle h_{0} h_{s+1} h_{s+t}^{2}, h_{0} h_{s}^{2} h_{s+t+1}\right\rangle$. Noting also that if $t=3$, then $h_{0} h_{s+1} h_{s+t}^{2}=0$. If $t=1$ and $s>2$, then $h_{0} h_{s+1} h_{s+t}^{2}=h_{0} h_{s}^{2} h_{s+t+1}$. For $t=2$ and $s>1$, we have $h_{0} h_{s+1} h_{s+t}^{2}=0$ and $h_{0} h_{s}^{2} h_{s+t+1}=0$. In the case in which $s \in\{1,2\}$, we have $h_{0} h_{s}^{2} h_{s+t+1}=0$. Notice that $a_{2}^{\left(2^{s+1}-1\right)} a_{3}^{\left(2^{s+t}-1\right)} a_{4}^{\left(2^{s+t}-1\right)}$, and $a_{2}^{\left(2^{s}-1\right)} a_{3}^{\left(2^{s}-1\right)} a_{4}^{\left(2^{s+t+1}-1\right)}$ are $\widehat{A}$ annihilated. By this and Remark 2.2, we, consequently, claim that by Theorem 1.2, the cycles

$$
\begin{aligned}
& \lambda_{0} \lambda_{2^{s+1}-1} \lambda_{2^{s+t}-1}^{2}=\psi_{4}\left(a_{2}^{\left(2^{s+1}-1\right)} a_{3}^{\left(2^{s+t}-1\right)} a_{4}^{\left(2^{s+t}-1\right)}\right), \\
& \lambda_{0} \lambda_{2^{s}-1}^{2} \lambda_{2^{s+t+1}-1}=\psi_{4}\left(a_{2}^{\left(2^{s}-1\right)} a_{3}^{\left(2^{s}-1\right)} a_{4}^{\left(2^{s+t+1}-1\right)}\right)
\end{aligned}
$$

in $\Lambda^{4,2^{s+t+1}+2^{s+1}-3}$ are representative of the non-zero elements $h_{0} h_{s+1} h_{s+t}^{2}$ and $h_{0} h_{s}^{2} h_{s+t+1}$, respectively and so, the following is a direct consequence from these data and Theorem 2.1.8.

Corollary 2.1.9. The fourth transfer $\operatorname{Tr}_{4}^{A}$ is an isomorphism in degree $2^{s+t+1}+2^{s+1}-3$ for all $s>0$ and $t>3$.

\subsection{Degree $n=2^{s+t}+2^{s}-2, t \geqslant 1, t \neq 2,3,4$}

The basic strategy for determining $G(4)$-invariants of $\boldsymbol{Q}_{2^{s+t}+2^{s}-2}^{4}$ is as follows: we set up a short exact sequence of $k G(4)$-modules either by using the Kameko homomorphism or the weight vector. These short exact sequence induce a left exact sequence of $G(4)$-invariants. Then, we need to calculate $G(4)$-invariants for the the kernel and quotient $k$-modules in the cases under consideration.

Case $\boldsymbol{t}=1$. For this, $2^{s+t}+2^{s}-2=2^{s+1}+2^{s}-2$, and the Kameko homomorphism

$$
\left[\overline{S q}^{0}\right]_{2^{s+1}+2^{s-2}}:=\overline{S q}^{0}: Q_{2^{s+1}+2^{s}-2}^{4} \longrightarrow Q_{2^{s-1}+2^{s}-3}^{4}
$$

is an epimorphism. So, one has a short exact sequence of $k G(4)$-modules:

$$
0 \longrightarrow \operatorname{Ker}\left(\left[\overline{S q}^{0}\right]_{2^{s+1}+2^{s}-2}\right) \longrightarrow Q_{2^{s+1}+2^{s}-2}^{4} \longrightarrow Q_{2^{s-1}+2^{s}-3}^{4} \longrightarrow 0 .
$$

This induces a left exact sequence of $G(4)$-invariants:

$$
0 \longrightarrow\left(\operatorname{Ker}\left(\left[\overline{S q}^{0}\right]_{2^{s+1}+2^{s}-2}\right)\right)^{G(4)} \longrightarrow\left(\boldsymbol{Q}_{2^{s+1}+2^{s}-2}^{4}\right)^{G(4)} \longrightarrow\left(\boldsymbol{Q}_{2^{s-1}+2^{s}-3}^{4}\right)^{G(4)},
$$


which leads to an estimate

$$
\begin{aligned}
& \operatorname{dim} k \otimes_{G(4)} P_{A}\left(\left(P_{4}\right)_{2^{s+1}+2^{s}-2}^{*}\right)=\operatorname{dim}\left(Q_{2^{s+t}+2^{s}-2}^{4}\right)^{G(4)} \leqslant \operatorname{dim}\left(\operatorname{Ker}\left[\overline{S q}^{0}\right]_{2^{s+1}+2^{s}-2}\right)^{G(4)} \\
& +\operatorname{dim} k \otimes_{G(4)} P_{A}\left(\left(P_{4}\right)_{2^{s-1}+2^{s}-3}^{*}\right) \text {. }
\end{aligned}
$$

Using a monomial basis of $\operatorname{Ker}\left[\overline{S q}^{0}\right]_{2^{s+1}+2^{s-2}}$, we derive

Theorem 2.2.1. The subspace $\left(\operatorname{Ker}\left[\overline{S q}^{0}\right]_{2^{s+1}+2^{s}-2}\right)^{G(4)}$ is trivial for all $s>0$.

Based upon Theorem 2.2.1 and the inequality (8), we could conclude the following. For $s=1$, evidently $Q_{2^{1-1}+2^{1}-3}^{4} \cong k$, and so

$$
k \otimes_{G(4)} P_{A}\left(\left(P_{4}\right)_{2^{1-1}+2^{1}-3}^{*}\right)=\langle[1]\rangle .
$$

Suppose that $[f] \in k \otimes_{G(4)} P_{A}\left(\left(P_{4}\right)_{2^{1+1}+2^{1}-2}^{*}\right)$. Then, $[f]$ is dual to $[\tilde{f}] \in\left(Q_{2^{1+1}+2^{1}-2}^{4}\right)^{G(4)}$. Since the Kameko map $\left[\overline{S q}^{0}\right]_{2^{1+1}+2^{1}-2}$ is an epimorphism, the dual of $\left[\overline{S q}^{0}\right]_{2^{1+1}+2^{1}-2}([\tilde{f}])$ belongs to the coinvariant $k \otimes_{G(4)} P_{A}\left(\left(P_{4}\right)_{2^{1+1}+2^{1}-2}^{*}\right)$. So, due to Theorem 2.2.1 and the equality $(9),[f]$ is dual to $\left(\gamma[\varphi(1)]+\left[\tilde{f}^{\prime}\right]\right)$, wherein $\gamma \in k,\left[\tilde{f}^{\prime}\right] \in \operatorname{Ker}\left[\overline{S q}^{0}\right]_{2^{1+1}+2^{1}-2}$ and the up Kameko map $\varphi$ is determined by

$$
\begin{aligned}
\varphi: k & \longrightarrow\left(P_{4}\right)_{2^{1+1}+2^{1}-2} \\
u & \longmapsto \begin{cases}0 & \text { if } u=0, \\
\prod_{1 \leqslant i \leqslant 4} x_{i} & \text { if } u=1 .\end{cases}
\end{aligned}
$$

By straightforward computations using Lemma 3.4.1(i) (see Section three) and an admissible monomial basis of $Q_{2^{1+1}+2^{1}-2}^{4}$, we get $[\tilde{f}]=0$, which implies that $[f]=([\tilde{f}])^{*}=0$. Hence, the coinvariant $k \otimes_{G(4)} P_{A}\left(\left(P_{4}\right)_{2^{1+1}+2^{1}-2}^{*}\right)$ is trivial. For $s \in\{2,4\}$, by combining Theorems 2.1.1, 2.2.1 with the inequality (8) and the fact that the $G(4)$-invariant $\left(\boldsymbol{Q}_{2^{2-1}+2^{2}-3}\right)^{G(4)}$ is trivial (see Sum [Su21a]), it may be concluded that the coinvariants $k \otimes_{G(4)} P_{A}\left(\left(P_{4}\right)_{2^{s+1}+2^{s}-2}^{*}\right)$ are also trivial. For $s \notin\{1,2,4\}$, the following inequality is immediate from Theorems 2.1.1, 2.2.1 and the inequality (8):

$$
\operatorname{dim} k \otimes_{G(4)} P_{A}\left(\left(P_{4}\right)_{2^{s+1}+2^{s}-2}^{*} \leqslant 1 .\right.
$$

It is routine to verify that the elements

$$
\begin{aligned}
& \zeta_{3}=\left(a_{1}^{(3)} a_{2}^{(7)} a_{3}^{(7)} a_{4}^{(5)}+a_{1}^{(3)} a_{2}^{(7)} a_{3}^{(9)} a_{4}^{(3)}+a_{1}^{(3)} a_{2}^{(11)} a_{3}^{(5)} a_{4}^{(3)}+a_{1}^{(3)} a_{2}^{(13)} a_{3}^{(3)} a_{4}^{(3)}\right) \in\left(P_{4}\right)_{2^{3+1}+2^{3}-2}^{*}, \\
& \zeta_{s}=a_{1}^{(1)} a_{2}^{\left(2^{s-1}-1\right)} a_{3}^{\left(2^{s-1}-1\right)} a_{4}^{\left(2^{s+1}-1\right)} \in\left(P_{4}\right)_{2^{s+1}+2^{s}-2}^{*}, \text { for } s \geqslant 5
\end{aligned}
$$

are $\widehat{A}$-annihilated. Observely $\lambda_{7}^{2} \lambda_{5}=S q^{0}\left(\lambda_{3}^{2} \lambda_{2}\right) \in \Lambda^{3,19}$ is a cycle in $\Lambda$, and is a representative of $c_{1}=S q^{0}\left(c_{0}\right) \in \operatorname{Ext}_{A}^{3,22}(k, k)$. These data together with Remark 2.2 and Theorem 1.2 yield that the cycles $\psi_{4}\left(\zeta_{3}\right)=\lambda_{3} \lambda_{7}^{2} \lambda_{5}$ and $\psi_{4}\left(\zeta_{s}\right)=\lambda_{1} \lambda_{2^{s-1}-1}^{2} \lambda_{2^{s+1}-1}$ in $\Lambda$ are representative of the elements $h_{2} c_{1} \in \operatorname{Ext}_{A}^{4,3.2^{3}+2}(k, k)$ and $h_{1} h_{s-1}^{2} h_{s+1} \in \operatorname{Ext}_{A}^{4,3.2^{s}+2}(k, k)$, respectively. It is also worth noticing that with the $\widehat{A}$-annihilated elements $a^{\left(2^{s}-1\right)} \in\left(P_{1}\right)_{2^{s}-1}^{*}$ and

$$
\widehat{\zeta}=\left(a_{1}^{(7)} a_{2}^{(7)} a_{3}^{(5)}+a_{1}^{(7)} a_{2}^{(9)} a_{3}^{(3)}+a_{1}^{(11)} a_{2}^{(5)} a_{3}^{(3)}+a_{1}^{(13)} a_{2}^{(3)} a_{3}^{(3)}\right) \in\left(P_{3}\right)_{19}^{*},
$$

we have $h_{s}=\operatorname{Tr}_{1}^{A}\left(\left[a_{1}^{\left(2^{s}-1\right)}\right]\right.$ ) and $c_{1}=S q^{0}\left(c_{0}\right)=\operatorname{Tr}_{3}^{A}([\widehat{\zeta}])$ (since the classical $S q^{0}$ commutes with the Kameko $S q^{0}$ via the rank 3 transfer). These arguments and the inequality (10) imply that

$$
\operatorname{dim} k \otimes_{G(4)} P_{A}\left(\left(P_{4}\right)_{2^{s+1}+2^{s}-2}^{*}\right)=1, \text { for } s \notin\{1,2,4\},
$$

and that the Singer transfer is an epimorphism in bidegree $\left(4,3.2^{s}+2\right)$. Moreover, according to Theorem 2.1, we easily get

$$
\operatorname{Ext}_{A}^{4,3.2^{s}+2}(k, k)= \begin{cases}\left\langle h_{1} h_{3}^{3}, h_{2} c_{1}\right\rangle=\left\langle h_{2} c_{1}\right\rangle & \text { if } s=3 \\ 0 & \text { if } s \in\{1,2,4\} \\ \left\langle h_{1} h_{s}^{3}\right\rangle & \text { if } s>4\end{cases}
$$

wherein $h_{1} h_{s}^{3}=h_{1} h_{s-1}^{2} h_{s+1} \neq 0$. So, by (11), we have immediately

Corollary 2.2.2. The Singer algebraic transfer is an isomorphism in bidegree $\left(4,3.2^{s}+2\right)$ for every positive integer $s$. 
Case $\boldsymbol{t} \geqslant \mathbf{5}$. We obtain the following technical theorem.

Theorem 2.2.3. Let $s, t$ be positive integers such that $t \geqslant 5$. Then,

$$
k \otimes_{G(4)} P_{A}\left(\left(P_{4}\right)_{2^{s+t}+2^{s}-2}^{*}\right)= \begin{cases}\left\langle\left[\zeta_{s, t}\right]\right\rangle & \text { if } s=1,2 \\ \left\langle\left[\zeta_{s, t}\right],\left[\widetilde{\zeta}_{s, t}\right]\right\rangle & \text { if } s=3,4 \\ \left\langle\left[\zeta_{s, t}\right],\left[\widetilde{\zeta}_{s, t}\right],\left[\widetilde{\zeta}_{s, t}\right]\right\rangle & \text { if } s \geqslant 5\end{cases}
$$

wherein

$$
\begin{aligned}
\zeta_{s, t} & :=a_{1}^{(1)} a_{2}^{\left(2^{s}-1\right)} a_{3}^{\left(2^{s+t-1}-1\right)} a_{4}^{\left(2^{s+t-1}-1\right)} \\
\widetilde{\zeta}_{s, t} & :=a_{3}^{\left(2^{s}-1\right)} a_{4}^{\left(2^{s+t}-1\right)} \\
\widehat{\zeta}_{s, t} & :=a_{1}^{(1)} a_{2}^{\left(2^{s-1}-1\right)} a_{3}^{\left(2^{s-1}-1\right)} a_{4}^{\left(2^{s+t}-1\right)}
\end{aligned}
$$

The theorem shows that the elements $\zeta_{s, t}, \widetilde{\zeta}_{s, t}$ and $\widehat{\zeta}_{s, t}$ belong to $P_{A}\left(\left(P_{4}\right)_{2^{s+t}+2^{s}-2}^{*}\right)$. So, by Theorem 1.2, $\psi_{4}\left(\zeta_{s, t}\right)$ are cycles in $\Lambda$; moreover using the representation in $\Lambda$ of $\operatorname{Tr}_{4}^{A}$, we get

$$
\begin{aligned}
\operatorname{Tr}_{4}^{A}\left(\left[\zeta_{s, t}\right]\right) & =\left[\psi_{4}\left(\zeta_{s, t}\right)\right]=\left[\lambda_{1} \lambda_{2^{s}-1} \lambda_{2^{s+t-1}-1}^{2}\right] \\
& =h_{1} h_{s} h_{s+t-1}^{2} \in \operatorname{Ext}_{A}^{4,2^{s+t}+2^{s}+2}(k, k), \text { for all } s \geqslant 1, s \neq 2, \\
\operatorname{Tr}_{4}^{A}\left(\left[\widetilde{\zeta}_{s, t}\right]\right) & =\left[\psi_{4}\left(\widetilde{\zeta}_{s, t}\right)\right]=\left[\lambda_{0}^{2} \lambda_{2^{s}-1} \lambda_{2^{s+t}-1}\right] \\
& =h_{0}^{2} h_{s} h_{s+t} \in \operatorname{Ext}_{A}^{42^{s+t}+2^{s}+2}(k, k), \text { for all } s \geqslant 2, \\
\operatorname{Tr}_{4}^{A}\left(\left[\widehat{\zeta}_{s, t}\right]\right) & =\left[\psi_{4}\left(\widetilde{\zeta}_{s, t}\right)\right]=\left[\lambda_{1} \lambda_{2^{s-1}-1}^{2} \lambda_{2^{s+t}-1}\right] \\
& =h_{1} h_{s-1}^{2} h_{s+t} \in \operatorname{Ext}_{A}^{4,2^{s+t}+2^{s}+2}(k, k), \text { for all } s \geqslant 5 .
\end{aligned}
$$

On the other side, by Theorem 2.1,

$$
\operatorname{Ext}_{A}^{4,2^{s+t}+2^{s}+2}(k, k)= \begin{cases}\left\langle h_{1}^{2} h_{7}^{2}, D_{3}(2)\right\rangle & \text { if } s=1 \text { and } t=7, \\ \left\langle h_{1}^{2} h_{t}^{2}\right\rangle & \text { if } s=1 \text { and } t \geqslant 5, t \neq 7, \\ \left\langle h_{0}^{2} h_{2} h_{t+2}\right\rangle=\left\langle h_{1}^{3} h_{t+2}\right\rangle & \text { if } s=2 \text { and } t \geqslant 5, \\ \left\langle h_{1} h_{s} h_{s+t-1}^{2}, h_{0}^{2} h_{s} h_{s+t}, h_{1} h_{s-1}^{2} h_{s+t}\right\rangle & \text { if } s \geqslant 3 \text { and } t \geqslant 5\end{cases}
$$

wherein $h_{1} h_{s-1}^{2} h_{s+t}=0$ if $s=3,4$, and $t \geqslant 5$. Since $\operatorname{Tr}_{4}^{A}\left(\left[\zeta_{1,7}\right]\right)=h_{1}^{2} h_{7}^{2}$, by Theorem 2.2 .3 , the indecomposable element $D_{3}(2)$ is not in the image of $\operatorname{Tr}_{4}^{A}$. As a valuable consequence, we derive

Corollary 2.2.4. The transfer homomorphism

$$
\operatorname{Tr}_{4}^{A}: k \otimes_{G(4)} P_{A}\left(\left(P_{4}\right)_{2^{s+t}+2^{s}-2}^{*}\right) \longrightarrow \operatorname{Ext}_{A}^{4,2^{s+t}+2^{s}+2}(k, k)
$$

is not an epimorphism if $s=1, t=7$, and is an isomorphism if $s \geqslant 1, t \geqslant 5, t \neq 7$.

Remark 2.2.5. Knowing that the iterated Kameko map $\left(\overline{S q}^{0}\right)^{s-2}: \boldsymbol{Q}_{65.2^{s-4}}^{4} \longrightarrow \boldsymbol{Q}_{256}^{4}$ is an isomorphism of $k G(4)$-modules for any $s \geqslant 2$. Thanks to this together with Theorem 2.2.3 and Sum's calculations [Su18] show that

$$
k \otimes_{G(4)} P_{A}\left(\left(P_{4}\right)_{65.2^{s}-4}^{*}\right)= \begin{cases}0 & \text { if } s=0 \text { (see Sum [Su18]) } \\ k\left[a_{3}^{(63)} a_{4}^{(63)}\right] & \text { if } s=1 \text { (see Sum [Su18]) } \\ k\left[\zeta_{1,7}\right] & \text { if } s=2 \text { (see Theorem 2.2.3) } \\ k\left[a_{1}^{\left(2^{s-1}-1\right)} a_{2}^{\left(2^{s-1}-1\right)} a_{3}^{\left(2^{s+5}-1\right)} a_{4}^{\left(2^{s+5}-1\right)}\right] & \text { if } s \geqslant 3,\end{cases}
$$

and so, $k \otimes_{G(4)} P_{A}\left(\left(P_{4}\right)_{65.2^{s}-4}^{*}\right)=k\left[a_{1}^{\left(2^{s-1}-1\right)} a_{2}^{\left(2^{s-1}-1\right)} a_{3}^{\left(2^{s+5}-1\right)} a_{4}^{\left(2^{s+5}-1\right)}\right]$ for arbitrary $s \geqslant 1$. Using the $E_{1}$-level of Singer's transfer, we must have that

$$
\operatorname{Tr}_{4}^{A}\left(\left[a_{1}^{\left(2^{s-1}-1\right)} a_{2}^{\left(2^{s-1}-1\right)} a_{3}^{\left(2^{s+5}-1\right)} a_{4}^{\left(2^{s+5}-1\right)}\right]\right)=\left[\psi_{4}\left(a_{1}^{\left(2^{s-1}-1\right)} a_{2}^{\left(2^{s-1}-1\right)} a_{3}^{\left(2^{s+5}-1\right)} a_{4}^{\left(2^{s+5}-1\right)}\right)\right]=h_{s-1}^{2} h_{s+5}^{2},
$$

for every $s>0$. On the other hand, according to Theorem 2.1,

$$
\operatorname{Ext}_{A}^{4,65.2^{s}}(k, k)= \begin{cases}\left\langle D_{3}(0)\right\rangle & \text { if } s=0 \\ \left\langle D_{3}(s), h_{s-1}^{2} h_{s+5}^{2}\right\rangle & \text { if } s \geqslant 1\end{cases}
$$

We thereby can confirm the following consequence, which was demonstrated by Hưng [Hu05, Theorem 7.3] through computer calculations. 
Corollary 2.2.6. Every element in the $S q^{0}$-family $\left\{D_{3}(s): s \geqslant 0\right\}$ does not belong to the image of the fourth transfer homomorphism.

By virtue of Corollaries 2.1.3, 2.1.5, 2.1.9, 2.2.2, and 2.2.4, we have derived the following.

Corollary 2.2.7. Let $s, t$ be two positive integers. Then, Singer's Conjecture 1.1 holds for $q=4$ in degrees $2^{s+t+1}+2^{s+1}-3, t \neq 3$, and degrees $2^{s+t}+2^{s}-2, t \neq 2,3,4$.

\section{Proofs of main results}

Note 3.1. The entire section is dedicated to proving Theorems 2.1.1, 2.1.4, 2.1.8, 2.2.1, and 2.2.3. Our approach in demonstrating the main results involves utilizing Sum's list [Su14] of generators for $\boldsymbol{Q}_{n}^{4}$ in generic degrees $n$. We then compute the $G(4)$-coinvariants of $\boldsymbol{Q}_{n}^{4}$ by first finding its $\Sigma_{4}$-invariants, dualizing each element of $\left(\boldsymbol{Q}_{n}^{4}\right)^{G(4)}$, and using the $E_{1}$-level of Singer's transfer to determine its image. The challenge in executing this construction lies in explicitly representing inadmissible elements as linear combinations of admissible terms (modulo $A$-decomposables in $P_{4}$ ). These calculations are often difficult to perform, and as such, we are nearly required to re-solve the hit problem for $P_{4}$. To achieve this, we employ a combination of manual computation and computer programs. In actuality, this is necessary because Sum's paper [Su15] only thoroughly addressed two special cases of the form (3) and omitted the proof of the remaining cases, referring to [Su14] instead. Furthermore, as [Su14] is still a preprint, we also utilized a computer algorithm [Ph21b, Appendix] to confirm the dimension results for $\boldsymbol{Q}_{n}^{4}$. Of course, it is possible that there may be advanced computer programs, using current techniques, that could re-create Sum's manual calculations [Su14]. Nevertheless, this would likely depend on the complexity and level of detail of Sum's calculations, as well as the specific computer programs and techniques available. In some cases, manual calculations may still be necessary, particularly if the underlying mathematical concepts are not well understood or if the calculations are too complex for current computer programs to handle. Ultimately, the feasibility of using a computer program to re-create Sum's manual calculations would need to be evaluated on a case-by-case basis.

Now, before presenting the proofs of our main results, let us briefly review some background information in [Ka90, Ph20, Su15].

- A sequence of non-negative integers $\omega=\left(\omega_{1}, \omega_{2}, \ldots, \omega_{i}, \ldots\right)$ is called a weight vector, if $\omega_{i}=0$ for $i \gg 0$. One defines $\operatorname{deg}(\omega)=\sum_{i \geqslant 1} 2^{i-1} \omega_{i}$. For a natural number $n$, let us denote by $\alpha_{j}(n)$ the $j$-th coefficients in dyadic expansion of $n$, from which we have $\alpha(n)=\sum_{j \geqslant 0} \alpha_{j}(n)$ and $n=$ $\sum_{j \geqslant 0} \alpha_{j}(n) 2^{j}$, wherein $\alpha_{j}(n) \in\{0,1\}, j=0,1, \ldots$ Let $x=\prod_{1 \leqslant i \leqslant 4} x_{i}^{a_{i}}$ be a monomial in $P_{4}$, define two sequences associated with $x$ by $\omega(x):=\left(\omega_{1}(x), \omega_{2}(x), \ldots, \omega_{j}(x), \ldots\right)$ and $\left(a_{1}, a_{2}, \ldots, a_{4}\right)$, wherein $\omega_{j}(x)=\sum_{1 \leqslant i \leqslant 4} \alpha_{j-1}\left(a_{i}\right)$, for $j \geqslant 1$. These sequences are respectively called the weight vector and the exponent vector of $x$. By convention, the sets of all the weight vectors and the exponent vectors are given the left lexicographical order.

- Given the monomials $x=\prod_{1 \leqslant i \leqslant 4} x_{i}^{a_{i}}$ and $y=\prod_{1 \leqslant i \leqslant 4} x_{i}^{b_{i}}$ in $\left(P_{4}\right)_{n}$, write $a$ and $b$ for the exponent vectors of $x$ and $y$, respectively. We say that $x<y$ if and only if one of the following holds: (i) $\omega(x)<\omega(y)$; (ii) $\omega(x)=\omega(y)$ and $a<b$.

- We denote two $k$-subspaces associated with a weight vector $\omega$ of degree $n$ by $\left(P_{4}\right)_{n}^{\omega}=\langle\{x \in$ $\left.\left.\left(P_{4}\right)_{n} \mid \omega(x) \leqslant \omega\right\}\right\rangle$ and $\left(P_{4}\right)_{n}^{<\omega}=\left\langle\left\{x \in\left(P_{4}\right)_{n} \mid \omega(x)<\omega\right\}\right\rangle$. Clearly these spaces are not $A$ submodules of $P_{4}$. Given the homogeneous polynomials $f$ and $g$ in $\left(P_{4}\right)_{n}$, the following equivalence relations " $\equiv "$ and " $\equiv_{\omega}$ " on $\left(P_{4}\right)_{n}$ are well-defined:

(i) $f \equiv g$ if and only if $(f-g) \in \widehat{A}\left(P_{4}\right)_{n}$;

(ii) $f \equiv_{\omega} g$ if and only if $f, g \in\left(P_{4}\right)_{n}^{\omega}$ and $(f-g) \in\left(\left(\widehat{A}\left(P_{4}\right)_{n} \cap\left(P_{4}\right)_{n}^{\omega}\right)+\left(P_{4}\right)_{n}^{<\omega}\right)$.

In particular, if $f \equiv 0$ (resp. $f \equiv \omega 0$ ), then $f$ is called hit (resp. $\omega$-hit).

Then, by the equivalence relation " $\equiv \omega^{\prime}$ ", one has a quotient

$$
\left(\boldsymbol{Q}_{n}^{4}\right)^{\omega}:=\left(P_{4}\right)_{n}^{\omega} /\left(\left(\left(P_{4}\right)_{n}^{\omega} \cap \widehat{A}\left(P_{4}\right)_{n}\right)+\left(P_{4}\right)_{n}^{<\omega}\right) .
$$

For the reader's convenience, we write $[f]$ for the class in $Q_{n}^{4}$ represented by $f$. If $f \in\left(P_{4}\right)_{n}^{\omega}$, then denote by $[f]_{\omega}$ the class in $\left(\boldsymbol{Q}_{n}^{4}\right)^{\omega}$ represented by $f$. According to Sum [Su21b], this $\left(\boldsymbol{Q}_{n}^{4}\right)^{\omega}$ has the 
structure of an $G(4)$-module for any $n>0$. Furthermore, it is straightforward to show that

$$
\operatorname{dim} \boldsymbol{Q}_{n}^{4}=\sum_{\operatorname{deg}(\omega)=n} \operatorname{dim}\left(\boldsymbol{Q}_{n}^{4}\right)^{\omega}, \quad \text { and } \operatorname{dim}\left(\boldsymbol{Q}_{n}^{4}\right)^{G(4)} \leqslant \sum_{\operatorname{deg}(\omega)=n} \operatorname{dim}\left(\left(\boldsymbol{Q}_{n}^{4}\right)^{\omega}\right)^{G(4)} .
$$

- We denote by $\underline{P_{4}}$ and $\overline{P_{4}}$ the $A$-submodules of $P_{4}$ spanned by all the monomials $\prod_{1 \leqslant i \leqslant 4} x_{i}^{a_{i}}$ such that $\prod_{1 \leqslant i \leqslant 4} a_{i}=0$, and $\prod_{1 \leqslant i \leqslant 4} a_{i}>0$, respectively. Then, $\underline{P_{4}}=\bigoplus_{n \geqslant 0}\left(\underline{P_{4}}\right)_{n}$ and $\overline{P_{4}}=\bigoplus_{n \geqslant 0}\left(\overline{P_{4}}\right)_{n}$. In each $n \geqslant 0$, set $\underline{Q_{n}^{4}}=\left\langle\left\{[f] \in Q_{n}^{4}: f \in\left(\underline{P_{4}}\right)_{n}\right\}\right\rangle$ and $\overline{Q_{n}^{4}}=\left\langle\left\{[f] \in Q_{n}^{4}: f \in\left(\overline{P_{4}}\right)_{n}\right\}\right\rangle$. Then, one has an isomorphism: $\boldsymbol{Q}_{n}^{4} \cong \underline{Q}_{n}^{4} \oplus \overline{Q_{n}^{4}}$. If $\omega$ is a weight vector of degree $n$, then we set $\left({\underline{Q_{n}^{4}}}^{4}\right)^{\omega}=\left\langle\left\{[f]_{\omega} \in\left(\boldsymbol{Q}_{n}^{4}\right)^{\omega}: f \in\left(\underline{P_{4}}\right)_{n}^{\omega}\right\}\right\rangle$, and $\left(\overline{\boldsymbol{Q}_{n}^{4}}\right)^{\omega}=\left\langle\left\{[f]_{\omega} \in\left(\boldsymbol{Q}_{n}^{4}\right)^{\omega}: f \in\left(\overline{P_{4}}\right)_{n}^{\omega}\right\}\right\rangle$.

- A monomial $x \in\left(P_{4}\right)_{n}$ is said to be inadmissible if there exist monomials $y_{1}, y_{2}, \ldots, y_{k}$ in $\left(P_{4}\right)_{n}$ such that $y_{j}<x$ for $1 \leqslant j \leqslant k$ and $x \equiv \sum_{1 \leqslant j \leqslant k} y_{j}$. Then, $x$ is said to be admissible if it is not inadmissible.

Thus it is easily seen that $\boldsymbol{Q}_{n}^{4}$ is a $k$-vector space with a basis consisting of all classes represented by admissible monomials in $\left(P_{4}\right)_{n}$.

- A monomial $z=x_{1}^{v_{1}} \ldots x_{4}^{v_{4}}$ in $\left(P_{4}\right)_{n}$ is called a spike if every exponent $v_{j}$ is of the form $2^{\xi_{j}}-1$. In particular, if the exponents $\xi_{j}$ can be arranged to satisfy $\xi_{1}>\xi_{2}>\ldots>\xi_{m-1} \geqslant \xi_{m} \geqslant 1$ wherein only the last two smallest exponents can be equal, and $\xi_{r}=0$ for $m<r \leqslant 4$, then $z$ is called a minimal spike.

The following technical result is due to Singer [Si89].

Theorem 3.2. Given any $n \geqslant 1$ with $\mu(n) \leqslant 4$, let $z$ be a minimal spike in $\left(P_{4}\right)_{n}$. Then all monomials $x$ in $\left(P_{4}\right)_{n}$ are hit if $\omega(x)<\omega(z)$.

- For each $1 \leqslant j \leqslant 4$, we define the $k$-homomorphism $\sigma_{j}: k^{4} \longrightarrow k^{4}$ by

$\left\{\begin{array}{ll}\sigma_{j}\left(x_{j}\right) & =x_{j+1}, \\ \sigma_{j}\left(x_{j+1}\right) & =x_{j}, \\ \sigma_{j}\left(x_{t}\right) & =x_{t},\end{array}\right.$ for $t \notin\{j, j+1\}, 1 \leqslant j \leqslant 3$, and $\sigma_{4}\left(x_{1}\right)=x_{1}+x_{2}, \sigma_{4}\left(x_{i}\right)=x_{i}$ for $2 \leqslant i \leqslant 4$.

Hereafter, we write $\Sigma_{4}$ as the symmetric group of rank 4 . Then, $\Sigma_{4}$ is generated by the ones associated with $\sigma_{j}, 1 \leqslant j \leqslant 3$. For each permutation in $\Sigma_{4}$, consider corresponding permutation matrix; these form a group of matrices isomorphic to $\Sigma_{4}$. So, $G(4) \cong G L\left(k^{4}\right)$ is generated by the matrices associated with $\sigma_{j}, 1 \leqslant j \leqslant 4$. Let $x=\prod_{1 \leqslant i \leqslant 4} x_{i}^{a_{i}}$ be an monomial in $\left(P_{4}\right)_{n}$. Then, the weight vector $\omega$ of $x$ is invariant under the permutation of the generators $x_{i}, i=1,2, \ldots, 4$. Hence $\left(\boldsymbol{Q}_{n}^{4}\right)^{\omega}$ also has a $\Sigma_{4}$-module structure. As we have seen, $\sigma_{j}$ induces a homomorphism of $A$-algebras which is also denoted by $\sigma_{j}: P_{4} \longrightarrow P_{4}$. So, an element $[f]_{\omega} \in\left(Q_{n}^{4}\right)^{\omega}$ is an $G(4)$-invariant if and only if $\sigma_{j}(f) \equiv_{\omega} f$ for $1 \leqslant j \leqslant 4$. It is an $\Sigma_{4}$-invariant if and only if $\sigma_{j}(f) \equiv_{\omega} f$ for $1 \leqslant j \leqslant 3$.

- In what follows, for any monomials $v_{1}, v_{2}, \ldots, v_{s} \in\left(P_{4}\right)_{n}$ and for a subgroup $G$ of $G(4)$, we denote by $G\left(v_{1} ; v_{2} ; \ldots, v_{s}\right)$ the $G$-submodule of $\left(\boldsymbol{Q}_{n}^{4}\right)^{\omega}$ generated by the set $\left\{\left[v_{i}\right]_{\omega}: 1 \leqslant i \leqslant s\right\}$. It is to be noted that if $\omega$ is a weight vector of a minimal spike, then $\left[v_{i}\right]_{\omega}=\left[v_{i}\right]$ for all $i$. Denote by $<-,->$ the dual pairing between $H_{*}\left(k^{4}, k\right)=\left(P_{4}\right)^{*}$ and $H^{*}\left(k^{4}, k\right)=P_{4}$. In particular, $<a_{1}^{\left(i_{1}\right)} a_{2}^{\left(i_{2}\right)} \ldots a_{4}^{\left(i_{4}\right)}, x_{1}^{i_{1}^{\prime}} x_{2}^{i_{2}^{\prime}} \ldots x_{4}^{i_{4}^{\prime}}>$ is 1 if $i_{j}=i_{j}^{\prime}$ for every $j$, and is 0 otherwise.

\subsection{Proof of Theorem 2.1.1}

For convenience, put $n_{s}:=2^{s+2}+2^{s+1}-3$, and according to Sum [Su14], we have

$$
\operatorname{dim} Q_{n_{s}}^{4}= \begin{cases}46 & \text { if } s=1 \\ 94 & \text { if } s=2 \\ 105 & \text { if } s \geqslant 3\end{cases}
$$

Recall that $\boldsymbol{Q}_{n_{s}}^{4} \cong Q_{n_{s}}^{4} \oplus \overline{Q_{n_{s}}^{4}}$. By Sum [Su15, Su21a], the basis for $\boldsymbol{Q}_{n_{s}}^{4}$ is a set consisting of all the classes represented by the following admissible monomials: 


$$
\begin{array}{ll}
a_{s, 1}=x_{2}^{2^{s}-1} x_{3}^{2^{s}-1} x_{4}^{2^{s+2}-1}, & a_{s, 2}=x_{2}^{2^{s}-1} x_{3}^{2^{s+2}-1} x_{4}^{2^{s}-1}, \\
a_{s, 4}=x_{1}^{2^{s}-1} x_{3}^{2^{s}-1} x_{4}^{2^{s+2}-1}, & a_{s, 5}=x_{1}^{2^{s}-1} x_{3}^{2^{s+2}-1} x_{4}^{2^{s}-1}, \\
a_{s, 7}=x_{1}^{2^{s}-1} x_{2}^{2^{s}-1} x_{3}^{2^{s+2}-1}, & a_{s, 8}=x_{1}^{2^{s}-1} x_{2}^{2^{s+2}-1} x_{4}^{2^{s}-1}, \\
a_{s, 10}=x_{1}^{2^{s+2}-1} x_{3}^{2^{s}-1} x_{4}^{2^{s}-1}, & a_{s, 11}=x_{1}^{2^{s+2}-1} x_{2}^{2^{s}-1} x_{4}^{2^{s}-1}, \\
a_{s, 13}=x_{2}^{2^{s}-1} x_{3}^{2^{s+1}-1} x_{4}^{3.2^{s}-1}, & a_{s, 14}=x_{2}^{2^{s+1}-1} x_{3}^{2^{s}-1} x_{4}^{3.2^{s}-1}, \\
a_{s, 16}=x_{1}^{2^{s}-1} x_{3}^{2^{s+1}-1} x_{4}^{3.2^{s}-1}, & a_{s, 17}=x_{1}^{2^{s}-1} x_{2}^{2^{s+1}-1} x_{4}^{3.2^{s}-1}, \\
a_{s, 19}=x_{1}^{2^{s+1}-1} x_{3}^{2^{s}-1} x_{4}^{3.2^{s}-1}, & a_{s, 20}=x_{1}^{2^{s+1}-1} x_{3}^{3.2^{s}-1} x_{4}^{2^{s}-1}, \\
a_{s, 22}=x_{1}^{2^{s+1}-1} x_{2}^{2^{s}-1} x_{3}^{3.2^{s}-1}, & a_{s, 23}=x_{1}^{2^{s+1}-1} x_{2}^{3.2^{s}-1} x_{4}^{2^{s}-1}, \\
a_{s, 25}=x_{2}^{2^{s+1}-1} x_{3}^{2^{s+1}-1} x_{4}^{2^{s+1}-1}, & a_{s, 26}=x_{1}^{2^{s+1}-1} x_{3}^{2^{s+1}-1} x_{4}^{2^{s+1}-1}, \\
a_{s, 28}=x_{1}^{2^{s+1}-1} x_{2}^{2^{s+1}-1} x_{3}^{2^{s+1}-1} . &
\end{array}
$$$$
a_{s, 3}=x_{2}^{2^{s+2}-1} x_{3}^{2^{s}-1} x_{4}^{2^{s}-1},
$$$$
a_{s, 6}=x_{1}^{2^{s}-1} x_{2}^{2^{s}-1} x_{4}^{2^{s+2}-1},
$$$$
a_{s, 9}=x_{1}^{2^{s}-1} x_{2}^{2^{s+2}-1} x_{3}^{2^{s}-1} \text {, }
$$$$
a_{s, 12}=x_{1}^{2^{s+2}-1} x_{2}^{2^{s}-1} x_{3}^{2^{s}-1},
$$$$
a_{s, 15}=x_{2}^{2^{s+1}-1} x_{3}^{3.2^{s}-1} x_{4}^{2^{s}-1} \text {, }
$$$$
a_{s, 18}=x_{1}^{2^{s}-1} x_{2}^{2^{s+1}-1} x_{3}^{3.2^{s}-1} \text {, }
$$$$
a_{s, 21}=x_{1}^{2^{s+1}-1} x_{2}^{2^{s}-1} x_{4}^{3.2^{s}-1} \text {, }
$$$$
a_{s, 24}=x_{1}^{2^{s+1}-1} x_{2}^{3.2^{s}-1} x_{3}^{2^{s}-1} \text {, }
$$$$
a_{s, 27}=x_{1}^{2^{s+1}-1} x_{2}^{2^{s+1}-1} x_{4}^{2^{s+1}-1} \text {, }
$$

Thanks to these, a direct computation shows that

$$
\begin{aligned}
& \Sigma_{4}\left(a_{s, 1}\right)=\left\langle\left\{\left[a_{s, j}\right]: 1 \leqslant j \leqslant 12\right\}\right\rangle, \\
& \Sigma_{4}\left(a_{s, 13}\right)=\left\langle\left\{\left[a_{s, j}\right]: 13 \leqslant j \leqslant 24\right\}\right\rangle, \\
& \Sigma_{4}\left(a_{s, 25}\right)=\left\langle\left\{\left[a_{s, j}\right]: 25 \leqslant j \leqslant 28\right\}\right\rangle .
\end{aligned}
$$

These imply that there is a direct summand decomposition of the $\Sigma_{4}$-modules:

$$
\underline{\boldsymbol{Q}_{n_{s}}^{4}}=\Sigma_{4}\left(a_{s, 1}\right) \bigoplus \Sigma_{4}\left(a_{s, 13}\right) \bigoplus \Sigma_{4}\left(a_{s, 25}\right) .
$$

In the following, it will play a key role in the proof of the theorem.

Lemma 3.1.1. With the notations above,
i) $\Sigma_{4}\left(a_{s, 1}\right)^{\Sigma_{4}}=\left\langle\left[q_{s, 1}\right]\right\rangle$, with $q_{s, 1}=\sum_{1 \leqslant j \leqslant 12} a_{s, j}$;
ii) $\Sigma_{4}\left(a_{s, 13}\right)^{\Sigma_{4}}=\left\langle\left[q_{s, 2}\right]\right\rangle$, with $q_{s, 1}=\sum_{13 \leqslant j \leqslant 24} a_{s, j}$;
iii) $\Sigma_{4}\left(a_{s, 25}\right)^{\Sigma_{4}}=\left\langle\left[q_{s, 3}\right]\right\rangle$, with $q_{s, 1}=\sum_{25 \leqslant j \leqslant 28} a_{s, j}$.

Proof. We frist prove Part $i)$. Since the set $\left\{\left[a_{s, j}\right]: 1 \leqslant j \leqslant 12\right\}$ is a basis of $\Sigma_{4}\left(a_{s, 1}\right)$, if $[f] \in$ $\Sigma_{4}\left(a_{s, 1}\right)^{\Sigma_{4}}$, then

$$
\begin{aligned}
f \equiv & \left(\gamma_{1} a_{s, 1}+\gamma_{2} a_{s, 2}+\gamma_{3} a_{s, 3}+\gamma_{4} a_{s, 4}+\gamma_{5} a_{s, 5}+\gamma_{6} a_{s, 6}\right. \\
& \left.\gamma_{7} a_{s, 7}+\gamma_{8} a_{s, 8}+\gamma_{9} a_{s, 9}+\gamma_{10} a_{s, 10}+\gamma_{11} a_{s, 11}+\gamma_{12} a_{s, 12}\right),
\end{aligned}
$$

in which $\gamma_{j} \in k$ for every $j$. Acting the homomorphisms $\sigma_{i}: P_{4} \longrightarrow P_{4}, i=1,2,3$, on both sides of (13), we get

$$
\begin{aligned}
\sigma_{1}(f) \equiv & \left(\gamma_{4} a_{s, 1}+\gamma_{5} a_{s, 2}+\gamma_{10} a_{s, 3}+\gamma_{1} a_{s, 4}+\gamma_{2} a_{s, 5}+\gamma_{6} a_{s, 6}+\gamma_{7} a_{s, 7}\right. \\
& \left.+\gamma_{11} a_{s, 8}+\gamma_{12} a_{s, 9}+\gamma_{3} a_{s, 10}+\gamma_{8} a_{s, 11}+\gamma_{9} a_{s, 12}\right) \\
\sigma_{2}(f) \equiv & \left(\gamma_{1} a_{s, 1}+\gamma_{3} a_{s, 2}+\gamma_{2} a_{s, 3}+\gamma_{6} a_{s, 4}+\gamma_{8} a_{s, 5}+\gamma_{4} a_{s, 6}+\gamma_{9} a_{s, 7}\right. \\
& \left.+\gamma_{5} a_{s, 8}+\gamma_{7} a_{s, 9}+\gamma_{11} a_{s, 10}+\gamma_{10} a_{s, 11}+\gamma_{12} a_{s, 12}\right) . \\
\sigma_{3}(f) \equiv & \left(\gamma_{2} a_{s, 1}+\gamma_{1} a_{s, 2}+\gamma_{3} a_{s, 3}+\gamma_{5} a_{s, 4}+\gamma_{4} a_{s, 5}+\gamma_{7} a_{s, 6}+\gamma_{6} a_{s, 7}\right. \\
& \left.+\gamma_{9} a_{s, 8}+\gamma_{8} a_{s, 9}+\gamma_{10} a_{s, 10}+\gamma_{12} a_{s, 11}+\gamma_{11} a_{s, 12}\right) .
\end{aligned}
$$

Since $[f] \in \Sigma_{4}\left(a_{s, 1}\right)^{\Sigma_{4}}, \sigma_{i}(f) \equiv f$ for $1 \leqslant i \leqslant 3$. Combining this and the equalities (13) and (14), we find that

$$
\begin{aligned}
& \left(\gamma_{1}+\gamma_{4}\right) a_{s, 1}+\left(\gamma_{2}+\gamma_{5}\right) a_{s, 2}+\left(\gamma_{3}+\gamma_{10}\right) a_{s, 3}+\left(\gamma_{8}+\gamma_{11}\right) a_{s, 8}+\left(\gamma_{9}+\gamma_{12}\right) a_{s, 9} \equiv 0, \\
& \left(\gamma_{2}+\gamma_{3}\right) a_{s, 2}+\left(\gamma_{4}+\gamma_{6}\right) a_{s, 4}+\left(\gamma_{5}+\gamma_{8}\right) a_{s, 5}+\left(\gamma_{7}+\gamma_{9}\right) a_{s, 7}+\left(\gamma_{10}+\gamma_{11}\right) a_{s, 10} \equiv 0, \\
& \left(\gamma_{1}+\gamma_{2}\right) a_{s, 1}+\left(\gamma_{4}+\gamma_{5}\right) a_{s, 4}+\left(\gamma_{6}+\gamma_{7}\right) a_{s, 6}+\left(\gamma_{8}+\gamma_{9}\right) a_{s, 8}+\left(\gamma_{11}+\gamma_{12}\right) a_{s, 11} \equiv 0 .
\end{aligned}
$$

This implies that $\gamma_{1}=\gamma_{j}$ for all $j, 2 \leqslant j \leqslant 12$. By a similar computation, we also get Part iii). 
We now prove Part $i i)$. We see that a basis for $\Sigma_{4}\left(a_{s, 13}\right)$ is the set $\left\{\left[a_{s, j}\right]: 13 \leqslant j \leqslant 24\right\}$. Assume that $[g] \in \Sigma_{4}\left(a_{s, 13}\right)^{\Sigma_{4}}$, then we have

$$
g \equiv \sum_{13 \leqslant j \leqslant 24} \beta_{j} a_{s, j}
$$

with $\beta_{j} \in k, j=13, \ldots, 24$. Acting the homomorphisms $\sigma_{i}: P_{4} \longrightarrow P_{4}, 1 \leqslant i \leqslant 3$, on both sides of (15), we get

$$
\begin{aligned}
\sigma_{1}(g) \equiv & \left(\beta_{16} a_{s, 13}+\beta_{19} a_{s, 14}+\beta_{20} a_{s, 15}+\beta_{13} a_{s, 16}+\beta_{21} a_{s, 17}\right. \\
& +\beta_{22} a_{s, 18}+\beta_{14} a_{s, 19}+\beta_{15} a_{s, 20}+\beta_{17} a_{s, 21}+\beta_{18} a_{s, 22} \\
& \left.+\beta_{23} x_{1}^{32^{s}-1} x_{2}^{2^{s+1}-1} x_{4}^{2^{s}-1}+\beta_{24} x_{1}^{3.2^{s}-1} x_{2}^{2^{s+1}-1} x_{3}^{2^{s}-1}\right) ; \\
\sigma_{2}(g) \equiv & \left(\beta_{14} a_{s, 13}+\beta_{13} a_{s, 14}+\beta_{17} a_{s, 16}+\beta_{16} a_{s, 17}+\beta_{21} a_{s, 19}\right. \\
& +\beta_{23} a_{s, 20}+\beta_{19} a_{s, 21}+\beta_{24} a_{s, 22}+\beta_{20} a_{s, 23}+\beta_{22} a_{s, 24} \\
& \left.+\beta_{15} x_{2}^{3.2^{s}-1} x_{3}^{2^{s+1}-1} x_{4}^{2^{s}-1}+\beta_{18} x_{1}^{2^{s}-1} x_{2}^{3.2^{s}-1} x_{3}^{2^{s+1}-1}\right) ; \\
\sigma_{3}(g) \equiv & \left(\beta_{15} a_{s, 14}+\beta_{14} a_{s, 15}+\beta_{18} a_{s, 17}+\beta_{17} a_{s, 18}+\beta_{20} a_{s, 19}\right. \\
& +\beta_{19} a_{s, 20}+\beta_{22} a_{s, 21}+\beta_{21} a_{s, 22}+\beta_{24} a_{s, 23}+\beta_{23} a_{s, 24} \\
& \left.+\beta_{13} x_{2}^{2^{s}-1} x_{3}^{3.2^{s}-1} x_{4}^{2^{s+1}-1}+\beta_{16} x_{1}^{2^{s}-1} x_{3}^{3.2^{s}-1} x_{4}^{2^{s+1}-1}\right) .
\end{aligned}
$$

Using Cartan's formula and Theorem 3.2, we have

$$
\begin{array}{lll}
x_{1}^{3.2^{s}-1} x_{2}^{2^{s+1}-1} x_{4}^{2^{s}-1}=S q^{2}\left(x_{1}^{3.2^{s}-3} x_{2}^{2^{s+1}-1} x_{4}^{2^{s}-1}\right)+a_{s, 23} & \bmod \left(\widehat{A}\left(P_{4}\right)_{n_{s}}\right) \\
x_{1}^{3.2^{s}-1} x_{2}^{2^{s+1}-1} x_{3}^{2^{s}-1}=S q^{2}\left(x_{1}^{3.2^{s}-3} x_{2}^{2^{s+1}-1} x_{3}^{2^{s}-1}\right)+a_{s, 24} & \bmod \left(\widehat{A}\left(P_{4}\right)_{n_{s}}\right) \\
x_{2}^{3.2^{s}-1} x_{3}^{2^{s+1}-1} x_{4}^{2^{s}-1} & =S q^{2}\left(x_{2}^{3.2^{s}-3} x_{3}^{2^{s+1}-1} x_{4}^{2^{s}-1}\right)+a_{s, 15} & \bmod \left(\widehat{A}\left(P_{4}\right)_{n_{s}}\right) \\
x_{1}^{2^{s}-1} x_{2}^{3.2^{s}-1} x_{3}^{2^{s+1}-1} & =S q^{2}\left(x_{1}^{2^{s}-1} x_{2}^{3.2^{s}-3} x_{3}^{2^{s+1}-1}\right)+a_{s, 18} & \bmod \left(\widehat{A}\left(P_{4}\right)_{n_{s}}\right) \\
x_{2}^{2^{s}-1} x_{3}^{3.2^{s}-1} x_{4}^{2^{s+1}-1} & =S q^{2}\left(x_{2}^{2^{s}-1} x_{3}^{3.2^{s}-3} x_{4}^{2^{s+1}-1}\right)+a_{s, 13} & \bmod \left(\widehat{A}\left(P_{4}\right)_{n_{s}}\right) \\
x_{1}^{2^{s}-1} x_{3}^{3.2^{s}-1} x_{4}^{2^{s+1}-1} & =S q^{2}\left(x_{1}^{2^{s}-1} x_{3}^{3.2^{s}-3} x_{4}^{2^{s+1}-1}\right)+a_{s, 16} & \bmod \left(\widehat{A}\left(P_{4}\right)_{n_{s}}\right)
\end{array}
$$

Then, using the equalities (15), (16) and (17) and the relations $\sigma_{i}(g) \equiv g$, for $i \in\{1,2,3\}$, deduce that $\beta_{13}=\beta_{j}$ for all $j, 14 \leqslant j \leqslant 24$. The lemma is completely proved.

Proof of Theorem 2.1.1. Due to Sum [Su14], the basis for $\overline{\boldsymbol{Q}_{n_{s}}^{4}}$ is a set consisting of all the classes represented by the following monomials:

For $s \geqslant 1$,

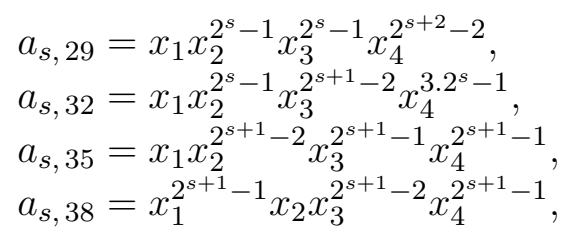

$$
\begin{aligned}
& a_{s, 30}=x_{1} x_{2}^{2^{s}-1} x_{3}^{2^{s+2}-2} x_{4}^{2^{s}-1} \\
& a_{s, 33}=x_{1} x_{2}^{2^{s+1}-2} x_{3}^{2^{s}-1} x_{4}^{3.2^{s}-1} \\
& a_{s, 36}=x_{1} x_{2}^{2^{s+1}-1} x_{3}^{2^{s+1}-2} x_{4}^{2^{s+1}-1} \\
& a_{s, 39}=x_{1}^{2^{s+1}-1} x_{2} x_{3}^{2^{s+1}-1} x_{4}^{2^{s+1}-2}
\end{aligned}
$$

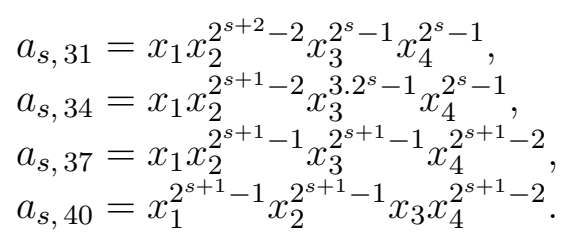

For $s=1$,

$$
\begin{array}{lll}
a_{1,41}=x_{1} x_{2} x_{3}^{3} x_{4}^{4}, & a_{1,42}=x_{1} x_{2}^{3} x_{3} x_{4}^{4}, & a_{1,43}=x_{1} x_{2}^{3} x_{3}^{4} x_{4} \\
a_{1,44}=x_{1}^{3} x_{2} x_{3} x_{4}^{4}, & a_{1,45}=x_{1}^{3} x_{2} x_{3}^{4} x_{4}, & a_{1,46}=x_{1}^{3} x_{2}^{4} x_{3} x_{4}
\end{array}
$$

For $s \geqslant 2$,

$$
\begin{gathered}
a_{s, 41}=x_{1} x_{2}^{2^{s}-2} x_{3}^{2^{s}-1} x_{4}^{2^{s+2}-1} \\
a_{s, 44}=x_{1} x_{2}^{2^{s}-1} x_{3}^{2^{s+2}-1} x_{4}^{2^{s}-2} \\
a_{s, 47}=x_{1}^{2^{s}-1} x_{2} x_{3}^{2^{s}-2} x_{4}^{2^{s+2}-1} \\
a_{s, 50}=x_{1}^{2^{s+2}-1} x_{2} x_{3}^{2^{s}-2} x_{4}^{2^{s}-1} \\
a_{s, 53}=x_{1} x_{2}^{2^{s}-2} x_{3}^{2^{s+1}-1} x_{4}^{3.2^{s}-1} \\
a_{s, 56}=x_{1}^{2^{s+1}-1} x_{2} x_{3}^{2^{s}-2} x_{4}^{3.2^{s}-1} \\
a_{s, 59}=x_{1}^{2^{s}-1} x_{2} x_{3}^{2^{s}-1} x_{4}^{2^{s+2}-2}
\end{gathered}
$$

$$
\begin{aligned}
& a_{s, 43}=x_{1} x_{2}^{2^{s}-1} x_{3}^{2^{s}-2} x_{4}^{2^{s+2}-1}, \\
& a_{s, 46}=x_{1} x_{2}^{2^{s+2}-1} x_{3}^{2^{s}-1} x_{4}^{2^{s}-2}, \\
& a_{s, 49}=x_{1}^{2^{2}-1} x_{2}^{2^{s+2}-1} x_{3} x_{4}^{2^{s}-2}, \\
& a_{s, 52}=x_{1}^{2^{s+2}-1} x_{2}^{2^{s}-1} x_{3} x_{4}^{2^{s}-2}, \\
& a_{s, 55}=x_{1} x_{2}^{2^{s+1}-1} x_{3}^{32^{s}-1} x_{4}^{2^{s}-2}, \\
& a_{s, 58}=x_{1}^{2^{s+1}-1} x_{2}^{3.2^{s}-1} x_{3} x_{4}^{2^{s}-2}, \\
& a_{s, 61}=x_{1}^{2^{s}-1} x_{2}^{2^{s}-1} x_{3} x_{4}^{2^{s+2}-2},
\end{aligned}
$$




$a_{s, 62}=x_{1}^{2^{s}-1} x_{2} x_{3}^{2^{s+1}-2} x_{4}^{3.2^{s}-1}$
$a_{s, 65}=x_{1} x_{2}^{2^{s+1}-1} x_{3}^{3.2^{s}-2} x_{4}^{2^{s}-1}$
$a_{s, 68}=x_{1}^{2^{s+1}-1} x_{2} x_{3}^{2^{s}-1} x_{4}^{3.2^{s}-2}$
$a_{s, 71}=x_{1}^{3} x_{2}^{2^{s}-1} x_{3}^{2^{s+2}-3} x_{4}^{2^{s}-2}$
$a_{s, 74}=x_{1}^{3} x_{2}^{2^{s+1}-3} x_{3}^{2^{s}-2} x_{4}^{3.2^{s}-1}$
$a_{s, 77}=x_{1}^{2^{s+1}-1} x_{2}^{3} x_{3}^{3.2^{s}-3} x_{4}^{2^{s}-2}$
$a_{s, 80}=x_{1}^{3} x_{2}^{2^{s+1}-3} x_{3}^{3.2^{s}-2} x_{4}^{2^{s}-1}$
$a_{s, 83}=x_{1}^{3} x_{2}^{2^{s+1}-1} x_{3}^{2^{s+1}-3} x_{4}^{2^{s+1}-2}$

$$
\begin{aligned}
& a_{s, 63}=x_{1} x_{2}^{2^{s}-1} x_{3}^{2^{s+1}-1} x_{4}^{3.2^{s}-2}, \\
& a_{s, 66}=x_{1}^{2^{s}-1} x_{2} x_{3}^{2^{s+1}-1} x_{4}^{3.2^{s}-2} \\
& a_{s, 69}=x_{1}^{2^{s+1}-1} x_{2} x_{3}^{3.2^{s}-2} x_{4}^{2^{s}-1} \\
& a_{s, 72}=x_{1}^{3} x_{2}^{2^{s+2}-3} x_{3}^{2^{s}-2} x_{4}^{2^{s}-1} \\
& a_{s, 75}=x_{1}^{3} x_{2}^{2^{s+1}-3} x_{3}^{3.2^{s}-1} x_{4}^{2^{s}-2} \\
& a_{s, 78}=x_{1}^{3} x_{2}^{2^{s}-1} x_{3}^{2^{s+1}-3} x_{4}^{3.2^{s}-2} \\
& a_{s, 81}=x_{1}^{3} x_{2}^{2^{s+1}-3} x_{3}^{2^{s+1}-2} x_{4}^{2^{s+1}-1}, \\
& a_{s, 84}=x_{1}^{2^{s+1}-1} x_{2}^{3} x_{3}^{2^{s+1}-3} x_{4}^{2^{s+1}-2}
\end{aligned}
$$

$$
\begin{aligned}
& a_{s, 64}=x_{1} x_{2}^{2^{s+1}-1} x_{3}^{2^{s}-1} x_{4}^{3.2^{s}-2}, \\
& a_{s, 67}=x_{1}^{2^{s}-1} x_{2}^{2^{s+1}-1} x_{3} x_{4}^{3.2^{s}-2}, \\
& a_{s, 70}=x_{1}^{2^{s+1}-1} x_{2}^{2^{s}-1} x_{3} x_{4}^{3.2^{s}-2}, \\
& a_{s, 73}=x_{1}^{3} x_{2}^{2^{s+2}-3} x_{3}^{2^{s}-1} x_{4}^{2^{s}-2}, \\
& a_{s, 76}=x_{1}^{3} x_{2}^{2^{s+1}-1} x_{3}^{3.2^{s}-3} x_{4}^{2^{s}-2}, \\
& a_{s, 79}=x_{1}^{3} x_{2}^{2^{s+1}-3} x_{3}^{2^{s}-1} x_{4}^{3.2^{s}-2}, \\
& a_{s, 82}=x_{1}^{3} x_{2}^{2^{s+1}-3} x_{3}^{2^{s+1}-1} x_{4}^{2^{s+1}-2},
\end{aligned}
$$

For $s=2$

$$
\begin{array}{lll}
a_{2,85}=x_{1}^{3} x_{2}^{3} x_{3}^{3} x_{4}^{12}, & a_{2,86}=x_{1}^{3} x_{2}^{3} x_{3}^{12} x_{4}^{3}, & a_{2,87}=x_{1}^{7} x_{2}^{9} x_{3}^{2} x_{4}^{3}, \\
a_{2,88}=x_{1}^{7} x_{2}^{9} x_{3}^{3} x_{4}^{2}, & a_{2,89}=x_{1}^{3} x_{2}^{3} x_{3}^{4} x_{4}^{11}, & a_{2,90}=x_{1}^{3} x_{2}^{3} x_{3}^{7} x_{4}^{8}, \\
a_{2,91}=x_{1}^{3} x_{2}^{7} x_{3}^{3} x_{4}^{8}, & a_{2,92}=x_{1}^{3} x_{2}^{7} x_{3}^{8} x_{4}^{3}, & a_{2,93}=x_{1}^{7} x_{2}^{3} x_{3}^{3} x_{4}^{8}, \\
a_{2,94}=x_{1}^{7} x_{2}^{3} x_{3}^{8} x_{4}^{3} . & &
\end{array}
$$

For $s \geqslant 3$,

$$
\begin{aligned}
& a_{s, 85}=x_{1}^{3} x_{2}^{2^{s}-3} x_{3}^{2^{s}-2} x_{4}^{2^{s+2}-1}, \\
& a_{s, 88}=x_{1}^{2^{s+2}-1} x_{2}^{3} x_{3}^{2^{s}-3} x_{4}^{2^{s}-2}, \\
& a_{s, 91}=x_{1}^{3} x_{2}^{2^{s}-1} x_{3}^{2^{s}-3} x_{4}^{2^{s+2}-2}, \\
& a_{s, 94}=x_{1}^{3} x_{2}^{2^{s}-3} x_{3}^{2^{s+1}-2} x_{4}^{3.2^{s}-1}, \\
& a_{s, 97}=x_{1}^{2^{s+1}-1} x_{2}^{3} x_{3}^{2^{s}-3} x_{4}^{3.2^{s}-2}, \\
& a_{s, 100}=x_{1}^{7} x_{2}^{2^{s+1}-5} x_{3}^{2^{s}-3} x_{4}^{3.2^{s}-2},
\end{aligned}
$$

$$
\begin{gathered}
a_{s, 86}=x_{1}^{3} x_{2}^{2^{s}-3} x_{3}^{2^{s+2}-1} x_{4}^{2^{s}-2}, \\
a_{s, 89}=x_{1}^{3} x_{2}^{2^{s}-3} x_{3}^{2^{s}-1} x_{4}^{2^{s+2}-2}, \\
a_{s, 92}=x_{1}^{2^{s}-1} x_{2}^{3} x_{3}^{2^{s}-3} x_{4}^{2^{s+2}-2}, \\
a_{s, 95}=x_{1}^{3} x_{2}^{2^{s}-3} x_{3}^{2^{s+1}-1} x_{4}^{3.2^{s}-2}, \\
a_{s, 98}=x_{1}^{2^{s}-1} x_{2}^{3} x_{3}^{2^{s+1}-3} x_{4}^{3.2^{s}-2}, \\
a_{s, 101}=x_{1}^{7} x_{2}^{2^{s+1}-5} x_{3}^{3.2^{s}-3} x_{4}^{2^{s}-2},
\end{gathered}
$$

$$
\begin{aligned}
& a_{s, 87}=x_{1}^{3} x_{2}^{2^{s+2}-1} x_{3}^{2^{s}-3} x_{4}^{2^{s}-2}, \\
& a_{s, 90}=x_{1}^{3} x_{2}^{2^{s}-3} x_{3}^{2^{s+2}-2} x_{4}^{2^{s}-1}, \\
& a_{s, 93}=x_{1}^{2^{s}-1} x_{2}^{3} x_{3}^{2^{s+2}-3} x_{4}^{2^{s}-2}, \\
& a_{s, 96}=x_{1}^{3} x_{2}^{2^{s+1}-1} x_{3}^{2^{s}-3} x_{4}^{3.2^{s}-2}, \\
& a_{s, 99}=x_{1}^{7} x_{2}^{2^{s+2}-5} x_{3}^{2^{s}-3} x_{4}^{2^{s}-2}, \\
& a_{s, 102}=x_{1}^{7} x_{2}^{2^{s+1}-5} x_{3}^{2^{s+1}-3} x_{4}^{2^{s+1}-2} .
\end{aligned}
$$

For $s=3$,

$$
a_{3,103}=x_{1}^{7} x_{2}^{7} x_{3}^{7} x_{4}^{24}, \quad a_{3,104}=x_{1}^{7} x_{2}^{7} x_{3}^{9} x_{4}^{22}, \quad a_{3,105}=x_{1}^{7} x_{2}^{7} x_{3}^{25} x_{4}^{6}
$$

For $s \geqslant 4$,

$$
a_{s, 103}=x_{1}^{7} x_{2}^{2^{s}-5} x_{3}^{2^{s}-3} x_{4}^{2^{s+2}-2}, \quad a_{s, 104}=x_{1}^{7} x_{2}^{2^{s}-5} x_{3}^{2^{s+1}-3} x_{4}^{3.2^{s}-2}, \quad a_{s, 105}=x_{1}^{7} x_{2}^{2^{s}-5} x_{3}^{2^{s+2}-3} x_{4}^{2^{s}-2} \text {. }
$$

We now consider the following cases.

Case $s=1$. We have a direct summand decomposition of the $\Sigma_{4}$-modules:

$$
\overline{Q_{n_{1}}^{4}}=\Sigma_{4}\left(a_{1,29}, a_{1,32}\right) \bigoplus \Sigma_{4}\left(a_{1,35}\right),
$$

wherein $\Sigma_{4}\left(a_{1,29} ; a_{1,32}\right)=\left\langle\left\{\left[a_{1, j}\right]: j=29,30, \ldots, 34,41,42, \ldots, 46\right\}\right\rangle$, and

$$
\Sigma_{4}\left(a_{1,35}\right)=\left\langle\left\{\left[a_{1, j}\right]: 35 \leqslant j \leqslant 40\right\}\right\rangle .
$$

Lemma 3.1.2. The following statements are true:

i) $\Sigma_{4}\left(a_{1,29} ; a_{1,32}\right)^{\Sigma_{4}}=\left\langle\left[q_{1,4}\right]\right\rangle$, with $q_{1,4}=\sum_{29 \leqslant j \leqslant 31} a_{1, j}+\sum_{44 \leqslant j \leqslant 46} a_{1, j}$;

ii) $\Sigma_{4}\left(a_{1,35}\right)^{\Sigma_{4}}=0$.

Combining Lemmas 3.1.1 and 3.1.2 gives

Corollary 3.1.3. The space of $\Sigma_{4}$-invariants $\left(Q_{n_{1}}^{4}\right)^{\Sigma_{4}}$ is generated by the classes $\left[q_{1, t}\right]$ for all $t, 1 \leqslant t \leqslant 4$.

Proof of Lemma 3.1.2. The proof of $i$ ) is straightforward. We prove $i$ ) in detail. Suppose that $[g] \in \Sigma_{4}\left(a_{1,29} ; a_{1,32}\right)^{\Sigma_{4}}$. Then, we have

$$
g \equiv\left(\sum_{29 \leqslant j \leqslant 34} \gamma_{j} a_{1, j}+\sum_{41 \leqslant j \leqslant 46} \gamma_{j} a_{1, j}\right),
$$


with $\gamma_{j} \in k$. Acting the homomorphisms $\sigma_{i}: P_{4} \longrightarrow P_{4}, 1 \leqslant i \leqslant 3$, on both sides of (18), we get

$$
\begin{aligned}
\sigma_{1}(g) \equiv & \left(\gamma_{29} a_{1,29}+\gamma_{30} a_{1,30}+\gamma_{32} a_{1,32}+\gamma_{41} a_{1,41}+\gamma_{44} a_{1,42}\right. \\
& +\gamma_{45} a_{1,43}+\gamma_{42} a_{1,44}+\gamma_{43} a_{1,45}+\gamma_{31} x_{1}^{6} x_{2} x_{3} x_{4} \\
& \left.+\gamma_{33} x_{1}^{2} x_{2} x_{3} x_{4}^{5}+\gamma_{34} x_{1}^{2} x_{2} x_{3}^{5} x_{4}+\gamma_{46} x_{1}^{4} x_{2}^{3} x_{3} x_{4}\right) \\
\sigma_{2}(g) \equiv & \left(\gamma_{29} a_{1,29}+\gamma_{31} a_{1,30}+\gamma_{30} a_{1,31}+\gamma_{33} a_{1,32}+\gamma_{42} a_{1,41}+\gamma_{41} a_{1,42}\right. \\
& \left.+\gamma_{44} a_{1,44}+\gamma_{46} a_{1,45}+\gamma_{45} a_{1,46}+\gamma_{34} x_{1} x_{2}^{5} x_{3}^{2} x_{4}+\gamma_{43} x_{1} x_{2}^{4} x_{3}^{3} x_{4}\right) \\
\sigma_{3}(g) \equiv & \left(\gamma_{30} a_{1,29}+\gamma_{29} a_{1,30}+\gamma_{31} a_{1,31}+\gamma_{34} a_{1,43}+\gamma_{33} a_{1,34}+\gamma_{43} a_{1,42}+\gamma_{42} a_{1,43}\right. \\
& \left.+\gamma_{45} a_{1,44}+\gamma_{44} a_{1,45}+\gamma_{46} a_{1,46}+\gamma_{32} x_{1} x_{2} x_{3}^{5} x_{4}^{2}+\gamma_{41} x_{1} x_{2} x_{3}^{4} x_{4}^{3}\right) .
\end{aligned}
$$

Using the Cartan formula and Theorem 3.2, we have

$$
\begin{aligned}
x_{1}^{6} x_{2} x_{3} x_{4}= & S q^{1}\left(x_{1}^{5} x_{2} x_{3} x_{4}\right)+S q^{2}\left(x_{1}^{3} x_{2}^{2} x_{3} x_{4}+x_{1}^{3} x_{2} x_{3}^{2} x_{4}+x_{1}^{3} x_{2} x_{3} x_{4}^{2}\right) \\
& +a_{1,44}+a_{1,45}+a_{1,46} \bmod \left(\widehat{A}\left(P_{4}\right)_{n_{1}}\right) ; \\
x_{1}^{2} x_{2} x_{3} x_{4}^{5}= & S q^{1}\left(x_{1} x_{2} x_{3} x_{4}^{5}\right)+a_{1,29}+a_{1,32}+a_{1,33} \quad \bmod \left(\widehat{A}\left(P_{4}\right)_{n_{1}}\right) ; \\
x_{1}^{2} x_{2} x_{3}^{5} x_{4}= & S q^{1}\left(x_{1} x_{2} x_{3}^{5} x_{4}\right)+S q^{2}\left(x_{1} x_{2} x_{3}^{3} x_{4}^{2}\right)+a_{1,30}+a_{1,34}+a_{1,41} \quad \bmod \left(\widehat{A}\left(P_{4}\right)_{n_{1}}\right) ; \\
x_{1}^{4} x_{2}^{3} x_{3} x_{4}= & S q^{1}\left(x_{1} x_{2}^{5} x_{3} x_{4}\right)+S q^{2}\left(x_{1} x_{2}^{3} x_{3} x_{4}^{2}+x_{1} x_{2}^{3} x_{3}^{2} x_{4}+x_{1}^{2} x_{2}^{3} x_{3} x_{4}\right) \\
& +a_{1,31}+a_{1,42}+a_{1,43} \bmod \left(\widehat{A}\left(P_{4}\right)_{n_{1}}\right) ; \\
x_{1} x_{2}^{5} x_{3}^{2} x_{4}= & S q^{2}\left(x_{1} x_{2}^{3} x_{3}^{2} x_{4}\right)+a_{1,43} \bmod \left(\widehat{A}\left(P_{4}\right)_{n_{1}}\right) ; \\
x_{1} x_{2}^{4} x_{3}^{3} x_{4}= & S q^{2}\left(x_{1} x_{2}^{2} x_{3}^{3} x_{4}\right)+a_{1,34} \bmod \left(\widehat{A}\left(P_{4}\right)_{n_{1}}\right) ; \\
x_{1} x_{2} x_{3}^{5} x_{4}^{2}= & S q^{2}\left(x_{1} x_{2} x_{3}^{3} x_{4}^{2}\right)+a_{1,41} \bmod \left(\widehat{A}\left(P_{4}\right)_{n_{1}}\right) ; \\
x_{1} x_{2} x_{3}^{4} x_{4}^{3}= & S q^{2}\left(x_{1} x_{2} x_{3}^{2} x_{4}^{3}\right)+a_{1,32} \bmod \left(\widehat{A}\left(P_{4}\right)_{n_{1}}\right) .
\end{aligned}
$$

Combining (18), (19) and (20), we get

$$
\begin{aligned}
\left(\sigma_{1}(g)+g\right) \equiv & \left(\gamma_{33}\left(a_{1,29}+a_{1,32}\right)+\gamma_{34}\left(a_{1,30}+a_{1,41}\right)+\left(\gamma_{31}+\gamma_{46}\right) a_{1,31}+\left(\gamma_{42}+\gamma_{44}+\gamma_{46}\right) a_{1,42}\right. \\
& \left.+\left(\gamma_{43}+\gamma_{45}+\gamma_{46}\right) a_{1,43}+\left(\gamma_{31}+\gamma_{42}+\gamma_{44}\right) a_{1,44}+\left(\gamma_{31}+\gamma_{43}+\gamma_{45}\right) a_{1,45}\right), \\
\left(\sigma_{2}(g)+g\right) \equiv & \left(\left(\gamma_{30}+\gamma_{31}\right) a_{1,30}+\left(\gamma_{32}+\gamma_{33}\right) a_{1,32}+\left(\gamma_{34}+\gamma_{43}\right) a_{1,34}\right. \\
& \left.+\left(\gamma_{41}+\gamma_{42}\right) a_{1,41}+\left(\gamma_{45}+\gamma_{46}\right) a_{1,45}\right) \\
\left(\sigma_{3}(g)+g\right) \equiv & \left(\left(\gamma_{29}+\gamma_{30}\right) a_{1,29}+\left(\gamma_{32}+\gamma_{41}\right) a_{1,32}+\left(\gamma_{33}+\gamma_{34}\right) a_{1,33}\right. \\
& \left.+\left(\gamma_{42}+\gamma_{43}\right) a_{1,42}+\left(\gamma_{44}+\gamma_{45}\right) a_{1,44}\right) .
\end{aligned}
$$

From these equalities and the relations $\sigma_{j}(g) \equiv g, 1 \leqslant j \leqslant 3$, we obtain $\gamma_{j}=0$ for $j \in$ $\{32,33,34,41,42,43\}$, and $\gamma_{29}=\gamma_{30}=\gamma_{31}=\gamma_{44}=\gamma_{45}=\gamma_{46}$. This completes the proof of the lemma.

The following next assertion is useful.

Proposition 3.1.4. We have $\left(\boldsymbol{Q}_{n_{1}}^{4}\right)^{G(4)}=\left\langle\left[q_{1,4}\right]\right\rangle$.

Proof. Let $h \in\left(P_{4}\right)_{n_{1}}$ such that $[h] \in\left(\boldsymbol{Q}_{n_{1}}^{4}\right)^{G(4)}$. Since $\Sigma_{4} \subset G(4),[h] \in\left(\boldsymbol{Q}_{n_{1}}^{4}\right)^{\Sigma_{4}}$ and therefore by Corollary 3.1.3, we have $h \equiv \sum_{1 \leqslant t \leqslant 4} m_{t} q_{1, t}$, wherein $m_{t} \in k$ for every $t$. Since $[h] \in\left(\boldsymbol{Q}_{n_{1}}^{4}\right)^{G(4)}$, $\left(\sigma_{4}(h)+h\right) \equiv 0$ with $\sigma_{4}: P_{4} \longrightarrow P_{4}$. From Theorem 3.2 and a direct computation, we obtain

$$
\left(\sigma_{4}(h)+h\right) \equiv\left(m_{1} a_{1,1}+\left(m_{1}+m_{2}\right) a_{1,8}+\left(m_{2}+m_{3}\right) a_{1,17}+\text { other terms }\right) \equiv 0 .
$$

and therefore $h \equiv q_{1,4}$. The proposition is proved.

Now, we consider the element

$$
\zeta=\left(a_{1}^{(1)} a_{2}^{(3)} a_{3}^{(3)} a_{4}^{(2)}+a_{1}^{(1)} a_{2}^{(3)} a_{3}^{(4)} a_{4}^{(1)}+a_{1}^{(1)} a_{2}^{(5)} a_{3}^{(2)} a_{4}^{(1)}+a_{1}^{(1)} a_{2}^{(6)} a_{3}^{(1)} a_{4}^{(1)}\right) \in\left(P_{4}\right)_{3\left(2^{1}-1\right)+3.2^{1}}^{*}
$$


Then, it is $\widehat{A}$-annihilated. Indeed, by the unstable condition, we need only to consider the effects of $S q^{1}$ and $S q^{2}$. By a direct computation, we get

$$
\begin{aligned}
& (\zeta) S q^{1}=a_{1}^{(1)} a_{2}^{(3)} a_{3}^{(3)} a_{4}^{(1)}+a_{1}^{(1)} a_{2}^{(3)} a_{3}^{(3)} a_{4}^{(1)}+a_{1}^{(1)} a_{2}^{(5)} a_{3}^{(1)} a_{4}^{(1)}+a_{1}^{(1)} a_{2}^{(5)} a_{3}^{(1)} a_{4}^{(1)}=0, \\
& (\zeta) S q^{2}=0+a_{1}^{(1)} a_{2}^{(3)} a_{3}^{(2)} a_{4}^{(1)}+a_{1}^{(1)} a_{2}^{(3)} a_{3}^{(2)} a_{4}^{(1)}+0=0 .
\end{aligned}
$$

On the other side, since $\left\langle\zeta, q_{1,4}>=1\right.$, by Proposition 3.1.4, implies that

$$
k \otimes_{G(4)} P_{A}\left(\left(P_{4}\right)_{n_{1}}^{*}\right)=\left(\left(\boldsymbol{Q}_{n_{1}}^{4}\right)^{G(4)}\right)^{*}=\left\langle\left(\left[q_{1,4}\right]\right)^{*}\right\rangle=\langle[\zeta]\rangle .
$$

Case $s=2$. We first have the following remark.

Remark 3.1.5. Based on the bases of $\boldsymbol{Q}_{n_{s}}^{4}$ and $\overline{\boldsymbol{Q}_{n_{s}}^{4}}$ above, the weight vector of $a_{s, j}$ is one of the following sequences:

$$
\omega_{(s, 1)}:=\underbrace{(3,3, \ldots, 3}_{s \text { times of } 3}, 1,1), \quad \omega_{(s, 2)}:=\underbrace{(3,3, \ldots, 3)}_{(s+1) \text { times of } 3} .
$$

Hence, we have the following isomorphisms:

$$
\begin{array}{ll}
\boldsymbol{Q}_{n_{s}}^{4} & \cong\left(\boldsymbol{Q}_{n_{s}}^{4}\right)^{\omega_{(s, 1)}} \oplus\left(\boldsymbol{Q}_{n_{s}}^{4}\right)^{\omega_{(s, 2)}}, \\
\left(\boldsymbol{Q}_{n_{s}}^{4}\right)^{\omega_{(s, j)}} \cong\left({\underline{\boldsymbol{Q}_{n_{s}}^{4}}}^{4}\right)^{\omega_{(s, j)}} \bigoplus\left(\overline{\boldsymbol{Q}_{n_{s}}^{4}}\right)^{\omega_{(s, j)}}, j=1,2 .
\end{array}
$$

Then, for $s=2$, we have $\boldsymbol{Q}_{n_{2}}^{4} \cong\left(\boldsymbol{Q}_{n_{2}}^{4}\right)^{\omega_{(2,1)}} \bigoplus\left(\boldsymbol{Q}_{n_{2}}^{4}\right)^{\omega_{(2,2)}}$ and the following.

Proposition 3.1.6. The spaces of invariants $\left(\left(\boldsymbol{Q}_{n_{2}}^{4}\right)^{\omega_{(2,1)}}\right)^{G(4)}$ and $\left(\left(\boldsymbol{Q}_{n_{2}}^{4}\right)^{\omega_{(2,2)}}\right)^{G(4)}$ are trivial.

Proof. We first prove $\left(\left(\boldsymbol{Q}_{n_{2}}^{4}\right)^{\omega_{(2,1)}}\right)^{G(4)}=0$. It is clear that the monomial $a_{s, 12}=x_{1}^{2^{s+2}-1} x_{2}^{2^{s}-1} x_{3}^{2^{s}-1}$ is minimal spike in $\left(P_{4}\right)_{n_{s}}$ and $\omega\left(a_{s, 12}\right)=\omega_{(s, 1)}$ for any $s \geqslant 1$. Hence, $\left[a_{s, j}\right]_{\omega_{(s, 1)}}=\left[a_{s, j}\right]$. Recall that $\left(\boldsymbol{Q}_{n_{2}}^{4}\right)^{\omega_{(2,1)}} \cong\left({\underline{\boldsymbol{Q}_{n_{2}}}}^{4}\right)^{\omega_{(2,1)}} \bigoplus\left(\overline{\boldsymbol{Q}_{n_{2}}^{4}}\right)^{\omega_{(2,1)}}$. Then, by Lemma 3.1.1, we get $\left(\underline{\boldsymbol{Q}_{n_{2}}^{4}}\right)^{\omega_{(2,1)}}=\Sigma_{4}\left(a_{2,1}\right) \bigoplus \Sigma_{4}\left(a_{2,13}\right)$, and

$$
\left(\left(\underline{\boldsymbol{Q}_{n_{2}}^{4}}\right)^{\omega_{(2,1)}}\right)^{\Sigma_{4}}=\left\langle\left[q_{2,1}\right],\left[q_{2,2}\right]\right\rangle .
$$

Using an admissible basis of $\overline{\boldsymbol{Q}_{n_{2}}^{4}}$ above, we have a direct summand decomposition of the $\Sigma_{4^{-}}$ modules:

$$
\left(\overline{\boldsymbol{Q}_{n_{2}}^{4}}\right)^{\omega_{(2,1)}}=\Sigma_{4}\left(a_{2,41}\right) \bigoplus \Sigma_{4}\left(a_{2,53}\right) \bigoplus \mathbb{V}
$$

wherein

$$
\begin{aligned}
& \Sigma_{4}\left(a_{2,41}\right)=\left\langle\left\{\left[a_{2, j}\right]: 41 \leqslant j \leqslant 52\right\}\right\rangle, \Sigma_{4}\left(a_{2,53}\right)=\left\langle\left\{\left[a_{2, j}\right]: 53 \leqslant j \leqslant 58\right\}\right\rangle, \\
& \mathbb{V}=\left\langle\left\{\left[a_{2, j}\right]: j=29, \ldots, 34,59, \ldots, 80,85, \ldots, 94\right\}\right\rangle .
\end{aligned}
$$

Then, by a similar technique as in the proof of Lemma 3.1.2, we find that

$$
\begin{aligned}
& \Sigma_{4}\left(a_{2,41}\right)^{\Sigma_{4}}=\left\langle\left[\widehat{q_{2,1}}\right]\right\rangle \text { with } \widehat{q_{2,1}}:=\sum_{41 \leqslant j \leqslant 52} a_{2, j} ; \\
& \Sigma_{4}\left(a_{2,53}\right)^{\Sigma_{4}}=\left\langle\left[\widehat{q_{2,2}}\right]\right\rangle \text { with } \widehat{q_{2,2}}:=\sum_{53 \leqslant j \leqslant 58} a_{2, j} ; \\
& \mathbb{V}^{\Sigma_{4}}=0 .
\end{aligned}
$$

Thus, from the equalities (21) and (22), we get

$$
\left(\left(\boldsymbol{Q}_{n_{2}}^{4}\right)^{\omega_{(2,1)}}\right)^{\Sigma_{4}}=\left\langle\left[q_{2,1}\right],\left[q_{2,2}\right],\left[\widehat{q_{2,1}}\right],\left[\widehat{q_{2,2}}\right]\right\rangle .
$$

Now, let $h \in\left(P_{4}\right)_{n_{2}}$ such that $[h] \in\left(\left(\boldsymbol{Q}_{n_{2}}^{4}\right)^{\omega_{(2,1)}}\right)^{G(4)}$. Since $[h] \in\left(\left(\boldsymbol{Q}_{n_{2}}^{4}\right)^{\omega_{(2,1)}}\right)^{\Sigma_{4}}$, by the equality (23), we have

$$
h \equiv\left(\xi_{1} q_{2,1}+\xi_{2} q_{2,2}+\xi_{3} \widehat{q_{2,1}}+\xi_{4} \widehat{q_{2,2}}\right),
$$

wherein $\xi_{t} \in k$ for $1 \leqslant t \leqslant 4$. Acting the homomorphism $\sigma_{4}: P_{4} \longrightarrow P_{4}$ on both sides of (24), and using Theorem 3.2 with the relation $\left(\sigma_{4}(h)+h\right) \equiv 0$, we obtain

$$
\left(\sigma_{4}(h)+h\right) \equiv\left(\left(\xi_{1}+\xi_{3}\right) a_{2,1}+\xi_{1} a_{2,3}+\left(\xi_{1}+\xi_{2}\right) a_{2,8}+\left(\xi_{2}+\xi_{4}\right) a_{2,13}+\text { other terms }\right) \equiv 0 .
$$


The last equality implies $\xi_{1}=\xi_{2}=\xi_{3}=\xi_{4}$. This means that $\left(\left(\boldsymbol{Q}_{n_{2}}^{4}\right)^{\omega_{(2,1)}}\right)^{G(4)}=0$.

Next, we have $\left(\boldsymbol{Q}_{n_{2}}^{4}\right)^{\omega_{(2,2)}} \cong\left({\underline{\boldsymbol{Q}_{n_{2}}}}^{4}\right)^{\omega_{(2,2)}} \bigoplus\left(\overline{\boldsymbol{Q}_{n_{2}}^{4}}\right)^{\omega_{(2,2)}}$. According to Lemma 3.1.1, we get

$$
\left(\left(\underline{\boldsymbol{Q}_{n_{2}}^{4}}\right)^{\omega_{(2,2)}}\right)^{\Sigma_{4}}=\left\langle\left[q_{2,3}\right]_{\omega_{(2,2)}}\right\rangle .
$$

A basis of $\left(\overline{\boldsymbol{Q}_{n_{2}}^{4}}\right)^{\omega_{(2,2)}}$ is the set $\left\{\left[a_{2, j}\right]_{\omega_{(2,2)}}: j=35,36, \ldots, 40,81, \ldots, 84\right\}$ and we have a direct summand decomposition of the $\Sigma_{4}$-modules:

$$
\left(\overline{\boldsymbol{Q}_{n_{2}}^{4}}\right)^{\omega}{ }_{(2,2)}=\Sigma_{4}\left(a_{2,35}\right) \bigoplus \Sigma_{4}\left(a_{2,81}\right)
$$

wherein $\Sigma_{4}\left(a_{2,35}\right)=\left\langle\left\{\left[\left[a_{2, j}\right]_{\omega_{(2,2)}}: j=35, \ldots, 40\right]\right\}\right\rangle$ and $\Sigma_{4}\left(a_{2,81}\right)=\left\langle\left\{\left[\left[a_{2, j}\right]_{\omega_{(2,2)}}: j=81, \ldots, 84\right]\right\}\right\rangle$. By using Theorem 3.2 and a similar computation as above, we also obtain

$$
\Sigma_{4}\left(a_{2,35}\right)^{\Sigma_{4}}=\left\langle\left[\widehat{q_{2,3}}\right]_{\omega_{(2,2)}}\right\rangle, \quad \Sigma_{4}\left(a_{2,81}\right)^{\Sigma_{4}}=\left\langle\left[\widehat{q_{2,4}}\right]_{\omega_{(2,2)}}\right\rangle
$$

wherein $\widehat{q_{2,3}}=\sum_{35 \leqslant j \leqslant 40} a_{2, j}$ and $\widehat{q_{2,4}}=\sum_{81 \leqslant j \leqslant 84} a_{2, j}$. Then,

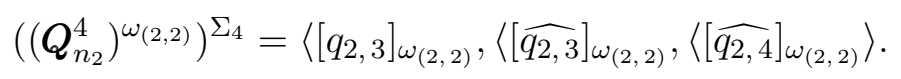

Let $[g]_{\omega_{(2,2)}} \in\left(\left(\boldsymbol{Q}_{n_{2}}^{4}\right)^{\omega_{(2,2)}}\right)^{G(4)}$, we have $g \equiv_{\omega_{(2,2)}}\left(\ell_{1} q_{2,3}+\ell_{2} \widehat{q_{2,3}}+\ell_{3} \widehat{q_{2,4}}\right)$ with $\ell_{i} \in k$ for every $i$. By a direct computation using the relation $\left(\sigma_{4}(g)+g\right) \equiv_{\omega_{(2,2)}} 0$, we obtain

$$
\left(\sigma_{4}(g)+g\right) \equiv_{\omega_{(2,2)}}\left(\left(\ell_{1}+\ell_{2}\right) a_{2,25}+\left(\ell_{2}+\ell_{3}\right) a_{2,36}+\ell_{3} a_{2,83}+\text { other terms }\right) \equiv_{\omega_{(2,2)}} 0 .
$$

This means that $\ell_{1}=\ell_{2}=\ell_{3}=0$. The proposition follows.

Now, since $\operatorname{dim}\left(\boldsymbol{Q}_{n_{2}}^{4}\right)^{G(4)} \leqslant \operatorname{dim}\left(\left(\boldsymbol{Q}_{n_{2}}^{4}\right)^{\omega_{(2,1)}}\right)^{G(4)}+\operatorname{dim}\left(\left(\boldsymbol{Q}_{n_{2}}^{4}\right)^{\omega_{(2,2)}}\right)^{G(4)}$, by Proposition 3.1.6, we get

$$
k \otimes_{G(4)} P_{A}\left(\left(P_{4}\right)_{n_{2}}^{*}\right)=\left(\left(\boldsymbol{Q}_{n_{2}}^{4}\right)^{G(4)}\right)^{*}=0 .
$$

Case $s \geqslant 3$. For simplicity, we prove the cases $s \geqslant 4$. Calculations of the case $s=3$ use similar techniques. Recall that

$$
\boldsymbol{Q}_{n_{s}}^{4} \cong\left(\boldsymbol{Q}_{n_{s}}^{4}\right)^{\omega_{(s, 1)}} \bigoplus\left(\boldsymbol{Q}_{n_{s}}^{4}\right)^{\omega_{(s, 2)}}
$$

wherein $\operatorname{dim}\left(\boldsymbol{Q}_{n_{s}}^{4}\right)^{\omega_{(s, 1)}}=90$ and $\operatorname{dim}\left(\boldsymbol{Q}_{n_{s}}^{4}\right)^{\omega_{(s, 2)}}=15$ for all $s \geqslant 4$.

Proposition 3.1.7. The following hold:

i) $\left(\left(\boldsymbol{Q}_{n_{s}}^{4}\right)^{\omega_{(s, 1)}}\right)^{G(4)}$ is trivial.

ii) $\left(\left(\boldsymbol{Q}_{n_{s}}^{4}\right)^{\omega_{(s, 2)}}\right)^{G(4)}=\left\langle\left[\zeta_{s}^{*}\right]_{\omega_{(s, 2)}}\right\rangle$, wherein

$$
\zeta_{s}^{*}=\sum_{25 \leqslant j \leqslant 28} a_{s, j}+\sum_{35 \leqslant j \leqslant 40} a_{s, j}+\sum_{81 \leqslant j \leqslant 84} a_{s, j}+a_{s, 102}
$$

Outline of the proof. We will give the sketch of proof of ii). The proofs of i) use similar idea.

We have an isomorphism of the $k$-vector spaces

$$
\left(\boldsymbol{Q}_{n_{s}}^{4}\right)^{\omega_{(s, 2)}} \cong\left({\underline{\boldsymbol{Q}_{n_{s}}^{4}}}^{\omega_{(s, 2)}} \bigoplus\left(\overline{\boldsymbol{Q}_{n_{s}}^{4}}\right)^{\omega_{(s, 2)}},\right.
$$

wherein

$$
\begin{aligned}
& \left(\underline{\boldsymbol{Q}_{n_{s}}^{4}}\right)^{\omega_{(s, 2)}}=\left\langle\left\{\left[a_{s, j}\right]_{\omega_{(s, 2)}}: 25 \leqslant j \leqslant 28\right\}\right\rangle, \\
& \left(\overline{\boldsymbol{Q}_{n_{s}}^{4}}\right)^{\omega_{(s, 2)}}=\left\langle\left\{\left[a_{s, j}\right]_{\omega_{(s, 2)}}: j=35, \ldots, 40,81, \ldots, 84,102\right\}\right\rangle .
\end{aligned}
$$

Then, by Lemma 3.1.1,

$$
\left(\left(\underline{\boldsymbol{Q}_{n_{s}}^{4}}\right)^{\omega_{(s, 2)}}\right)^{\Sigma_{4}}=\left\langle\left[q_{s, 3}\right]_{\omega_{(s, 2)}}\right\rangle .
$$

Now, if $[f] \in\left(\left(\overline{\boldsymbol{Q}_{n_{s}}^{4}}\right)^{\omega_{(s, 2)}}\right)^{\Sigma_{4}}$, then we have

$$
f \equiv_{\omega_{(s, 2)}}\left(\sum_{35 \leqslant j \leqslant 40} \gamma_{j} a_{s, j}+\sum_{81 \leqslant j \leqslant 84} \gamma_{j} a_{s, j}+\gamma_{102} a_{s, 102}\right)
$$

in which $\gamma_{j} \in k$. So, by a simple computation using the relations $\sigma_{i}(f) \equiv_{\omega_{(s, 2)}} f$ for $i \in\{1,2,3\}$, wherein $\sigma_{i}: P_{4} \longrightarrow P_{4}$, we get $\gamma_{35}=\gamma_{36}=\cdots=\gamma_{40}$, and $\gamma_{81}=\gamma_{82}=\gamma_{83}=\gamma_{84}$. This leads to

$$
\left(\left(\overline{\boldsymbol{Q}_{n_{s}}^{4}}\right)^{\omega_{(s, 2)}}\right)^{\Sigma_{4}}=\left\langle\left[q_{s, 4}\right]_{\omega_{(s, 2)}},\left[q_{s, 5}\right]_{\omega_{(s, 5)}},\left[a_{s, 102}\right]_{\omega_{(s, 2)}}\right\rangle,
$$


with $q_{s, 4}:=\sum_{35 \leqslant j \leqslant 40} a_{s, j}$ and $q_{s, 5}:=\sum_{81 \leqslant j \leqslant 84} a_{s, j}$.

Let $h \in\left(P_{4}\right)_{n_{s}}$ such that $[h] \in\left(\left(\boldsymbol{Q}_{n_{s}}^{4}\right)^{\omega_{(s, 2)}}\right)^{G(4)}$. Since $\Sigma_{4} \subset G(4)$, from the above calculations, we have

$$
h \equiv_{\omega_{(s, 2)}}\left(\beta_{1} q_{s, 3}+\beta_{2} q_{s, 4}+\beta_{3} q_{s, 5}+\beta_{4} a_{s, 102}\right) \text {, }
$$

with $\beta_{i} \in k$ for every $i$. Direct computing from the relation $\left(\sigma_{4}(h)+h\right) \equiv 0$, we obtain

$$
\left(\sigma_{4}(h)+h\right) \equiv_{\omega_{(s, 2)}}\left(\left(\beta_{1}+\beta_{2}\right) a_{s, 25}+\left(\beta_{2}+\beta_{3}\right)\left(a_{s, 36}+a_{s, 37}\right)+\left(\beta_{3}+\beta_{4}\right) a_{s, 83}\right) \equiv 0,
$$

and this is apparently that $\beta_{1}=\beta_{2}=\beta_{3}=\beta_{4}$. The proposition is proved.

The following inequality is immediate from Proposition 3.1.7.

$$
\operatorname{dim} k \otimes_{G(4)} P_{A}\left(\left(P_{4}\right)_{n_{s}}^{*}\right) \leqslant \operatorname{dim}\left(\left(\boldsymbol{Q}_{n_{s}}^{4}\right)^{\omega_{(s, 1)}}\right)^{G(4)}+\operatorname{dim}\left(\left(\boldsymbol{Q}_{n_{s}}^{4}\right)^{\omega_{(s, s)}}\right)^{G(4)}=1 .
$$

On the other hand, as indicated in the introduction, we can verify that the element

$$
\zeta_{s}=a_{2}^{\left(2^{s+1}-1\right)} a_{3}^{\left(2^{s+1}-1\right)} a_{4}^{\left(2^{s+1}-1\right)} \in\left(P_{4}\right)_{3\left(2^{s}-1\right)+3.2^{s}}^{*} \in P_{A}\left(\left(P_{4}\right)_{n_{s}}^{*}\right)
$$

and that the cycle $\psi_{4}\left(\zeta_{s}\right)=\lambda_{0} \lambda_{s+1}^{3}$ in $\Lambda$ is a representative of $h_{0} h_{s+1}^{3} \in \operatorname{Ext}_{A}^{4,6.2^{s}+1}(k, k)$. Hence, $h_{0} h_{s+1}^{3}$ is in the image of $\operatorname{Tr}_{4}^{A}$. Moreover, by Theorem 2.1, we have $\operatorname{Ext}_{A}^{4,6.2^{s}+1}(k, k)=\left\langle h_{0} h_{s+1}^{3}\right\rangle$ with $h_{0} h_{s+1}^{3}=h_{0} h_{s}^{2} h_{s+2} \neq 0$ for any $s \geqslant 3$. From these data, the coinvariant $k \otimes_{G(4)} P_{A}\left(\left(P_{4}\right)_{n_{s}}^{*}\right)$ is 1-dimensional. So, the theorem follows from the facts that $\left(\boldsymbol{Q}_{n_{s}}^{4}\right)^{G(4)}=\left\langle\left[\zeta_{s}^{*}\right]\right\rangle$ and $\left\langle\zeta_{s}, \zeta_{s}^{*}\right\rangle=1$.

\subsection{Proof of Theorem 2.1.4}

Let $n_{s}:=2^{s+3}+2^{s+1}-3$, in order to prove the theorem, we first have the following.

Proposition 3.2.1. For a positive integer $s$, the invariant spaces $\left(Q_{n_{s}}^{4}\right)^{G(4)}$ are determined as follows:

$$
\left(Q_{n_{s}}^{4}\right)^{G(4)}= \begin{cases}\langle[\widetilde{\zeta}]\rangle & \text { for } s=1, \\ 0 & \text { otherwise },\end{cases}
$$

wherein

$$
\begin{aligned}
\widetilde{\zeta}= & x_{1} x_{2} x_{3} x_{4}^{14}+x_{1} x_{2} x_{3}^{14} x_{4}+x_{1} x_{2}^{3} x_{3} x_{4}^{12}+x_{1} x_{2}^{3} x_{3}^{12} x_{4} \\
& +x_{1}^{3} x_{2} x_{3} x_{4}^{12}+x_{1}^{3} x_{2} x_{3}^{12} x_{4}+x_{1}^{3} x_{2}^{5} x_{3} x_{4}^{8}+x_{1}^{3} x_{2}^{5} x_{3}^{8} x_{4} .
\end{aligned}
$$

Proof. The proof of the proposition is based on an admissible monomial basis of the $k$-vector space $\boldsymbol{Q}_{n_{s}}^{4}$. We have an isomorphism of $k$-vector spaces: $\boldsymbol{Q}_{n_{s}}^{4} \cong \boldsymbol{Q}_{n_{s}}^{4} \oplus \overline{\boldsymbol{Q}_{n_{s}}^{4}}$. According to Sum [Su14], $Q_{n_{s}}^{4}=\left\langle\left\{\left[b_{s, j}\right]: 1 \leqslant j \leqslant 40\right\}\right\rangle$, for all $s \geqslant 1$, wherein the admissible monomials $b_{s, j}$ are determined as follows:

$$
\begin{aligned}
& b_{s, 1}=x_{2}^{2^{s+1}-1} x_{3}^{2^{s+2}-1} x_{4}^{2^{s+2}-1}, \\
& b_{s, 4}=x_{1}^{2^{s+1}-1} x_{3}^{2^{s+2}-1} x_{4}^{2^{s+2}-1}, \\
& b_{s, 7}=x_{1}^{2^{s+1}-1} x_{2}^{2^{s+2}-1} x_{4}^{2^{s+2}-1}, \\
& b_{s, 10}=x_{1}^{2^{s+1}-1} x_{2}^{2^{s+2}-1} x_{3}^{2^{s+2}-1} \text {, } \\
& b_{s, 13}=x_{1}^{2^{s}-1} x_{2}^{2^{s}-1} x_{3}^{2^{s+3}-1} \text {, } \\
& b_{s, 16}=x_{2}^{2^{s}-1} x_{3}^{2^{s+3}-1} \text {, } \\
& b_{s, 19}=x_{1}^{2^{s}-1} x_{3}^{2^{s+3}-1} \text {, } \\
& b_{s, 22}=x_{1}^{2^{s}-1} x_{2}^{2^{s+3}-1} \text {, } \\
& b_{s, 25}=x_{1}^{2^{s}-1} x_{2}^{2^{s+1}-1} x_{3}^{7.2^{s}-1} \text {, } \\
& b_{s, 28}=x_{2}^{2^{s+1}-1} x_{3}^{7.2^{s}-1} \text {, } \\
& b_{s, 31}=x_{1}^{2^{s+1}-1} x_{3}^{7.2^{s}-1}, \\
& b_{s, 34}=x_{1}^{2^{s+1}-1} x_{2}^{7.2^{s}-1}, \\
& b_{s, 37}=x_{1}^{2^{s+1}-1} x_{2}^{3.2^{s}-1} x_{3}^{5.2^{s}-1}, \\
& b_{s, 40}=x_{1}^{3.2^{s}-1} x_{2}^{5.2^{s}-1} \text {. } \\
& b_{s, 2}=x_{2}^{2^{s+2}-1} x_{3}^{2^{s+1}-1} x_{4}^{2^{s+2}-1} \text {, } \\
& b_{s, 5}=x_{1}^{2^{s+2}-1} x_{3}^{2^{s+1}-1} x_{4}^{2^{s+2}-1} \text {, } \\
& b_{s, 8}=x_{1}^{2^{s+2}-1} x_{2}^{2^{s+1}-1} x_{4}^{2^{s+2}-1}, \\
& b_{s, 11}=x_{1}^{2^{s+2}-1} x_{2}^{2^{s+1}-1} x_{3}^{2^{s+2}-1} \text {, } \\
& b_{s, 14}=x_{1}^{2^{s}-1} x_{2}^{2^{s+3}-1} x_{3}^{2^{s+3}-1} \text {, } \\
& b_{s, 17}=x_{2}^{2^{s+3}-1} x_{3}^{2^{s+3}-1} \text {, } \\
& b_{s, 20}=x_{1}^{2^{s+3}-1} x_{3}^{2^{s+3}-1} \text {, } \\
& b_{s, 23}=x_{1}^{2^{s+3}-1} x_{2}^{2^{s+3}-1} \text {, } \\
& b_{s, 26}=x_{1}^{2^{s+1}-1} x_{2}^{2^{s}-1} x_{3}^{7.2^{s}-1} \text {, } \\
& b_{s, 29}=x_{2}^{2^{s}-1} x_{3}^{7.2^{s}-1} \text {, } \\
& b_{s, 32}=x_{1}^{2^{s}-1} x_{3}^{7.2^{s}-1} \text {, } \\
& b_{s, 35}=x_{1}^{2^{s}-1} x_{2}^{7.2^{s}-1} \text {, } \\
& b_{s, 38}=x_{2}^{3.2^{s}-1} x_{3}^{5.2^{s}-1} \text {, }
\end{aligned}
$$$$
b_{s, 3}=x_{2}^{2^{s+2}-1} x_{3}^{2^{s+2}-1} x_{4}^{2^{s+1}-1},
$$$$
b_{s, 6}=x_{1}^{2^{s+2}-1} x_{3}^{2^{s+2}-1} x_{4}^{2^{s+1}-1} \text {, }
$$$$
b_{s, 9}=x_{1}^{2^{s+2}-1} x_{2}^{2^{s+2}-1} x_{4}^{2^{s+1}-1} \text {, }
$$$$
b_{s, 12}=x_{1}^{2^{s+2}-1} x_{2}^{2^{s+2}-1} x_{3}^{2^{s+1}-1},
$$$$
b_{s, 15}=x_{1}^{2^{s+3}-1} x_{2}^{2^{s}-1} x_{3}^{2^{s}-1} \text {, }
$$$$
b_{s, 18}=x_{2}^{2^{s}-1} x_{3}^{2^{s}-1},
$$$$
b_{s, 21}=x_{1}^{2^{s}-1} x_{3}^{2^{s}-1} \text {, }
$$$$
b_{s, 24}=x_{1}^{2^{s}-1} x_{2}^{2^{s}-1}
$$$$
b_{s, 27}=x_{1}^{2^{s+1}-1} x_{2}^{7.2^{s}-1} x_{3}^{2^{s}-1} \text {, }
$$$$
b_{s, 30}=x_{2}^{7.2^{s}-1} x_{3}^{2^{s}-1} \text {, }
$$$$
b_{s, 33}=x_{1}^{7.2^{s}-1} x_{3}^{2^{s}-1} \text {, }
$$$$
b_{s, 36}=x_{1}^{7.2^{s}-1} x_{2}^{2^{s}-1} \text {, }
$$$$
b_{s, 39}=x_{1}^{3.2^{s}-1} x_{3}^{5.2^{s}-1} \text {, }
$$

Then, we have a direct summand decomposition of $\Sigma_{4}$-modules:

$$
\underline{\boldsymbol{Q}_{n_{s}}^{4}}=\Sigma_{4}\left(b_{s, 1}\right) \bigoplus \Sigma_{4}\left(b_{s, 13}\right) \bigoplus \Sigma_{4}\left(b_{s, 25}\right) \bigoplus \Sigma_{4}\left(b_{s, 37}\right),
$$


wherein

$$
\begin{array}{ll}
\Sigma_{4}\left(b_{s, 1}\right)=\left\langle\left\{\left[b_{s, j}\right]: 1 \leqslant j \leqslant 12\right\}\right\rangle ; & \Sigma_{4}\left(b_{s, 13}\right)=\left\langle\left\{\left[b_{s, j}\right]: 13 \leqslant j \leqslant 24\right\}\right\rangle \\
\Sigma_{4}\left(b_{s, 25}\right)=\left\langle\left\{\left[b_{s, j}\right]: 25 \leqslant j \leqslant 36\right\}\right\rangle ; & \Sigma_{4}\left(b_{s, 37}\right)=\left\langle\left\{\left[b_{s, j}\right]: 37 \leqslant j \leqslant 40\right\}\right\rangle .
\end{array}
$$

Denote the bases of $\Sigma_{4}\left(b_{s, j}\right)$ by the sets $\left[\mathscr{B}\left(b_{s, j}\right)\right]$ for $j \in\{1,13,25,37\}$, wherein $\mathscr{B}\left(b_{s, j}\right)=\left\{b_{s, j}\right\}$. Suppose that $f \in\left(P_{4}\right)_{n_{s}}$ such that $[f] \in \Sigma_{4}\left(b_{s, j}\right)^{\Sigma_{4}}$. Then, $\left.f \equiv \sum_{x \in \mathscr{B}\left(b_{s}, j\right.}\right) \gamma_{x} . x$ in which $\gamma_{x} \in k$. By a direct computation, we can see that the action of $\Sigma_{4}$ on $Q_{n_{s}}^{4}$ induces the one of it on $\left[\mathscr{B}\left(b_{s, j}\right)\right]$

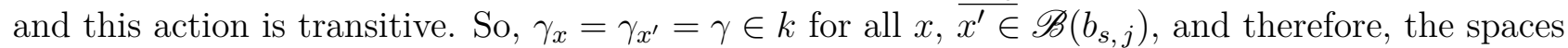
of $\Sigma_{4}$-invariants $\Sigma_{4}\left(b_{s, j}\right)^{\Sigma_{4}}$ are determined as follows.

Lemma 3.2.2. We find that
i) $\Sigma_{4}\left(b_{s, 1}\right)^{\Sigma_{4}}=\left\langle\left[q_{s, 1}\right]\right\rangle$ with $q_{s, 1}:=\sum_{1 \leqslant j \leqslant 12} b_{s, j}$;
ii) $\Sigma_{4}\left(b_{s, 13}\right)^{\Sigma_{4}}=\left\langle\left[q_{s, 2}\right]\right\rangle$ with $q_{s, 2}:=\sum_{13 \leqslant j \leqslant 24} b_{s, j}$;
iii) $\Sigma_{4}\left(b_{s, 25}\right)^{\Sigma_{4}}=\left\langle\left[q_{s, 3}\right]\right\rangle$ with $q_{s, 3}:=\sum_{25 \leqslant j \leqslant 36} b_{s, j}$;
iv) $\Sigma_{4}\left(b_{s, 37}\right)^{\Sigma_{4}}=\left\langle\left[q_{s, 4}\right]\right\rangle$ with $q_{s, 4}:=\sum_{37 \leqslant j \leqslant 40} b_{s, j}$.

By Sum [Su14], the basis of $\overline{\boldsymbol{Q}_{n_{s}}^{4}}$ is a set consisting of all classes represented by the following admissible monomials:

For $s \geqslant 1$,

$$
\begin{aligned}
& b_{s, 41}=x_{1} x_{2}^{2^{s}-1} x_{3}^{2^{s}-1} x_{4}^{2^{s+3}-2}, \\
& b_{s, 42}=x_{1} x_{2}^{2^{s}-1} x_{3}^{2^{s+3}-2} x_{4}^{2^{s}-1}, \\
& b_{s, 43}=x_{1} x_{2}^{2^{s+3}-2} x_{3}^{2^{s}-1} x_{4}^{2^{s}-1} \text {, } \\
& b_{s, 45}=x_{1} x_{2}^{2^{s+1}-2} x_{3}^{2^{s}-1} x_{4}^{7.2^{s}-1} \text {, } \\
& b_{s, 47}=x_{1} x_{2}^{2^{s+1}-2} x_{3}^{3.2^{s}-1} x_{4}^{5.2^{s}-1} \text {, } \\
& b_{s, 49}=x_{1} x_{2}^{2^{s+2}-1} x_{3}^{2^{s+1}-2} x_{4}^{2^{s+2}-1} \text {, } \\
& \begin{array}{l}
b_{s, 51}=x_{1}^{2^{s+2}-1} x_{2} x_{3}^{2^{s+1}-2} x_{4}^{2^{s+2}-1}, \\
b_{s, 53}=x_{1}^{2^{s+2}-1} x_{2}^{2^{s+2}-1} x_{3} x_{4}^{2^{s+1}-2},
\end{array} \\
& b_{s, 55}=x_{1} x_{2}^{2^{s+1}-1} x_{3}^{2^{s+2}-1} x_{4}^{2^{s+2}-2} \text {, } \\
& b_{s, 57}=x_{1} x_{2}^{2^{s+2}-2} x_{3}^{2^{s+2}-1} x_{4}^{2^{s+1}-1} \text {, } \\
& b_{s, 59}=x_{1} x_{2}^{2^{s+2}-1} x_{3}^{2^{s+2}-2} x_{4}^{2^{s+1}-1} \text {, } \\
& b_{s, 61}=x_{1}^{2^{s+1}-1} x_{2} x_{3}^{2^{s+2}-1} x_{4}^{2^{s+2}-2}, \\
& b_{s, 63}=x_{1}^{2^{s+2}-1} x_{2} x_{3}^{2^{s+1}-1} x_{4}^{2^{s+2}-2}, \\
& b_{s, 65}=x_{1}^{2^{s+2}-1} x_{2}^{2^{s+1}-1} x_{3} x_{4}^{2^{s+2}-2}, \\
& b_{s, 67}=x_{1}^{3} x_{2}^{2^{s+2}-3} x_{3}^{2^{s+2}-1} x_{4}^{2^{s+1}-2} \text {, } \\
& b_{s, 69}=x_{1}^{2^{s+2}-1} x_{2}^{3} x_{3}^{2^{s+2}-3} x_{4}^{2^{s+1}-2} \text {, } \\
& b_{s, 71}=x_{1}^{3} x_{2}^{2^{s+2}-3} x_{3}^{2^{s+1}-1} x_{4}^{2^{s+2}-2} \text {, } \\
& b_{s, 44}=x_{1} x_{2}^{2^{s}-1} x_{3}^{2^{s+1}-2} x_{4}^{7.2^{s}-1} \text {, } \\
& b_{s, 46}=x_{1} x_{2}^{2^{s+1}-2} x_{3}^{7.2^{s}-1} x_{4}^{2^{s}-1} \text {, } \\
& b_{s, 48}=x_{1} x_{2}^{2^{s+1}-2} x_{3}^{2^{s+2}-1} x_{4}^{2^{s+2}-1} \text {, } \\
& b_{s, 50}=x_{1} x_{2}^{2^{s+2}-1} x_{3}^{2^{s+2}-1} x_{4}^{2^{s+1}-2}, \\
& b_{s, 52}=x_{1}^{2^{s+2}-1} x_{2} x_{3}^{2^{s+2}-1} x_{4}^{2^{s+1}-2} \text {, } \\
& b_{s, 54}=x_{1} x_{2}^{2^{s+1}-1} x_{3}^{2^{s+2}-2} x_{4}^{2^{s+2}-1}, \\
& b_{s, 56}=x_{1} x_{2}^{2^{s+2}-2} x_{3}^{2^{s+1}-1} x_{4}^{2^{s+2}-1}, \\
& b_{s, 58}=x_{1} x_{2}^{2^{s+2}-1} x_{3}^{2^{s+1}-1} x_{4}^{2^{s+2}-2} \text {, } \\
& b_{s, 60}=x_{1}^{2^{s+1}-1} x_{2} x_{3}^{2^{s+2}-2} x_{4}^{2^{s+2}-1}, \\
& b_{s, 62}=x_{1}^{2^{s+1}-1} x_{2}^{2^{s+2}-1} x_{3} x_{4}^{2^{s+2}-2} \text {, } \\
& b_{s, 64}=x_{1}^{2^{s+2}-1} x_{2} x_{3}^{2^{s+2}-2} x_{4}^{2^{s+1}-1}, \\
& b_{s, 66}=x_{1}^{3} x_{2}^{2^{s+2}-3} x_{3}^{2^{s+1}-2} x_{4}^{2^{s+2}-1}, \\
& b_{s, 68}=x_{1}^{3} x_{2}^{2^{s+2}-1} x_{3}^{2^{s+2}-3} x_{4}^{2^{s+1}-2}, \\
& b_{s, 70}=x_{1}^{3} x_{2}^{2^{s+1}-1} x_{3}^{2^{s+2}-3} x_{4}^{2^{s+2}-2}, \\
& b_{s, 72}=x_{1}^{3} x_{2}^{2^{s+2}-3} x_{3}^{2^{s+2}-2} x_{4}^{2^{s+1}-1} \text {. }
\end{aligned}
$$

For $s=1$,

$$
\begin{array}{lll}
b_{1,73}=x_{1}^{3} x_{2}^{3} x_{3}^{4} x_{4}^{7}, & b_{1,74}=x_{1}^{3} x_{2}^{3} x_{3}^{7} x_{4}^{4}, & b_{1,75}=x_{1}^{3} x_{2}^{7} x_{3}^{3} x_{4}^{4}, \\
b_{1,76}=x_{1}^{7} x_{2}^{3} x_{3}^{3} x_{4}^{4}, & b_{1,77}=x_{1} x_{2} x_{3}^{3} x_{4}^{12}, & b_{1,78}=x_{1} x_{2}^{3} x_{3} x_{4}^{12}, \\
b_{1,79}=x_{1} x_{2}^{3} x_{3}^{12} x_{4}, & b_{1,80}=x_{1}^{3} x_{2} x_{3} x_{4}^{12}, & b_{1,81}=x_{1}^{3} x_{2} x_{3}^{12} x_{4}, \\
b_{1,82}=x_{1} x_{2}^{3} x_{3}^{4} x_{4}^{9}, & b_{1,83}=x_{1}^{3} x_{2} x_{3}^{4} x_{4}^{9}, & b_{1,84}=x_{1} x_{2}^{3} x_{3}^{5} x_{4}^{8}, \\
b_{1,85}=x_{1}^{3} x_{2} x_{3}^{5} x_{4}^{8}, & b_{1,86}=x_{1}^{3} x_{2}^{5} x_{3} x_{4}^{8}, & b_{1,87}=x_{1}^{3} x_{2}^{5} x_{3}^{8} x_{4} .
\end{array}
$$

For $s \geqslant 2$,

$$
\begin{array}{llll}
b_{s, 73}=x_{1}^{2^{s+1}-1} x_{2}^{3} x_{3}^{2^{s+2}-3} x_{4}^{2^{s+2}-2}, & b_{s, 74}=x_{1}^{3} x_{2}^{2^{s+1}-3} x_{3}^{2^{s+2}-2} x_{4}^{2^{s+2}-1} \\
b_{s, 75}=x_{1}^{3} x_{2}^{2^{s+1}-3} x_{3}^{2^{s+2}-1} x_{4}^{2^{s+2}-2}, & b_{s, 76}=x_{1}^{3} x_{2}^{2^{s+2}-1} x_{3}^{2^{s+1}-3} x_{4}^{2^{s+2}-2} \\
b_{s, 77}=x_{1}^{2^{s+2}-1} x_{2}^{3} x_{3}^{2^{s+1}-3} x_{4}^{2^{s+2}-2}, & b_{s, 78}=x_{1}^{7} x_{2}^{2^{s+2}-5} x_{3}^{2^{s+1}-3} x_{4}^{2^{s+2}-2} \\
b_{s, 79}=x_{1}^{7} x_{2}^{2^{s+2}-5} x_{3}^{2^{s+2}-3} x_{4}^{2^{s+1}-2}, & b_{s, 80}=x_{1}^{7} x_{2}^{2^{s+1}-5} x_{3}^{2^{s+2}-3} x_{4}^{2^{s+2}-2} \\
b_{s, 81}=x_{1} x_{2}^{2^{s}-2} x_{3}^{2^{s}-1} x_{4}^{2^{s+3}-1}, & b_{s, 82}=x_{1} x_{2}^{2^{s}-2} x_{3}^{2^{s+3}-1} x_{4}^{2^{s}-1} \\
b_{s, 83}=x_{1} x_{2}^{2^{s}-1} x_{3}^{2^{s}-2} x_{4}^{2^{s+3}-1}, & b_{s, 84}=x_{1} x_{2}^{2^{s}-1} x_{3}^{2^{s+3}-1} x_{4}^{2^{s}-2}
\end{array}
$$




\begin{tabular}{|c|c|}
\hline $\begin{array}{l}b_{s, 85}=x_{1} x_{2}^{2^{s+3}-1} x_{3}^{2^{s}-2} x_{4}^{2^{s}-1}, \\
b_{s, 87}=x_{1}^{2^{s}-1} x_{2} x_{3}^{2^{s}-2} x_{4}^{2^{s+3}-1}, \\
b_{s, 89}=x_{1}^{2^{s}-1} x_{2}^{2^{s+3}-1} x_{3} x_{4}^{2^{s}-2}, \\
b_{s, 91}=x_{1}^{2^{s+3}-1} x_{2} x_{3}^{2^{s}-1} x_{4}^{2^{s}-2}, \\
b_{s, 93}=x_{1}^{2^{s}-1} x_{2} x_{3}^{2^{s}-1} x_{4}^{2^{s+3}-2}, \\
b_{s, 95}=x_{1}^{2^{s}-1} x_{2}^{2^{s}-1} x_{3} x_{4}^{2^{s+3}-2}, \\
b_{s, 97}=x_{1} x_{2}^{2^{s+1}-1} x_{3}^{2^{s}-2} x_{4}^{7.2^{s}-1}, \\
b_{s, 99}=x_{1}^{2^{s+1}-1} x_{2} x_{3}^{2^{s}-2} x_{4}^{7.2^{s}-1}, \\
b_{s, 101}=x_{1}^{2^{s+1}-1} x_{2}^{7.2^{s}-1} x_{3} x_{4}^{2^{s}-2}, \\
b_{s, 103}=x_{1} x_{2}^{2^{s}-1} x_{3}^{2^{s+1}-1} x_{4}^{7.2^{s}-2}, \\
b_{s, 105}=x_{1} x_{2}^{2^{s+1}-1} x_{3}^{7.2^{s}-2} x_{4}^{2^{s}-1}, \\
b_{s, 107}=x_{1}^{2^{s}-1} x_{2}^{2^{s+1}-1} x_{3} x_{4}^{7.2^{s}-2}, \\
b_{s, 109}=x_{1}^{2^{s+1}-1} x_{2} x_{3}^{7.2^{s}-2} x_{4}^{2^{s}-1}, \\
b_{s, 111}=x_{1} x_{2}^{2^{s+1}-1} x_{3}^{3.2^{s}-2} x_{4}^{5.2^{s}-1}, \\
b_{s, 113}=x_{1} x_{2}^{2^{s+1}-1} x_{3}^{3.2^{s}-1} x_{4}^{5.2^{s}-2}, \\
b_{s, 115}=x_{1}^{2^{s+1}-1} x_{2}^{3.2^{s}-1} x_{3} x_{4}^{5.2^{s}-2}, \\
b_{s, 117}=x_{1}^{3} x_{2}^{2^{s+1}-3} x_{3}^{7.2^{s}-1} x_{4}^{2^{s}-2}, \\
b_{s, 119}=x_{1}^{2^{s+1}-1} x_{2}^{3} x_{3}^{7.2^{s}-3} x_{4}^{2^{s}-2}, \\
b_{s, 121}=x_{1}^{3} x_{2}^{2^{s+1}-3} x_{3}^{3.2^{s}-1} x_{4}^{5.2^{s}-2}, \\
b_{s, 123}=x_{1}^{2^{s+1}-1} x_{2}^{3} x_{3}^{3.2^{s}-3} x_{4}^{5.2^{s}-2}, \\
b_{s, 125}=x_{1}^{3} x_{2}^{2^{s+3}-3} x_{3}^{2^{s}-2} x_{4}^{2^{s}-1}, \\
b_{s, 127}=x_{1}^{3} x_{2}^{2^{s}-1} x_{3}^{2^{s+1}-3} x_{4}^{7.2^{s}-2},\end{array}$ & $\begin{array}{l}b_{s, 86}=x_{1} x_{2}^{2^{s+3}-1} x_{3}^{2^{s}-1} x_{4}^{2^{s}-2}, \\
b_{s, 88}=x_{1}^{2^{s}-1} x_{2} x_{3}^{2^{s+3}-1} x_{4}^{2^{s}-2}, \\
b_{s, 90}=x_{1}^{2^{s+3}-1} x_{2} x_{3}^{2^{s}-2} x_{4}^{2^{s}-1}, \\
b_{s, 92}=x_{1}^{2^{s+3}-1} x_{2}^{2^{s}-1} x_{3} x_{4}^{2^{s}-2}, \\
b_{s, 94}=x_{1}^{2^{s}-1} x_{2} x_{3}^{2^{s+3}-2} x_{4}^{2^{s}-1} \\
b_{s, 96}=x_{1} x_{2}^{2^{s}-2} x_{3}^{2^{s+1}-1} x_{4}^{7.2^{s}-1} \\
b_{s, 98}=x_{1} x_{2}^{2^{s+1}-1} x_{3}^{7.2^{s}-1} x_{4}^{2^{s}-2} \\
b_{s, 100}=x_{1}^{2^{s+1}-1} x_{2} x_{3}^{7.2^{s}-1} x_{4}^{2^{s}-} \\
b_{s, 102}=x_{1}^{2^{s}-1} x_{2} x_{3}^{2^{s+1}-2} x_{4}^{7.2^{s}-} \\
b_{s, 104}=x_{1} x_{2}^{2^{s+1}-1} x_{3}^{2^{s}-1} x_{4}^{7.2^{s}-} \\
b_{s, 106}=x_{1}^{2^{s}-1} x_{2} x_{3}^{2^{s+1}-1} x_{4}^{7.2^{s}-} \\
b_{s, 108}=x_{1}^{2^{s+1}-1} x_{2} x_{3}^{2^{s}-1} x_{4}^{7.2^{s}-} \\
b_{s, 110}=x_{1}^{2^{s+1}-1} x_{2}^{2^{s}-1} x_{3} x_{4}^{7.2^{s}-} \\
b_{s, 112}=x_{1}^{2^{s+1}-1} x_{2} x_{3}^{3.2^{s}-2} x_{4}^{5.2^{s}} \\
b_{s, 114}=x_{1}^{2^{s+1}-1} x_{2} x_{3}^{3.2^{s}-1} x_{4}^{5.2^{s}} \\
b_{s, 116}=x_{1}^{3} x_{2}^{2^{s+1}-3} x_{3}^{2^{s}-2} x_{4}^{7.2^{s}-} \\
b_{s, 118}=x_{1}^{3} x_{2}^{2^{s+1}-1} x_{3}^{7.2^{s}-3} x_{4}^{2^{s}-} \\
b_{s, 120}=x_{1}^{3} x_{2}^{2^{s+1}-3} x_{3}^{3.2^{s}-2} x_{4}^{5.2^{s}} \\
b_{s, 122}=x_{1}^{3} x_{2}^{2^{s+1}-1} x_{3}^{3.2^{s}-3} x_{4}^{5.2^{s}} \\
b_{s, 124}=x_{1}^{3} x_{2}^{2^{s}-1} x_{3}^{2^{s+3}-3} x_{4}^{2^{s}-2} \\
b_{s, 126}=x_{1}^{3} x_{2}^{2^{s+3}-3} x_{3}^{2^{s}-1} x_{4}^{2^{s}-2} \\
b_{s, 128}=x_{1}^{3} x_{2}^{2^{s+1}-3} x_{3}^{2^{s}-1} x_{4}^{7.2^{s}-}\end{array}$ \\
\hline
\end{tabular}

For $s=2$,

$b_{2,129}=x_{1}^{3} x_{2}^{3} x_{3}^{3} x_{4}^{28}, \quad b_{2,130}=x_{1}^{3} x_{2}^{3} x_{3}^{28} x_{4}^{3}, \quad b_{2,131}=x_{1}^{3} x_{2}^{3} x_{3}^{4} x_{4}^{27}$, $b_{2,132}=x_{1}^{3} x_{2}^{3} x_{3}^{7} x_{4}^{24}, \quad b_{2,133}=x_{1}^{3} x_{2}^{7} x_{3}^{3} x_{4}^{24}, \quad b_{2,134}=x_{1}^{7} x_{2}^{3} x_{3}^{3} x_{4}^{24}$ $b_{2,135}=x_{1}^{7} x_{2}^{7} x_{3}^{9} x_{4}^{14}$.

For $s \geqslant 3$,

$$
\begin{array}{lll}
b_{s, 129}=x_{1}^{3} x_{2}^{2^{s+1}-3} x_{3}^{7.2^{s}-2} x_{4}^{2^{s}-1}, & b_{s, 130}=x_{1}^{2^{s}-1} x_{2}^{3} x_{3}^{2^{s+1}-3} x_{4}^{7.2^{s}-2}, \\
b_{s, 131}=x_{1}^{2^{s}-1} x_{2}^{3} x_{3}^{2^{s+3}-3} x_{4}^{2^{s}-2}, & b_{s, 132}=x_{1}^{3} x_{2}^{2^{s}-3} x_{3}^{2^{s}-2} x_{4}^{2^{s+3}-1}, \\
b_{s, 133}=x_{1}^{3} x_{2}^{2^{s}-3} x_{3}^{2^{s+3}-1} x_{4}^{2^{s}-2}, & b_{s, 134}=x_{1}^{3} x_{2}^{2^{s+3}-1} x_{3}^{2^{s}-3} x_{4}^{2^{s}-2}, \\
b_{s, 135}=x_{1}^{2^{s+3}-1} x_{2}^{3} x_{3}^{2^{s}-3} x_{4}^{2^{s}-2}, & & b_{s, 136}=x_{1}^{3} x_{2}^{2^{s}-3} x_{3}^{2^{s}-1} x_{4}^{2^{s+3}-2}, \\
b_{s, 137}=x_{1}^{3} x_{2}^{2^{s}-3} x_{3}^{2^{s+3}-2} x_{4}^{2^{s}-1}, & & b_{s, 138}=x_{1}^{3} x_{2}^{2^{s}-1} x_{3}^{2^{s}-3} x_{4}^{2^{s+3}-2}, \\
b_{s, 139}=x_{1}^{2^{s}-1} x_{2}^{3} x_{3}^{2^{s}-3} x_{4}^{2^{s+3}-2}, & b_{s, 140}=x_{1}^{3} x_{2}^{2^{s}-3} x_{3}^{2^{s+1}-2} x_{4}^{7.2^{s}-1}, \\
b_{s, 141}=x_{1}^{3} x_{2}^{2^{s}-3} x_{3}^{2^{s+1}-1} x_{4}^{7.2^{s}-2}, & b_{s, 142}=x_{1}^{3} x_{2}^{2^{s+1}-1} x_{3}^{2^{s}-3} x_{4}^{7.2^{s}-2}, \\
b_{s, 143}=x_{1}^{2^{s+1}-1} x_{2}^{3} x_{3}^{2^{s}-3} x_{4}^{7.2^{s}-2}, & b_{s, 144}=x_{1}^{7} x_{2}^{2^{s+3}-5} x_{3}^{2^{s}-3} x_{4}^{2^{s}-2}, \\
b_{s, 145}=x_{1}^{7} x_{2}^{2^{s+1}-5} x_{3}^{2^{s}-3} x_{4}^{7.2^{s}-2}, & b_{s, 146}=x_{1}^{7} x_{2}^{2^{s+1}-5} x_{3}^{3.2^{s}-3} x_{4}^{5.2^{s}-2}, \\
b_{s, 147}=x_{1}^{7} x_{2}^{2^{s+1}-5} x_{3}^{7.2^{s}-3} x_{4}^{2^{s}-2} . & &
\end{array}
$$

For $s=3$,

$$
b_{3,148}=x_{1}^{7} x_{2}^{7} x_{3}^{7} x_{4}^{56}, \quad b_{3,149}=x_{1}^{7} x_{2}^{7} x_{3}^{9} x_{4}^{54}, \quad b_{3,150}=x_{1}^{7} x_{2}^{7} x_{3}^{57} x_{4}^{6} .
$$

For $s \geqslant 4$,

$$
b_{s, 148}=x_{1}^{7} x_{2}^{2^{s}-5} x_{3}^{2^{s}-3} x_{4}^{2^{s+3}-2}, \quad b_{s, 149}=x_{1}^{7} x_{2}^{2^{s}-5} x_{3}^{2^{s+1}-3} x_{4}^{7.2^{s}-2}, \quad b_{s, 150}=x_{1}^{7} x_{2}^{2^{s}-5} x_{3}^{2^{s+3}-3} x_{4}^{2^{s}-2} .
$$

Remark 3.2.3. Based upon the bases of the spaces $\boldsymbol{Q}_{n_{s}}^{4}$ above, $\omega\left(b_{s, j}\right)$ is one of the following sequences:

$$
\omega_{(s, 1)}:=\underbrace{(3,3, \ldots, 3}_{(s+1) \text { times of } 3}, 2), \omega_{(s, 2)}:=\underbrace{(3,3, \ldots, 3}_{s \text { times of } 3}, 1,1,1) .
$$

Moreover, since $\omega_{(s, 2)}$ is the weight vector of the minimal spike $b_{s, 15},[x]_{\omega_{(s, 2)}}=[x]$ for all monomials $x$ in $\left(P_{4}\right)_{n_{s}}$. 
Case $s=1$. From the admissible basis of $\overline{\boldsymbol{Q}_{n_{1}}^{4}}$ above, we have a direct summand decomposition of $\Sigma_{4}$-modules: $\overline{Q_{n_{1}}^{4}}=\mathcal{M}_{1} \oplus \mathcal{M}_{2}$, wherein $\mathcal{M}_{1}=\left\langle\left\{\left[b_{1, j}\right]: j=41, \ldots, 47,77, \ldots, 87\right\}\right\rangle$ and $\mathcal{M}_{2}=$ $\left\langle\left\{\left[b_{1, j}\right]: j=48, \ldots, 76\right\}\right\rangle$. The below technical claim is pivotal for our further considerations.

Lemma 3.2.4. The following assertions are true:

i) $\mathcal{M}_{1}^{\Sigma_{4}}=\langle[\widetilde{\zeta}]\rangle$ with $\widetilde{\zeta}=b_{1,41}+b_{1,42}+b_{1,78}+b_{1,79}+b_{1,80}+b_{1,81}+b_{1,86}+b_{1,87}$;

ii) $\mathcal{M}_{2}^{\Sigma_{4}}=0$.

Proof. We prove i) in detail. By a similar technique, we also get ii). Suppose that $f \in\left(P_{4}\right)_{n_{1}}$ such that $[f] \in \mathcal{M}_{1}^{\Sigma_{4}}$. Then, we have

$$
f \equiv\left(\sum_{41 \leqslant j \leqslant 47} \gamma_{j} b_{1, j}+\sum_{77 \leqslant j \leqslant 87} \gamma_{j} b_{1, j}\right) .
$$

Direct calculating $\sigma_{i}(f)$ in terms of $b_{1, j}, j \in\{41, \ldots, 47,77, \ldots, 87\}$ modulo $\left(\widehat{A}\left(P_{4}\right)_{n_{1}}\right)$ and using the relations $\left(\sigma_{i}(f)+f\right) \equiv 0$, for $i \in\{1,2,3\}$, we get

$$
\begin{aligned}
\left(\sigma_{1}(f)+f\right) \equiv & \left(\gamma_{45} b_{1,41}+\gamma_{45} b_{1,46}+\left(\gamma_{45}+\gamma_{47}\right) b_{1,44}+\left(\gamma_{46}+\gamma_{47}\right) b_{1,77}\right. \\
& +\left(\gamma_{43}+\gamma_{78}+\gamma_{80}\right) b_{1,78}+\left(\gamma_{43}+\gamma_{79}+\gamma_{81}\right) b_{1,79} \\
& \left.+\left(\gamma_{82}+\gamma_{83}\right) b_{1,82}+\left(\gamma_{84}+\gamma_{85}\right) b_{1,84}+\gamma_{43} b_{1,86}+\text { others terms }\right) \equiv 0, \\
\left(\sigma_{2}(f)+f\right) \equiv & \left(\gamma_{43} b_{1,41}+\left(\gamma_{42}+\gamma_{43}+\gamma_{87}\right) b_{1,42}+\left(\gamma_{42}+\gamma_{43}+\gamma_{81}\right) b_{1,43}\right. \\
& +\left(\gamma_{44}+\gamma_{45}+\gamma_{83}\right) b_{1,44}+\left(\gamma_{46}+\gamma_{79}+\gamma_{87}\right) b_{1,46}+\left(\gamma_{47}+\gamma_{82}\right) b_{1,47} \\
& +\left(\gamma_{77}+\gamma_{78}+\gamma_{87}\right) b_{1,77}+\left(\gamma_{77}+\gamma_{78}+\gamma_{81}\right) b_{1,78}+\left(\gamma_{46}+\gamma_{79}+\gamma_{81}\right) b_{1,79} \\
& +\gamma_{83} b_{1,80}+\left(\gamma_{81}+\gamma_{87}\right) b_{1,81}+\left(\gamma_{81}+\gamma_{87}\right) b_{1,81}+\left(\gamma_{85}+\gamma_{86}+\gamma_{87}\right) b_{1,85} \\
& \left.+\left(\gamma_{81}+\gamma_{85}+\gamma_{86}\right) b_{1,86}+\text { others terms }\right) \equiv 0, \\
\left(\sigma_{3}(f)+f\right) \equiv & \left(\left(\gamma_{41}+\gamma_{42}\right) b_{1,41}+\left(\gamma_{44}+\gamma_{47}\right) b_{1,44}+\left(\gamma_{45}+\gamma_{46}\right) b_{1,45}+\left(\gamma_{78}+\gamma_{79}\right) b_{1,78}\right. \\
& +\left(\gamma_{80}+\gamma_{81}\right) b_{1,80}+\left(\gamma_{82}+\gamma_{84}\right) b_{1,82}+\left(\gamma_{83}+\gamma_{85}\right) b_{1,83} \\
& \left.+\left(\gamma_{86}+\gamma_{87}\right) b_{1,86}+\text { others terms }\right) \equiv 0 .
\end{aligned}
$$

Thus $\gamma_{41}=\gamma_{42}=\gamma_{78}=\cdots=\gamma_{81}=\gamma_{86}=\gamma_{87}$ and $\gamma_{j}=0$ with $j \notin\{41,42,78,79,80,81,86,87\}$. The lemma follows.

Now , assume that $[h] \in\left(\boldsymbol{Q}_{n_{1}}^{4}\right)^{G(4)}$, then since $\Sigma_{4} \subset G(4)$, by Lemmas 3.2.2 and 3.2.4, we have

$$
h \equiv\left(\beta_{1} q_{2,2}+\beta_{2} q_{2,3}+\beta_{3} q_{2,4}+\beta_{4} \widetilde{\zeta}\right),
$$

wherein $\beta_{i} \in k$ for every $i$. Computing $\sigma_{4}(h)$ in terms of $b_{1, j}, 1 \leqslant i \leqslant 87$ modulo $\left(\widehat{A}\left(P_{4}\right)_{n_{1}}\right)$ and using the relation $\left(\sigma_{4}(f)+f\right) \equiv 0$, we obtain

$$
\left(\sigma_{4}(f)+f\right) \equiv\left(\beta_{1} b_{1,13}+\left(\beta_{1}+\beta_{2}\right) b_{1,20}+\left(\beta_{2}+\beta_{3}\right) b_{1,31}+\text { others terms }\right) \equiv 0 .
$$

This leads to $\beta_{1}=\beta_{2}=\beta_{3}=0$.

Case $s \geqslant 2$. It is sufficient to show that the spaces $\left(\left(\boldsymbol{Q}_{n_{s}}^{4}\right)^{\omega_{(s, 1)}}\right)^{G(4)}$ and $\left(\left(\boldsymbol{Q}_{n_{s}}^{4}\right)^{\omega_{(s, 2)}}\right)^{G(4)}$ are trivial for every $s \geqslant 2$. Indeed, we prove this statement for the invariant $\left(\left(\boldsymbol{Q}_{n_{s}}^{4}\right)^{\omega_{(s, 1)}}\right)^{G(4)}$ with $s \geqslant 4$. The other cases can be proved by similar computations.

We have $\left(\boldsymbol{Q}_{n_{s}}^{4}\right)^{\omega_{(s, 1)}} \cong\left({\underline{\boldsymbol{Q}_{n_{s}}^{4}}}^{4}\right)^{\omega_{(s, 1)}} \bigoplus\left(\overline{\boldsymbol{Q}_{n_{s}}^{4}}\right)^{\omega_{(s, 1)}}$, wherein

$$
\left(\underline{\boldsymbol{Q}_{n_{s}}^{4}}\right)^{\omega_{(s, 1)}}=\left\langle\left\{\left[b_{s, j}\right]_{\omega_{(s, 1)}}: 1 \leqslant j \leqslant 12\right\}\right\rangle \text {, and }\left(\overline{\boldsymbol{Q}_{n_{s}}^{4}}\right)^{\omega_{(s, 1)}}=\left\langle\left\{\left[b_{s, j}\right]_{\omega_{(s, 1)}}: 48 \leqslant j \leqslant 80\right\}\right\rangle .
$$

Then, it is easy to check that

$$
\left(\overline{\boldsymbol{Q}_{n_{s}}^{4}}\right)^{\omega_{(s, 1)}} \cong \Sigma_{4}\left(b_{s, 48}\right) \bigoplus \Sigma_{4}\left(b_{s, 54}, b_{s, 66}\right) \bigoplus \mathcal{M}
$$


wherein

$$
\begin{array}{ll}
\Sigma_{4}\left(b_{s, 48}\right) & =\left\langle\left\{\left[b_{s, j}\right]_{\omega_{(s, 1)}}: j=48, \ldots, 53\right\}\right\rangle, \\
\Sigma_{4}\left(b_{s, 54}, b_{s, 66}\right) & =\left\langle\left\{\left[b_{s, j}\right]_{\omega_{(s, 1)}}: j=54, \ldots, 69,74,75,76,77\right\}\right\rangle, \\
\mathcal{M} & =\left\langle\left\{\left[b_{s, j}\right]_{\omega_{(s, 1)}}: j=70,71,72,73,78,79,80\right\}\right\rangle .
\end{array}
$$

Lemma 3.2.5. The following hold:

i) $\Sigma_{4}\left(b_{s, 48}\right)^{\Sigma_{4}}=\left\langle\left[\widehat{q_{s, 2}}\right]_{\omega_{(s, 1)}}\right\rangle$ with $\widehat{q_{s, 2}}:=\sum_{48 \leqslant j \leqslant 53} b_{s, j}$.

ii) $\Sigma_{4}\left(b_{s, 54}, b_{s, 66}\right)^{\Sigma_{4}}=\left\langle\left[\widehat{q_{s, 3}}\right]_{\omega_{(s, 1)}}\right\rangle$ with $\widehat{q_{s, 3}}:=\sum_{54 \leqslant j \leqslant 69} b_{s, j}+\sum_{74 \leqslant j \leqslant 77} b_{s, j}$.

iii) $\mathcal{M}^{\Sigma_{4}}=\left\langle\left[\widehat{q_{s, 4}}\right]_{\omega_{(s, 1)}},\left[\widehat{q_{s, 5}}\right]_{\omega_{(s, 1)}}\right\rangle$ with

$$
\widehat{q_{s, 4}}:=\sum_{70 \leqslant j \leqslant 73} b_{s, j}+b_{s, 78}+b_{s, 79}, \widehat{q_{s, 5}}:=\sum_{70 \leqslant j \leqslant 73} b_{s, j}+b_{s, 80} .
$$

Proof. We prove Part iii) in detail. The others parts can be computed by a similar idea. Computing the general case is very technical. Indeed, we have the set $\left\{\left[b_{s, j}\right]_{\omega_{(s, 1)}}: j=70,71,72,73,78,79,80\right\}$, which is an admissible basis of $\mathcal{M}$. Suppose that $[f] \in \mathcal{M}^{\Sigma_{4}}$, then

$$
f \equiv_{\omega_{(s, 1)}}\left(\sum_{70 \leqslant j \leqslant 73} \gamma_{j} b_{s, j}+\sum_{78 \leqslant j \leqslant 80} \gamma_{j} b_{s, j}\right), \gamma_{j} \in k \text { for every } j .
$$

Using the homomorphisms $\sigma_{i}: P_{4} \longrightarrow P_{4}, i=1,2,3$, we have the following equalities:

$$
\begin{aligned}
\sigma_{1}(f) \equiv & \omega_{(s, 1)}\left(\gamma_{73} b_{s, 70}+\gamma_{70} b_{s, 73}+\gamma_{71} x_{1}^{2^{s+2}-3} x_{2}^{3} x_{3}^{2^{s+1}-1} x_{4}^{2^{s+2}-2}\right. \\
& +\gamma_{72} x_{1}^{2^{s+2}-3} x_{2}^{3} x_{3}^{2^{s+2}-2} x_{4}^{2^{s+1}-1}+\gamma_{78} x_{1}^{2^{s+2}-5} x_{2}^{7} x_{3}^{2^{s+1}-3} x_{4}^{2^{s+2}-2} \\
& \left.+\gamma_{79} x_{1}^{2^{s+2}-5} x_{2}^{7} x_{3}^{2^{s+2}-3} x_{4}^{2^{s+1}-2}+\gamma_{80} x_{1}^{2^{s+1}-5} x_{2}^{7} x_{3}^{2^{s+2}-3} x_{4}^{2^{s+2}-2}\right), \\
\sigma_{2}(f) \equiv & { }_{\omega_{(s, 1)}}\left(\gamma_{71} b_{s, 70}+\gamma_{70} b_{s, 71}+\gamma_{72} x_{1}^{3} x_{2}^{2^{s+2}-2} x_{3}^{2^{s+2}-3} x_{4}^{2^{s+1}-1}\right. \\
& +\gamma_{73} x_{1}^{2^{s+1}-1} x_{2}^{2^{s+2}-3} x_{3}^{3} x_{4}^{2^{s+2}-2}+\gamma_{78} x_{1}^{7} x_{2}^{2^{s+1}-3} x_{3}^{2^{s+2}-5} x_{4}^{2^{s+2}-2} \\
& \left.+\gamma_{79} x_{1}^{7} x_{2}^{2^{s+2}-3} x_{3}^{2^{s+2}-5} x_{4}^{2^{s+1}-2}+\gamma_{80} x_{1}^{7} x_{2}^{2^{s+2}-3} x_{3}^{2^{s+1}-5} x_{4}^{2^{s+2}-2}\right), \\
\sigma_{3}(f) \equiv & \omega_{(s, 1)}\left(\gamma_{72} b_{s, 71}+\gamma_{71} b_{s}, 72+\gamma_{70} x_{1}^{3} x_{2}^{2^{s+1}-1} x_{3}^{2^{s+2}-2} x_{4}^{2^{s+2}-3}\right. \\
& +\gamma_{73} x_{1}^{2^{s+1}-1} x_{2}^{3} x_{3}^{2^{s+2}-2} x_{4}^{2^{s+2}-3}+\gamma_{78} x_{1}^{7} x_{2}^{2^{s+2}-5} x_{3}^{2^{s+2}-2} x_{4}^{2^{s+1}-3} \\
& \left.+\gamma_{79} x_{1}^{7} x_{2}^{2^{s+2}-5} x_{3}^{2^{s+1}-2} x_{4}^{2^{s+2}-3}+\gamma_{80} x_{1}^{7} x_{2}^{2^{s+1}-5} x_{3}^{2^{s+2}-2} x_{4}^{2^{s+2}-3}\right) .
\end{aligned}
$$

Using the actions of the Steenrod squares and the Cartan formula, we get

$$
\begin{array}{ll}
x_{1}^{3} x_{2}^{2^{s+1}-1} x_{3}^{2^{s+2}-2} x_{4}^{2^{s+2}-3} & \equiv_{\omega_{(s, 1)}} b_{s, 70}, \mid x_{1}^{3} x_{2}^{2^{s+2}-2} x_{3}^{2^{s+2}-3} x_{4}^{2^{s+1}-1} \equiv_{\omega_{(s, 1)}} b_{s, 72}, \\
x_{1}^{7} x_{2}^{2^{s+1}-5} x_{3}^{2^{s+2}-2} x_{4}^{2^{s+2}-3} & \equiv_{\omega_{(s, 1)}} b_{s, 80}, \mid x_{1}^{7} x_{2}^{2^{s+1}-3} x_{3}^{2^{s+2}-5} x_{4}^{2^{s+2}-2} \equiv_{\omega_{(s, 1)}} b_{s, 80}, \\
x_{1}^{7} x_{2}^{2^{s+2}-5} x_{3}^{2^{s+1}-2} x_{4}^{2^{s+2}-3} & \equiv_{\omega_{(s, 1)}} b_{s, 78}, \mid x_{1}^{7} x_{2}^{2^{s+2}-5} x_{3}^{2^{s+2}-2} x_{4}^{2^{s+1}-3} \equiv_{\omega_{(s, 1)}} b_{s, 79}, \\
x_{1}^{7} x_{2}^{2^{s+2}-3} x_{3}^{2^{s+1}-5} x_{4}^{2^{s+2}-2} & \equiv_{\omega_{(s, 1)}} b_{s, 78}, \mid x_{1}^{7} x_{2}^{2^{s+2}-3} x_{3}^{2^{s+2}-5} x_{4}^{2^{s+1}-2} \equiv_{\omega_{(s, 1)}} b_{s, 79}, \\
x_{1}^{2^{s+1}-5} x_{2}^{7} x_{3}^{2^{s+2}-3} x_{4}^{2^{s+2}-2} & \equiv_{\omega_{(s, 1)}} b_{s, 80}, \mid x_{1}^{2^{s+1}-1} x_{2}^{3} x_{3}^{2^{s+2}-2} x_{4}^{2^{s+2}-3} \equiv_{\omega_{(s, 1)}} b_{s, 73}, \\
x_{1}^{2^{s+1}-1} x_{2}^{2^{s+2}-3} x_{3}^{3} x_{4}^{2^{s+2}-2} & \equiv_{\omega_{(s, 1)}}\left(b_{s, 73}+b_{s, 78}+b_{s, 80}\right), \\
x_{1}^{2^{s+2}-5} x_{2}^{7} x_{3}^{2^{s+1}-3} x_{4}^{2^{s+2}-2} & \equiv_{\omega_{(s, 1)}}\left(b_{s, 78}+b_{s, 80}\right), \\
x_{1}^{2^{s+2}-5} x_{2}^{7} x_{3}^{2^{s+2}-3} x_{4}^{2^{s+1}-2} & \equiv_{\omega_{(s, 1)}}\left(b_{s, 79}+b_{s, 80}\right), \\
x_{1}^{2^{s+2}-3} x_{2}^{3} x_{3}^{2^{s+1}-1} x_{4}^{2^{s+2}-2} & \equiv_{\omega_{(s, 1)}}\left(b_{s, 71}+b_{s, 80}\right), \\
x_{1}^{2^{s+2}-3} x_{2}^{3} x_{3}^{2^{s+2}-2} x_{4}^{2^{s+1}-1} & \equiv_{\omega_{(s, 1)}}\left(b_{s, 72}+b_{s, 80}\right) .
\end{array}
$$


Combining the above computations and the relations $\sigma_{i}(f) \equiv_{\omega_{(s, 1)}} f$ for $1 \leqslant i \leqslant 3$, we obtain $\gamma_{78}=\gamma_{79}$ and $\gamma_{j}+\gamma_{79}+\gamma_{80}=0$, for $70 \leqslant j \leqslant 73$. The lemma is proved.

Now, let $\left.[g] \in\left(\boldsymbol{Q}_{n_{s}}^{4}\right)^{\omega_{(s, 1)}}\right)^{G(4)}$, by Lemmas 3.2.2 and 3.2.5, we have

$$
g \equiv_{\omega_{(s, 1)}}\left(\rho_{1} q_{s, 1}+\rho_{2} \widehat{q_{s, 2}}+\rho_{3} \widehat{q_{s, 3}}+\rho_{4} \widehat{q_{s, 4}}+\rho_{5} \widehat{q_{s, 5}}\right)
$$

with $\rho_{i} \in k$ for all $i, 1 \leqslant i \leqslant 5$. Direct computing from the relation $\left(\sigma_{4}(g)+g\right) \equiv_{\omega_{(s, 1)}} 0$, we get

$$
\begin{gathered}
\left(\sigma_{4}(g)+g\right) \equiv_{\omega_{(s, 1)}}\left(\left(\rho_{1}+\rho_{2}\right) b_{s, 1}+\left(\rho_{1}+\rho_{3}\right) b_{s, 2}+\rho_{1} b_{s, 7}+\left(\rho_{4}+\rho_{5}\right) b_{s, 58}\right. \\
\left.+\rho_{4} b_{s, 76}+\text { others terms }\right) \equiv_{\omega_{(s, 1)}} 0
\end{gathered}
$$

and therefore $\rho_{i}=0$ for all $i$. This completes the proof of the proposition.

Now, combining Proposition 3.2.1 with the fact that $\langle\zeta, \widetilde{\zeta}\rangle=1$, we obtain

$$
k \otimes_{G(4)} P_{A}\left(\left(P_{4}\right)_{n_{s}}^{*}\right)=\left(\left(Q_{n_{s}}^{4}\right)^{G(4)}\right)^{*}= \begin{cases}\left\langle([\widetilde{\zeta}])^{*}\right\rangle=\langle[\zeta]\rangle & \text { if } s=1, \\ 0 & \text { otherwise. }\end{cases}
$$

The theorem is proved.

\subsection{Proof of Theorem 2.1.8}

We put $n_{s, t}:=2^{s+t+1}+2^{s+1}-3$, then from a result in [Su14], $\boldsymbol{Q}_{n_{s, t}}^{4} \cong\left(\boldsymbol{Q}_{n_{s, t}}^{4}\right)^{\omega_{(s, t, 1)}} \bigoplus\left(\boldsymbol{Q}_{n_{s, t}}^{4}\right)^{\omega_{(s, t, 2)}}$, wherein

$$
\omega_{(s, t, 1)}:=\underbrace{(3,3, \ldots, 3}_{s \text { times }}, \underbrace{2,2, \ldots, 2)}_{(t+1) \text { times }} \text {, and } \omega_{(s, t, 2)}:=\underbrace{(3,3, \ldots, 3}_{(s+1) \text { times }}, \underbrace{2,2, \ldots, 2)}_{(t-1) \text { times }} .
$$

By using an admissible basis for $\left(\boldsymbol{Q}_{n_{s, t}}^{4}\right)^{\omega_{(s, t, j)}}$ (see [Su14]), we obtain

Proposition 3.3.1. Let $s$ and $t$ be positive integers such that $t \geqslant 4$. Then,

$$
\operatorname{dim}\left(\left(Q_{n_{s, t}}^{4}\right)^{\omega_{(s, t, j)}}\right)^{G(4)}= \begin{cases}0 & \text { if } j=1 \text { and } s \in\{1,2\} \\ 1 & \text { if } j=2 \text { and } s \in\{1,2\} \\ 1 & \text { if } j \in\{1,2\} \text { and } s \geqslant 3\end{cases}
$$

Since the proof of the proposition is similar to that of Proposition 3.1.7, we omit details here. On the other hand, as $\zeta_{s, t, 1}=a_{2}^{\left(2^{s+1}-1\right)} a_{3}^{\left(2^{s+t}-1\right)} a_{4}^{\left(2^{s+t}-1\right)}$ and $\zeta_{s, t, 2}=a_{2}^{\left(2^{s}-1\right)} a_{3}^{\left(2^{s}-1\right)} a_{4}^{\left(2^{s+t+1}-1\right)}$ belong to $\operatorname{Ext}_{A}^{0, n_{s, t}}\left(P_{4}, k\right)$, by Theorem 1.2, $\zeta_{s, t, 1}$ and $\zeta_{s, t, 2}$ are representative of the non-zero elements $h_{0} h_{s+1} h_{s+t}^{2}$ and $h_{0} h_{s}^{2} h_{s+t+1} \in \operatorname{Ext}_{A}^{4, n_{s, t}+4}(k, k)$ respectively. Moreover, by Theorem 2.1, the $k$-vector space $\operatorname{Ext}_{A}^{4, n_{s, t}+4}(k, k)$ has dimension 2 for all $s>0$ and $t>3$. In addition, $k \otimes_{G(4)} P_{A}\left(\left(P_{4}\right)_{n_{s, t}}^{*}\right)=$ $\left\langle\left[\zeta_{s, t, 1}\right]\right\rangle$ for $s=1,2$ and $k \otimes_{G(4)} P_{A}\left(\left(P_{4}\right)_{n_{s, t}}^{*}\right)=\left\langle\left[\zeta_{s, t, 1}\right],\left[\zeta_{s, t, 2}\right]\right\rangle$ for all $s \geqslant 3$. This straightforwardly follows from Proposition 3.3.1, which we leave to be proved by the interested reader. The theorem follows.

\subsection{Proof of Theorem 2.2.1}

For simplicity, we prove the theorem for $s \geqslant 4$. The others cases use a similar technique. We denote by $n_{s}=2^{s+1}+2^{s}-2$. Based upon a result in Sum [Su14], we have that

$$
\operatorname{Ker}\left[\overline{S q}^{0}\right]_{n_{s}}=\left(\boldsymbol{Q}_{n_{s}}^{4}\right)^{\omega_{(s)}}=\left(\underline{\boldsymbol{Q}_{n_{s}}^{4}}\right)^{\omega_{(s)}} \bigoplus\left(\overline{\boldsymbol{Q}_{n_{s}}^{4}}\right)^{\omega_{(s)}}, \quad \omega_{(s)}:=\underbrace{(2,2, \ldots, 2}_{s \text { times of } 2}, 1) .
$$

and that the admissible monomial basis of $\left(\underline{\boldsymbol{Q}_{n_{s}}^{4}}\right)^{\omega_{(s)}}$ is a set consisting of all classes represented by the following monomials:

For $s=1$, 

$c_{1,1}=x_{3} x_{4}^{3}$,
$c_{1,2}=x_{3}^{3} x_{4}$,
$c_{1,3}=x_{2} x_{4}^{3}$,
$c_{1,4}=x_{2} x_{3}^{3}$,
$c_{1,5}=x_{2}^{3} x_{4}$,
$c_{1,6}=x_{2}^{3} x_{3}$,
$c_{1,7}=x_{1} x_{4}^{3}$,
$c_{1,8}=x_{1} x_{3}^{3}$,
$c_{1,9}=x_{1} x_{2}^{3}$,
$c_{1,10}=x_{1}^{3} x_{4}$,
$c_{1,11}=x_{1}^{3} x_{3}$,
$c_{1,12}=x_{1}^{3} x_{2}$,
$c_{1,13}=x_{2} x_{3} x_{4}^{2}$,
$c_{1,14}=x_{2} x_{3}^{2} x_{4}$,
$c_{1,15}=x_{1} x_{3} x_{4}^{2}$,
$c_{1,16}=x_{1} x_{3}^{2} x_{4}$,
$c_{1,17}=x_{1} x_{2} x_{4}^{2}$,
$c_{1,18}=x_{1} x_{2} x_{3}^{2}$
$c_{1,19}=x_{1} x_{2}^{2} x_{4}$,
$c_{1,20}=x_{1} x_{2}^{2} x_{3}$.

For $s \geqslant 2$,
$c_{s, 1}=x_{2} x_{3}^{2^{s}-2} x_{4}^{2^{s+1}-1}$,
$c_{s, 4}=x_{1} x_{3}^{2^{s}-2} x_{4}^{2^{s+1}-1}$,
$c_{s, 2}=x_{2} x_{3}^{2^{s+1}-1} x_{4}^{2^{s}-2}$,
$c_{s, 3}=x_{2}^{2^{s+1}-1} x_{3} x_{4}^{2^{s}-2}$,
$c_{s, 4}=x_{1} x_{3}^{2^{s}-2} x_{4}^{2^{s+1}-1}$,
$c_{s, 7}=x_{1} x_{2}^{2^{s}-2} x_{4}^{2^{s+1}-1}$,
$c_{s, 5}=x_{1} x_{3}^{2^{s+1}-1} x_{4}^{2^{s}-2}$,
$c_{s, 6}=x_{1}^{2^{s+1}-1} x_{3} x_{4}^{2^{s}-2}$,
$c_{s, 10}=x_{1} x_{2}^{2^{s}-2} x_{3}^{2^{s+1}-1}$,
$c_{s, 8}=x_{1} x_{2}^{2^{s+1}-1} x_{4}^{2^{s}-2}$,
$c_{s, 9}=x_{1}^{2^{s+1}-1} x_{2} x_{4}^{2^{s}-2}$,
$c_{s, 13}=x_{3}^{2^{s}-1} x_{4}^{2^{s+1}-1}$,
$c_{s, 11}=x_{1} x_{2}^{2^{s+1}-1} x_{3}^{2^{s}-2}$,
$c_{s, 12}=x_{1}^{2^{s+1}-1} x_{2} x_{3}^{2^{s}-2}$,
$c_{s, 16}=x_{2}^{2^{s}-1} x_{3}^{2^{s+1}-1}$,
$c_{s, 14}=x_{3}^{2^{s+1}-1} x_{4}^{2^{s}-1}$,
$c_{s, 17}=x_{2}^{2^{s+1}-1} x_{4}^{2^{s}-1}$,
$c_{s, 20}=x_{1}^{2^{s}-1} x_{3}^{2^{s+1}-1}$,
$c_{s, 19}=x_{1}^{2^{s}-1} x_{4}^{2^{s+1}-1}$,
$c_{s, 22}=x_{1}^{2^{s+1}-1} x_{3}^{2^{s}-1}$,
$c_{s, 23}=x_{1}^{2^{s}-1} x_{2}^{2^{s+1}-1}$,
$c_{s, 28}=x_{1} x_{3}^{2^{s}-1} x_{4}^{2^{s+1}-2}$,
$c_{s, 31}=x_{1} x_{2}^{2^{s}-1} x_{4}^{2^{s+1}-2}$,
$c_{s, 29}=x_{1} x_{3}^{2^{s+1}-2} x_{4}^{2^{s}-1}$,
$c_{s, 32}=x_{1} x_{2}^{2^{s+1}-2} x_{4}^{2^{s}-1}$,
$c_{s, 34}=x_{1} x_{2}^{2^{s}-1} x_{3}^{2^{s+1}-2}$,
$c_{s, 35}=x_{1} x_{2}^{2^{s+1}-2} x_{3}^{2^{s}-1}$,
$c_{s, 37}=x_{2}^{3} x_{3}^{2^{s+1}-3} x_{4}^{2^{s}-2}$,
$c_{s, 38}=x_{1}^{3} x_{3}^{2^{s+1}-3} x_{4}^{2^{s}-2}$,
$c_{s, 15}=x_{2}^{2^{s}-1} x_{4}^{2^{s+1}-1}$,
$c_{s, 18}=x_{2}^{2^{s+1}-1} x_{3}^{2^{s}-1}$,
$c_{s, 21}=x_{1}^{2^{s+1}-1} x_{4}^{2^{s}-1}$,
$c_{s, 24}=x_{1}^{2^{s+1}-1} x_{2}^{2^{s}-1}$,
$c_{s, 30}=x_{1}^{2^{s}-1} x_{3} x_{4}^{2^{s+1}-2}$,
$c_{s, 33}=x_{1}^{2^{s}-1} x_{2} x_{4}^{2^{s+1}-2}$,
$c_{s, 36}=x_{1}^{2^{s}-1} x_{2} x_{3}^{2^{s+1}-2}$
$c_{s, 40}=x_{1}^{3} x_{2}^{2^{s+1}-3} x_{3}^{2^{s}-2}$.
$c_{s, 39}=x_{1}^{3} x_{2}^{2^{s+1}-3} x_{4}^{2^{s}-2}$

For $s=2$,

$$
c_{2,41}=x_{2}^{3} x_{3}^{3} x_{4}^{4}, \quad c_{2,42}=x_{1}^{3} x_{3}^{3} x_{4}^{4}, \quad c_{2,43}=x_{1}^{3} x_{2}^{3} x_{4}^{4}, \quad c_{2,44}=x_{1}^{3} x_{2}^{3} x_{3}^{4} .
$$

For $s \geqslant 3$,

$$
\begin{aligned}
& c_{s, 41}=x_{2}^{3} x_{3}^{2^{s}-3} x_{4}^{2^{s+1}-2}, \quad c_{s, 42}=x_{1}^{3} x_{3}^{2^{s}-3} x_{4}^{2^{s+1}-2}, \quad c_{s, 43}=x_{1}^{3} x_{2}^{2^{s}-3} x_{4}^{2^{s+1}-2}, \\
& c_{s, 44}=x_{1}^{3} x_{2}^{2^{s}-3} x_{3}^{2^{s+1}-2} .
\end{aligned}
$$

Lemma 3.4.1. The following statements are true:

i) For $s=1$, we have a direct summand decomposition of $\Sigma_{4}$-modules:

$$
\left(\underline{\boldsymbol{Q}_{n_{1}}^{4}}\right)^{\omega_{(1)}}=\Sigma_{4}\left(c_{1,1}\right) \bigoplus \Sigma_{4}\left(c_{1,13}\right)
$$

wherein $\Sigma_{4}\left(c_{1,1}\right)=\left\langle\left\{\left[c_{1, j}\right]: 1 \leqslant j \leqslant 12\right\}\right\rangle$ and $\Sigma_{4}\left(c_{1,13}\right)=\left\langle\left\{\left[c_{1, j}\right]: 13 \leqslant j \leqslant 20\right\}\right\rangle$. Moreover,

$$
\Sigma_{4}\left(c_{1,1}\right)^{\Sigma_{4}}=\left\langle\left[\sum_{1 \leqslant j \leqslant 12} c_{1, j}\right]\right\rangle, \quad \Sigma_{4}\left(c_{1,13}\right)^{\Sigma_{4}}=0 .
$$

ii) For $s \geqslant 4$, we have a direct summand decomposition of $\Sigma_{4}$-modules:

$$
\left(\underline{\boldsymbol{Q}_{n_{s}}^{4}}\right)^{\omega_{(s)}}=\Sigma_{4}\left(c_{s, 1}\right) \bigoplus \Sigma_{4}\left(c_{s, 13}\right) \bigoplus \Sigma_{4}\left(c_{s, 25}\right) \bigoplus \Sigma_{4}\left(c_{s, 37}\right),
$$

wherein

$$
\begin{aligned}
& \Sigma_{4}\left(c_{s, 1}\right) \quad=\left\langle\left\{\left[c_{s, j}\right]: 1 \leqslant j \leqslant 12\right\}\right\rangle, \quad \Sigma_{4}\left(c_{s, 13}\right)=\left\langle\left\{\left[c_{s, j}\right]: 13 \leqslant j \leqslant 24\right\}\right\rangle, \\
& \Sigma_{4}\left(c_{s, 25}\right)=\left\langle\left\{\left[c_{s, j}\right]: 25 \leqslant j \leqslant 36\right\}\right\rangle, \quad \Sigma_{4}\left(c_{s, 37}\right)=\left\langle\left\{\left[c_{s, j}\right]: 37 \leqslant j \leqslant 44\right\}\right\rangle .
\end{aligned}
$$

iii) $\Sigma_{4}\left(c_{s, j}\right)^{\Sigma_{4}}=0$ for $j=25,37$.

iv) $\Sigma_{4}\left(c_{s, 1}\right)^{\Sigma_{4}}=\left\langle\left[q_{s, 1}\right]\right\rangle$ with $q_{s, 1}:=\sum_{1 \leqslant j \leqslant 12}$.

v) $\Sigma_{4}\left(c_{s, 13}\right)^{\Sigma_{4}}=\left\langle\left[q_{s, 2}\right]\right\rangle$ with $q_{s, 2}:=\sum_{13 \leqslant j \leqslant 24}$.

It is rather straightforward to prove this lemma directly, so we omit the details by leaving them to the interested reader for a direct check. Now, according to [Su14], the admissible bases of $\left(\overline{\boldsymbol{Q}_{n_{s}}^{4}}\right)^{\omega_{(s)}}$ are the sets consisting of all classes represented by the following monomials: 
For $s \geqslant 2$,

$$
\begin{array}{lll}
c_{s, 45}=x_{1} x_{2}^{2^{s}-2} x_{3} x_{4}^{2^{s+1}-2}, & c_{s, 46}=x_{1} x_{2}^{2^{s+1}-2} x_{3} x_{4}^{2^{s}-2}, & c_{s, 47}=x_{1} x_{2}^{2} x_{3}^{2^{s}-3} x_{4}^{2^{s+1}-2}, \\
c_{s, 48}=x_{1} x_{2}^{3} x_{3}^{2^{s}-4} x_{4}^{2^{s+1}-2}, & c_{s, 49}=x_{1} x_{2}^{3} x_{3}^{2^{s+1}-2} x_{4}^{2^{s}-4}, & c_{s, 50}=x_{1}^{3} x_{2} x_{3}^{2^{s}-4} x_{4}^{2^{s+1}-2}, \\
c_{s, 51}=x_{1}^{3} x_{2} x_{3}^{2^{s+1}-2} x_{4}^{2^{s}-4}, & c_{s, 52}=x_{1}^{3} x_{2}^{2^{s+1}-3} x_{3}^{2} x_{4}^{2^{s}-4}, & c_{s, 53}=x_{1} x_{2} x_{3}^{2^{s}-2} x_{4}^{2^{s+1}-2}, \\
c_{s, 54}=x_{1} x_{2} x_{3}^{2^{s+1}-2} x_{4}^{2^{s}-2}, & c_{s, 55}=x_{1} x_{2}^{2} x_{3}^{2^{s+1}-3} x_{4}^{2^{s}-2} .
\end{array}
$$

For $s=2, c_{2,56}=x_{1}^{3} x_{2}^{4} x_{3} x_{4}^{2}$.

For $s \geqslant 3$,

$$
\begin{array}{lll}
c_{s, 56}=x_{1}^{3} x_{2}^{5} x_{3}^{2^{s+1}-6} x_{4}^{2^{s}-4}, & c_{s, 57}=x_{1}^{3} x_{2}^{5} x_{3}^{2^{s}-6} x_{4}^{2^{s+1}-4}, & c_{s, 58}=x_{1} x_{2}^{3} x_{3}^{2^{s+1}-4} x_{4}^{2^{s}-2}, \\
c_{s, 59}=x_{1}^{3} x_{2} x_{3}^{2^{s+1}-4} x_{4}^{2^{s}-2}, & c_{s, 60}=x_{1} x_{2}^{3} x_{3}^{2^{s}-2} x_{4}^{2^{s+1}-4}, & c_{s, 61}=x_{1}^{3} x_{2} x_{3}^{2^{s}-2} x_{4}^{2^{s+1}-4}, \\
c_{s, 62}=x_{1}^{3} x_{2}^{2^{s}-3} x_{3}^{2} x_{4}^{2^{s+1}-4}, & c_{s, 63}=x_{1} x_{2}^{2} x_{3}^{2^{s}-4} x_{4}^{2^{s+1}-1}, & c_{s, 64}=x_{1} x_{2}^{2} x_{3}^{2^{s+1}-1} x_{4}^{2^{s}-4}, \\
c_{s, 65}=x_{1} x_{2}^{2^{s+1}-1} x_{3}^{2} x_{4}^{2^{s}-4}, & c_{s, 66}=x_{1}^{2^{s+1}-1} x_{2} x_{3}^{2} x_{4}^{2^{s}-4}, & c_{s, 67}=x_{1} x_{2}^{2} x_{3}^{2^{s}-1} x_{4}^{2^{s+1}-4}, \\
c_{s, 68}=x_{1} x_{2}^{2} x_{3}^{2^{s+1}-4} x_{4}^{2^{s}-1}, & c_{s, 69}=x_{1} x_{2}^{2^{s}-1} x_{3}^{2} x_{4}^{2^{s+1}-4} .
\end{array}
$$

For $s=3, c_{3,70}=x_{1}^{3} x_{2}^{5} x_{3}^{6} x_{4}^{8}$.

For $s \geqslant 4, c_{s, 70}=x_{1}^{2^{s}-1} x_{2} x_{3}^{2} x_{4}^{2^{s+1}-4}$.

Lemma 3.4.2. The space of $\Sigma_{4}$-invariants $\left(\left(\overline{\boldsymbol{Q}_{n_{s}}^{4}}\right)^{\omega_{(s)}}\right)^{\Sigma_{4}}$ is generated by the classes $\left[q_{s, 3}\right]$ and $\left[q_{s, 4}\right]$, wherein

$$
q_{s, 3}=c_{s, 47}+c_{s, 48}+\sum_{62 \leqslant j \leqslant 67} c_{s, j}, \quad q_{s, 4}=\sum_{58 \leqslant j \leqslant 61} c_{s, j} .
$$

Proof. It should be noticed that $\operatorname{dim}\left(\overline{\boldsymbol{Q}_{n_{s}}^{4}}\right)^{\omega_{(s)}}=26$ with the basis $\left\{\left[c_{s, j}\right]: 45 \leqslant j \leqslant 70\right\}$. Suppose $[g] \in\left(\left(\overline{\boldsymbol{Q}_{n_{s}}^{4}}\right)^{\omega_{(s)}}\right)^{\Sigma_{4}}$, then we have $g \equiv\left(\sum_{45 \leqslant j \leqslant 70} \beta_{j} c_{s, j}\right)$ with $\beta_{j} \in k$. Using Cartan's formula and the relations $\left(\sigma_{i}(g)+g\right) \equiv 0$, for $1 \leqslant i \leqslant 3$, we get:

$$
\begin{aligned}
\sigma_{1}(g)+g \equiv & \left(\left(\beta_{47}+\beta_{48}+\beta_{62}+\beta_{67}+\beta_{68}+\beta_{70}\right) c_{s, 45}+\left(\beta_{48}+\beta_{49}+\beta_{67}+\beta_{69}\right) c_{s, 46}\right. \\
& +\left(\beta_{52}+\beta_{53}\right)\left(c_{s, 52}+c_{s, 53}\right)+\left(\beta_{54}+\beta_{55}\right)\left(c_{s, 54}+c_{s, 55}\right) \\
& +\left(\beta_{48}+\beta_{56}+\beta_{57}+\beta_{67}\right)\left(c_{s, 56}+c_{s, 57}\right)+\left(\beta_{60}+\beta_{61}\right)\left(c_{s, 60}+c_{s, 61}\right) \\
& +\left(\beta_{63}+\beta_{65}\right)\left(c_{s, 63}+c_{s, 65}\right)+\left(\beta_{64}+\beta_{66}\right)\left(c_{s, 64}+c_{s, 66}\right)+\left(\beta_{48}\right. \\
& \left.\left.+\beta_{67}\right)\left(c_{s, 68}+c_{s, 70}\right)\right) \equiv \\
\sigma_{2}(g)+g \equiv & \left(\left(\beta_{45}+\beta_{47}+\beta_{49}+\beta_{54}+\beta_{55}+\beta_{56}+\beta_{65}\right) c_{s, 45}+\left(\beta_{46}+\beta_{48}+\beta_{55}+\beta_{64}+\beta_{69}\right) c_{s, 46}\right. \\
& +\left(\beta_{45}+\beta_{47}+\beta_{56}+\beta_{65}\right) c_{s, 47}+\left(\beta_{46}+\beta_{48}+\beta_{55}+\beta_{64}\right) c_{s, 48}+\left(\beta_{49}+\beta_{54}\right) c_{s, 49} \\
& +\left(\beta_{50}+\beta_{52}\right)\left(c_{s, 50}+c_{s, 52}\right)+\left(\beta_{49}+\beta_{54}+\beta_{69}\right) c_{s, 47}+\beta_{69} c_{s, 55} \\
& +\left(\beta_{49}+\beta_{54}+\beta_{55}+\beta_{69}\right) c_{s, 56}+\left(\beta_{57}+\beta_{68}\right) c_{s, 57}+\left(\beta_{59}+\beta_{60}\right)\left(c_{s, 59}+c_{s, 60}\right) \\
& +\left(\beta_{57}+\beta_{68}\right)\left(c_{s, 57}+c_{s, 68}\right)+\left(\beta_{51}+\beta_{54}+\beta_{62}+\beta_{63}\right) c_{s, 62} \\
& \left.+\left(\beta_{49}+\beta_{51}+\beta_{62}+\beta_{63}\right) c_{s, 63}+\left(\beta_{66}+\beta_{67}\right)\left(c_{s, 66}+c_{s, 67}\right)+\beta_{55} c_{s, 70}\right) \equiv 0, \\
\equiv & \left(\left(\beta_{45}+\beta_{46}+\beta_{49}+\beta_{52}+\beta_{53}+\beta_{68}+\beta_{69}\right) c_{s, 45}\right. \\
& +\left(\beta_{45}+\beta_{46}+\beta_{47}+\beta_{52}+\beta_{53}+\beta_{62}+\beta_{70}\right) c_{s, 46} \\
& +\left(\beta_{47}+\beta_{49}+\beta_{62}\right) c_{s, 49}+\left(\beta_{50}+\beta_{51}\right)\left(c_{s, 50}+c_{s, 51}\right) \\
& +\left(\beta_{48}+\beta_{52}+\beta_{54}+\beta_{56}+\beta_{67}+\beta_{68}\right) c_{s, 54}+\left(\beta_{53}+\beta_{55}+\beta_{57}+\beta_{68}\right) c_{s, 55} \\
& +\left(\beta_{48}+\beta_{52}+\beta_{54}+\beta_{56}+\beta_{67}\right) c_{s, 56}+\left(\beta_{53}+\beta_{55}+\beta_{57}\right) c_{s, 57} \\
& +\left(\beta_{58}+\beta_{59}\right)\left(c_{s, 58}+c_{s, 59}\right)+\left(\beta_{47}+\beta_{49}+\beta_{62}\right) c_{s, 62} \\
& +\left(\beta_{52}+\beta_{63}+\beta_{64}\right)\left(c_{s, 63}+c_{s, 64}\right)+\left(\beta_{53}+\beta_{65}+\beta_{66}\right)\left(c_{s, 65}+c_{s, 66}\right) \\
& \left.+\left(\beta_{68}+\beta_{69}+\beta_{70}\right) c_{s, 69}+\left(\beta_{69}+\beta_{70}\right) c_{s, 70}\right) \equiv 0 . \\
\hline \sigma_{3}(g)+g &
\end{aligned}
$$


The above equalities show that

$$
\beta_{j}= \begin{cases}\beta_{47} & \text { for } j \in\{48,62, \ldots, 67\} \\ \beta_{58} & \text { for } j \in\{59,60,61\} \\ 0 & \text { otherwise }\end{cases}
$$

The lemma follows.

Now, assume that $[f]$ belongs to the invariant space $\left(\operatorname{Ker}\left[\overline{S q}^{0}\right]_{n_{s}}\right)^{G(4)}$. Since $\Sigma_{4} \subset G(4)$, following Lemmas 3.4.1 and 3.4.2, we have

$$
f \equiv\left(\gamma_{1} q_{s, 1}+\gamma_{2} q_{s, 2}+\gamma_{3} q_{s, 3}+\gamma_{4} q_{s, 4}\right)
$$

wherein $\gamma_{i} \in k$ for all $i$. Direct computing based on the relation $\left(\sigma_{4}(f)+f\right) \equiv 0$, we obtain

$$
\left(\sigma_{4}(f)+f\right) \equiv\left(\left(\gamma_{1}+\gamma_{4}\right) c_{s, 1}+\left(\gamma_{1}+\gamma_{3}\right) c_{s, 3}+\gamma_{1} c_{s, 8}+\left(\gamma_{1}+\gamma_{2}\right) c_{s, 15}+\text { others terms }\right) \equiv 0
$$

This equality implies $\gamma_{i}=0$ for $1 \leqslant i \leqslant 4$. The proof of the theorem is finished.

\subsection{Proof of Theorem 2.2.3}

Let $n_{s, t}:=2^{s+t}+2^{s}-2$. We have seen that the Kameko map

$$
\left[\overline{S q}^{0}\right]_{n_{s, t}}:=\overline{S q}^{0}: Q_{n_{s, t}}^{4} \longrightarrow Q_{2^{s+t-1}+2^{s-1}-3}^{4}
$$

is an epimorphism of $k G(4)$-modules and so

$$
\boldsymbol{Q}_{n_{s, t}}^{4} \cong \operatorname{Ker}\left[\overline{S q}^{0}\right]_{n_{s, t}} \bigoplus Q_{2^{s+t-1}+2^{s-1}-3}^{4}
$$

Using the calculations in Sum [Su14] and Theorem 2.1.8, it follows that, for each $t \geqslant 4$, the coinvariants $k \otimes_{G(4)} P\left(\left(P_{4}\right)_{2^{s+t-1}+2^{s-1}-3}^{*}\right)$ are 1-dimensional if $1 \leqslant s \leqslant 4$ and are 2-dimensional if $s \geqslant 5$. Thus, due to $(25)$, we need to determine $\left(\operatorname{Ker}\left[\overline{S q}^{0}\right]_{n_{s, t}}\right)^{G(4)}$. The below technicality is crucial in the proof of the theorem, but it is quite easy, and so, we state it here with a short sketch only for the reader's convenience.

Lemma 3.5.1. Let $s, t$ be two positive integers such that $t \geqslant 5$. Then, the subspace of $G(4)$ invariants $\left(\operatorname{Ker}\left[\overline{S q}^{0}\right]_{n_{s, t}}\right)^{G(4)}$ is trivial if $s=1,2$ and is 1-dimensional if $s \geqslant 3$.

Outline of the proof. As it is known, from Sum's paper [Su14], if $x$ is an admissible monomial in $\left(P_{4}\right)_{n_{s, t}}$ such that $[x] \in \operatorname{Ker}\left[\overline{S q}^{0}\right]_{n_{s, t}}$, then $\omega_{(s, t)}:=\omega(x)=\underbrace{(2,2, \ldots, 2}_{s \text { times }}, \underbrace{1,1, \ldots, 1}_{t \text { times }})$. The admissible monomial bases of $\left(Q_{n_{s, t}}^{\otimes 4}\right)_{(s, t)}^{2}$ are the sets $\left[\left\{b_{t, 1, j} \mid 1 \leqslant j \leqslant 138\right\} \cup\left\{c_{t, 1, j} \mid 1 \leqslant j \leqslant 7\right\}\right]$ for $s=1$, and $\left[\left\{b_{t, s, j} \mid 1 \leqslant j \leqslant 105\right\}\right]$ for $s \geqslant 2$, wherein the monomials $b_{t, 1, j}, c_{t, 1, j}$ and $b_{t, s, j}$ are given in [Su14]. From these data and our previous results in [Ph21c], by similar calculations as in the proof of Theorem 2.2.1, the lemma follows from the facts that the invariant spaces $\left(\left(\boldsymbol{Q}_{n_{1, t}}^{4}\right)^{\omega_{(1, t)}}\right)^{G(4)}$ and $\left(\left(\boldsymbol{Q}_{n_{2, t}}^{4}\right)^{\omega_{(2, t)}}\right)^{G(4)}$ are trivial and that $\left(\left(\boldsymbol{Q}_{n_{s, t}}^{4}\right)^{\omega_{(s, t)}}\right)^{G(4)}$ is 1-dimensional for arbitrary $s>2$.

Now, for $s=1,2$, let $\rho \in\left(P_{4}\right)_{n_{1, t}}$ and $\bar{\rho} \in\left(P_{4}\right)_{n_{2, t}}$ such that $[\rho] \in\left(\boldsymbol{Q}_{n_{1, t}}^{4}\right)^{G(4)}$ and $[\bar{\rho}] \in$ $\left(\boldsymbol{Q}_{n_{2, t}}^{4}\right)^{G(4)}$, respectively. Since Kameko's homomorphism $\left[\overline{S q}^{0}\right]_{n_{s, t}}: \boldsymbol{Q}_{n_{s, t}}^{4} \longrightarrow \boldsymbol{Q}_{\frac{n_{s, t}}{2}}^{4}$ is an epimorphism of $k G(4)$-modules, $\left[\overline{S q}^{0}\right]_{n_{1, t}}([\rho]) \in\left(\boldsymbol{Q}_{\frac{n_{1, t}}{2}}^{4}\right)^{G(4)}$ and $\left[\overline{S q}^{0}\right]_{n_{2, t}}([\bar{\rho}]) \in\left(\boldsymbol{Q}_{\frac{n_{2, t}}{2}}^{4}\right)^{G(4)}$. Following $\left[\right.$ Su21a], we have $\left(\boldsymbol{Q}_{\frac{n_{1, t}}{2}}^{4}\right)^{G(4)} \subseteq\left\langle\left[p_{4, t}:=\sum_{1 \leqslant j \leqslant 35} d_{t, j}\right]\right\rangle$, and $\left(\boldsymbol{Q}_{\frac{n_{2, t}}{2}}^{4}\right)^{G(4)} \subseteq\left\langle\left[\bar{p}_{4, t}\right]\right\rangle^{2}$, wherein

$$
\bar{p}_{4, t}=\sum_{1 \leqslant \ell \leqslant 3} \sum_{1 \leqslant i_{1} \leqslant \ldots \leqslant i_{\ell} \leqslant 4} x_{i_{1}} x_{i_{2}}^{2} \ldots x_{i_{\ell-1}}^{2^{\ell-2}} x_{i_{\ell}}^{2^{t+1}-2^{\ell-1}}+x_{1} x_{2}^{2} x_{3}^{4} x_{4}^{2^{t+1}-8}
$$

and the elements $d_{t, j}$ are listed as follows: 


$\begin{array}{lll}d_{t, 1}=x_{3}^{2^{t}-1} x_{4}^{2^{t}-1}, & d_{t, 2}=x_{2}^{2^{t}-1} x_{4}^{2^{t}-1}, & d_{t, 3}=x_{2}^{2^{t}-1} x_{3}^{2^{t}-1}, \\ d_{t, 4}=x_{1}^{2^{t}-1} x_{4}^{2^{t}-1}, & d_{t, 5}=x_{1}^{2^{t}-1} x_{3}^{2^{t}-1}, & d_{t, 6}=x_{1}^{2^{t}-1} x_{2}^{2^{t}-1}, \\ d_{t, 7}=x_{2} x_{3}^{2^{t}-2} x_{4}^{2^{t}-1}, & d_{t, 8}=x_{2} x_{3}^{2^{t}-1} x_{4}^{2^{t}-2}, & d_{t, 9}=x_{2}^{2^{t}-1} x_{3} x_{4}^{2^{t}-2}, \\ d_{t, 10}=x_{1} x_{3}^{2^{t}-2} x_{4}^{2^{t}-1}, & d_{t, 11}=x_{1} x_{3}^{2^{t}-1} x_{4}^{2^{t}-2}, & d_{t, 12}=x_{1} x_{2}^{2^{t}-2} x_{4}^{2^{t}-1} \\ d_{t, 13}=x_{1} x_{2}^{2^{t}-2} x_{3}^{2^{t}-1}, & d_{t, 14}=x_{1} x_{2}^{2^{t}-1} x_{4}^{2^{t}-2}, & d_{t, 15}=x_{1} x_{2}^{2^{t}-1} x_{3}^{2^{t}-2} \\ d_{t, 16}=x_{1}^{2^{t}-1} x_{3} x_{4}^{2^{t}-2}, & d_{t, 17}=x_{1}^{2^{t}-1} x_{2} x_{4}^{2^{t}-2}, & d_{t, 18}=x_{1}^{2^{t}-1} x_{2} x_{3}^{2^{t}-2}, \\ d_{t, 19}=x_{2}^{3} x_{3}^{2^{t}-3} x_{4}^{2^{t}-2}, & d_{t, 20}=x_{1}^{3} x_{3}^{2^{t}-3} x_{4}^{2^{t}-2}, & d_{t, 21}=x_{1}^{3} x_{2}^{2^{t}-3} x_{4}^{2^{t}-2}, \\ d_{t, 22}=x_{1}^{3} x_{2}^{2^{t}-3} x_{3}^{2^{t}-2}, & d_{t, 23}=x_{1} x_{2}^{2} x_{3}^{2^{t}-4} x_{4}^{2^{t}-1}, & d_{t, 24}=x_{1} x_{2}^{2} x_{3}^{2^{t}-1} x_{4}^{2^{t}-4} \\ d_{t, 25}=x_{1} x_{2}^{2^{t}-1} x_{3}^{2} x_{4}^{2^{t}-4}, & d_{t, 26}=x_{1}^{2^{2}-1} x_{2} x_{3}^{2} x_{4}^{2^{t}-4}, & d_{t, 27}=x_{1} x_{2} x_{3}^{2^{t}-2} x_{4}^{2^{t}-2}, \\ d_{t, 28}=x_{1} x_{2}^{2^{t}-2} x_{3} x_{4}^{2^{t}-2}, & d_{t, 29}=x_{1}^{3} x_{2}^{5} x_{3}^{2^{t}-6} x_{4}^{2^{t}-4}, & d_{t, 30}=x_{1} x_{2}^{2} x_{3}^{2^{t}-3} x_{4}^{2^{t}-2}, \\ d_{t, 31}=x_{1} x_{2}^{3} x_{3}^{2^{t}-4} x_{4}^{2^{t}-2}, & d_{t, 32}=x_{1} x_{2}^{3} x_{3}^{2^{t}-2} x_{4}^{2^{t}-4}, & d_{t, 33}=x_{1}^{3} x_{2} x_{3}^{2^{t}-4} x_{4}^{2^{t}-2}, \\ d_{t, 34}=x_{1}^{3} x_{2} x_{3}^{2^{t}-2} x_{4}^{2^{t}-4}, & d_{t, 35}=x_{1}^{3} x_{2}^{2^{t}-3} x_{3}^{2} x_{4}^{2^{t}-4} . & \end{array}$

Hence, one obtains $\left[\overline{S q}^{0}\right]_{n_{1, t}}([\rho])=\gamma\left[\varphi\left(p_{4, t}\right)\right]$ and $\left[\overline{S q}^{0}\right]_{n_{2, t}}([\bar{\rho}])=\beta\left[\varphi\left(\bar{p}_{4, t}\right)\right]$ wherein $\gamma, \beta \in k$ and $\varphi$ is the up Kameko map. Because $[\rho] \in\left[\boldsymbol{Q}_{n_{1, t}}^{4}\right]^{G(4)}$ and $[\bar{\rho}] \in\left[\boldsymbol{Q}_{n_{2, t}}^{4}\right]^{G(4)}$, we have $\rho \equiv \gamma \varphi\left(p_{4, t}\right)+\rho^{*}$ and $\bar{\rho} \equiv \beta \varphi\left(\bar{p}_{4, t}\right)+\bar{\rho}^{*}$ wherein $\rho^{*} \in\left(P_{4}\right)_{n_{1, t}}$ and $\bar{\rho}^{*} \in\left(P_{4}\right)_{n_{2, t}}$ such that $\left[\rho^{*}\right] \in \operatorname{Ker}\left[\overline{S q}^{0}\right]_{n_{1, t}}$ and $\left[\bar{\rho}^{*}\right] \in \operatorname{Ker}\left[\overline{S q}^{0}\right]_{n_{2, t}}$, respectively. Using Lemma 3.5.1 and the relations $\sigma_{i}(\rho)+\rho \equiv 0$, and $\sigma_{i}(\bar{\rho})+$ $\bar{\rho} \equiv 0$ for $1 \leqslant i \leqslant 4$, we can obtain, through direct calculations, that $\left(\boldsymbol{Q}_{n_{1, t}}^{4}\right)^{G(4)}$ and $\left(\boldsymbol{Q}_{n_{2, t}}^{4}\right)^{G(4)}$ are respectively generated by $\left[\varphi\left(p_{4, t}\right)\right]$ and $\left[\varphi\left(\bar{p}_{4, t}\right)\right]$. Now, straightforward calculations show that $\zeta_{1, t}:=a_{1}^{(1)} a_{2}^{(1)} a_{3}^{\left(2^{t}-1\right)} a_{4}^{\left(2^{t}-1\right)} \in\left(P_{4}\right)_{n_{1, t}}^{*}$ and $\zeta_{2, t}:=a_{1}^{(1)} a_{2}^{(1)} a_{3}^{(1)} a_{4}^{\left(2^{t+2}-1\right)} \in\left(P_{4}\right)_{n_{2, t}}^{*}$ are $\hat{A}$-annihilated elements. Furthermore, it can be easily seen that $\left\langle\zeta_{1, t}, \varphi\left(p_{4, t}\right)\right\rangle=1$ and $\left\langle\zeta_{2, t}, \varphi\left(\bar{p}_{4, t}\right)\right\rangle=1$, one derives $k \otimes_{G(4)} P_{A}\left(\left(P_{4}\right)_{n_{1, t}}^{*}\right)=\left\langle\left[\zeta_{1, t}\right]\right\rangle$, and $k \otimes_{G(4)} P_{A}\left(\left(P_{4}\right)_{n_{2, t}}^{*}\right)=\left\langle\left[\zeta_{2, t}\right]\right\rangle$. By similar arguments using Theorem 2.1.8 and Lemma 3.5.1, it is not too difficult to verify that

$$
k \otimes_{G(4)} P_{A}\left(\left(P_{4}\right)_{n_{s, t}}^{*}\right)= \begin{cases}\left\langle\left[\zeta_{s, t}\right],\left[\widetilde{\zeta}_{s, t}\right]\right\rangle & \text { if } s=3,4, \\ \left\langle\left[\zeta_{s, t}\right],\left[\widetilde{\zeta}_{s, t}\right],\left[\widehat{\zeta}_{s, t}\right]\right\rangle & \text { if } s \geqslant 5,\end{cases}
$$

wherein the elements

$$
\begin{aligned}
\zeta_{s, t} & :=a_{1}^{(1)} a_{2}^{\left(2^{s}-1\right)} a_{3}^{\left(2^{s+t-1}-1\right)} a_{4}^{\left(2^{s+t-1}-1\right)} \\
\widetilde{\zeta}_{s, t} & :=a_{3}^{\left(2^{s}-1\right)} a_{4}^{\left(2^{s+t}-1\right)} \\
\widehat{\zeta}_{s, t} & :=a_{1}^{(1)} a_{2}^{\left(2^{s-1}-1\right)} a_{3}^{\left(2^{s-1}-1\right)} a_{4}^{\left(2^{s+t}-1\right)}
\end{aligned}
$$

belong to $P_{A}\left(\left(P_{4}\right)_{n_{s, t}}^{*}\right)$. In actual fact, the subsequent steps can be obtained through meticulous hand-written calculations, which we shall omit in the interest of brevity. We leave these calculations to the interested reader for their scrutiny, and thus arrive at the desired conclusion. With this, we have completed the proof of Theorem 2.2.3.

\section{References}

[Ad60] J.F. Adams, On the non-existence of elements of Hopf invariant one, Ann. of Math. (2) 72 (1960), 20-104, https://doi. $\operatorname{org} / 10.2307 / 1970147$.

[Ad52] J. Adem, The iteration of the Steenrod squares in Algebraic Topology, Proc. Natl. Acad. Sci. USA 38 (1952), 720-726, https://doi.org/10.1073/pnas.38.8.720.

[Bo93] J.M. Boardman, Modular representations on the homology of power of real projective space, in Algebraic Topology: Oaxtepec 1991, ed. M. C. Tangor; in Contemp. Math. 146 (1993), 49-70, http://dx.doi.org/10.1090/conm/146.

$\left[\mathrm{BCK}^{+} 66\right]$ A.K. Bousfield, E.B. Curtis, D.M. Kan, D.G. Quillen, D.L. Rector and J.W. Schlesinger, The mod-p lower central series and the Adams spectral sequence, Topology 5 (1966), 331-342, https://doi.org/10.1016/0040-9383(66)90024-3.

[BHH05] R.R. Bruner, L.M. Hà and N.H.V. Hưng, On behavior of the algebraic transfer, Trans. Amer. Math. Soc. 357 (2005), 437-487, https://doi.org/10.1090/S0002-9947-04-03661-X.

[Ch11] T.W. Chen, Determination of $\operatorname{Ext}_{\mathscr{A}}^{5, *}(\mathbb{Z} / 2, \mathbb{Z} / 2)$, Topology. Appl. 158 (2011), 660-689, https://doi.org/10.1016/j.topol. 2011.01 .002 .

[CH11] P.H. Chơn and L.M. Hà, Lambda algebra and the Singer transfer, C. R. Math. Acad. Sci. Paris 349 (2011), 21-23, https://doi.org/10.1016/j.crma.2010.11.008.

[CH12] P.H. Chơn and L.M. Hà, On May spectral sequence and the algebraic transfer, Manuscripta Math. 138 (2012), 141-160, https://doi.org/10.1007/s00229-011-0487-0.

[Fr38] H. Freudenthal, Über die Klassen der Sphärenabbildungen I. Große Dimensionen, Compos. Math. 5 (1938), $299-314$. 
[Ha07] L.M. Hà, Sub-Hopf algebras of the Steenrod algebra and the Singer transfer, Geom. Topol. Monogr. 11 (2007), 101-124, https://doi.org/10.2140/gtm.2007.11.81.

[Hu90] N.H.V. Hưng, The weak conjecture on spherical classes, Math. Z. 231 (1999), 727-743, https://doi.org/10.1007/ PL00004750.

[Hu05] N.H.V. Hưng, The cohomology of the Steenrod algebra and representations of the general linear groups, Trans. Amer. Math. Soc. 357 (2005), 4065-4089, https://doi.org/10.1090/S0002-9947-05-03889-4.

[HQ09] N.H.V. Hưng and V.T.N. Quỳnh, The image of Singer's fourth transfer, C. R. Math. Acad. Sci. Paris 347 (2009), 14151418, https://doi.org/10.1016/j.crma.2009.10.018.

[Ja00] A.S. Janfada, The hit problem for symmetric polynomials over the Steenrod algebra, PhD. thesis, University of Manchester, 2000.

[Ja08] A.S. Janfada, A criterion for a monomial in P(3) to be hit, Math. Proc. Cambridge Philos. Soc. 145, (2008), 587-599, https://doi.org/10.1017/S030500410800162X.

[Ka90] M. Kameko, Products of projective spaces as Steenrod modules, PhD. thesis, The Johns Hopkins University, ProQuest LLC, Ann Arbor, MI, 1990.

[Ka03] M. Kameko, Generators of the cohomology of $B V_{4}$, Preprint (2003), Toyama University, Japan, 8 pages.

[Li08] W.H. Lin, $\operatorname{Ext}_{\mathcal{A}}^{4, *}(\mathbb{Z} / 2, \mathbb{Z} / 2)$ and $\operatorname{Ext}_{\mathcal{A}}^{5, *}(\mathbb{Z} / 2, \mathbb{Z} / 2)$, Topology. Appl. 155 (2008), 459-496, https://doi.org/10.1016/j. topol.2007.11.003.

[Mi95] N. Minami, The Adams spectral sequence and the triple transfer, Amer. J. Math. 117 (1995), 965-985, https://doi.org/ $10.2307 / 2374955$.

[Na04] T.N. Nam, $\mathscr{A}$-générateurs génériques pour l'algèbre polynomiale, Adv. Math. 186 (2004) 334-362, https://doi.org/10. 1016/j.aim.2003.08.004.

[Na08] T.N. Nam, Transfert algébrique et action du groupe linéaire sur les puissances divisées modulo 2, Ann. Inst. Fourier (Grenoble) 58 (2008), 1785-1837, https://doi.org/10.5802/aif.2399.

[Pe87] F.P. Peterson, Generators of $H^{*}\left(\mathbb{R} P^{\infty} \times \mathbb{R} P^{\infty}\right)$ as a module over the Steenrod algebra, Abstracts Papers Presented Am. Math. Soc. 833 (1987), 55-89.

[Ph20] Đ.V. Phúc, The "hit" problem of five variables in the generic degree and its application, Topology. Appl. 282 (2020) 107321, https://doi.org/10.1016/j.topol.2020.107321.

[Ph21a] Đ.V. Phúc, On Peterson's open problem and representations of the general linear groups, J. Korean Math. Soc. 58 (2021), 643-702, https://doi.org/10.4134/JKMS.j200219.

[Ph21b] Đ.V. Phúc, On the dimension of $H^{*}\left(\left(\mathbb{Z}_{2}\right)^{\times t}, \mathbb{Z}_{2}\right)$ as a module over Steenrod ring, Topology. Appl. 303 (2021), 107856, https://doi.org/10.1016/j.topol.2021.107856.

[Ph21c] Đ.V. Phúc, A note on the modular representation on the $\mathbb{Z} / 2$-homology groups of the fourth power of real projective space and its application, Preprint (2021), available online at https://www.researchgate.net/publication/353065445.

[Ph22a] Đ.V. Phúc, On the hit problem for the polynomial algebra and the algebraic transfer, Preprint (2022), 40 pages, available online at https://www.researchgate.net/publication/364695637.

[Ph22b] Đ.V. Phúc, On modules over the mod 2 Steenrod algebra and hit problems, Preprint (2022), 44 pages, available online at https://www.researchgate.net/publication/365597765, submitted for publication.

[Ph23a] Đ.V. Phúc, A note on the hit problem for the polynomial algebra of six variables and the sixth algebraic transfer, J. Algebra 613 (2023), 1-31, https://doi.org/10.1016/j.jalgebra.2022.08.028.

[Ph23b] Đ.V. Phúc and S. Azizi, On the (symmetric) hit problems for the (symmetric) polynomial algebras and the algebraic transfer, Preprint (2023), 40 pages, submitted for publication.

[Pr70] S.B. Priddy, Koszul resolutions, Trans. Amer. Math. Soc. 152 (1970), 39-60, https://doi.org/10.1090/ S0002-9947-1970-0265437-8.

[Si89] W.M. Singer, The transfer in homological algebra, Math. Z. 202 (1989), 493-523, https://doi.org/10.1007/BF01221587.

[Si91] W.M. Singer, On the action of Steenrod squares on polynomial algebras, Proc. Amer. Math. Soc. 111 (1991), 577-583, https://doi.org/10.2307/2048351.

[Su10] N. Sum, The negative answer to Kameko's conjecture on the hit problem, Adv. Math. 225 (2010), 2365-2390, https: //doi.org/10.1016/j.aim.2010.04.026.

[Su14] N. Sum, The hit problem for the polynomial algebra of four variables, Preprint (2014), 240 pages, arXiv:1412.1709.

[Su15] N. Sum, On the Peterson hit problem, Adv. Math. 274 (2015), 432-489, https://doi.org/10.1016/j.aim.2015.01.010.

[Su18] N. Sum, On the determination of the Singer transfer, Vietnam J. Sci., Technol. Eng. 60 (2018), 3-16, https://doi.org/10. 31276/VJSTE.60(1).03.

[Su21a] N. Sum, On the determination of the Singer transfer, Preprint (2021), 17 pages, arXiv:1710.07895.

[Su21b] N. Sum, The squaring operation and the Singer algebraic transfer, Vietnam J. Math. 49 (2021), 1079-1096, https: //doi.org/10.1007/s10013-020-00423-1.

[Su23] N. Sum, The squaring operation and the hit problem for the polynomial algebra in a type of generic degree, J. Algebra 622 (2023), 165-196, https://doi.org/10.1016/j.jalgebra.2023.01.018.

[Th54] R. Thom, Quelques propriétés globales des variétés differentiables, Comment. Math. Helv. 28 (1954), 17-86, https://doi. org/10.1007/BF02566923.

[WW18a] G. Walker and R.M.W. Wood, Polynomials and the mod 2 Steenrod Algebra. Volume 1: The Peterson hit problem, in London Math. Soc. Lecture Note Ser., Cambridge Univ. Press, 2018.

[WW18b] G. Walker and R.M.W. Wood, Polynomials and the mod 2 Steenrod Algebra. Volume 2: Representations of GL $\left(n, \mathbb{F}_{2}\right)$, in London Math. Soc. Lecture Note Ser., Cambridge Univ. Press, 2018..

[Wa60] C.T.C. Wall, Generators and relations for the Steenrod algebra, Ann. of Math. (2) 72 (1960), 429-444, http://doi.org/ $10.2307 / 1970225$.

[Wa67] J.S.P. Wang, On the cohomology of the mod-2 Steenrod algebra and the non-existence of elements of Hopf invariant one, Illinois J. Math. 11 (1967), 480-490, http://doi.org/10.1215/ijm/1256054570.

[Wo89] R.M.W. Wood, Steenrod squares of polynomials and the Peterson conjecture, Math. Proc. Cambriges Phil. Soc. 105 (1989), 307-309, https://doi.org/10.1017/S0305004100067797. 